\title{
Quantitative Analysis of Ice Accretion Roughness \\ Using Spectral and Stochastic Techniques
}

\author{
by \\ D. J. Orr Jr.
}

B.S.E. Aerospace Engineering

Arizona State University, 1991

B.S. Mathematics

Arizona State University, 1993

Submitted to the Department of Aeronautics and Astronautics

in partial fulfillment of the requirements for the degree of

Master of Science in Aeronautics and Astronautics

at the

MASSACHUSETTS INSTITUTE OF TECHNOLOGY

June 1996

(c) Massachusetts Institute of Technology 1996. All rights reserved.

Author .

Department of Aeronautics and Astronautics

May 23, 1996

Certified by .................

Kenneth S. Breuer

Assistant Professor

Thesis Supervisor

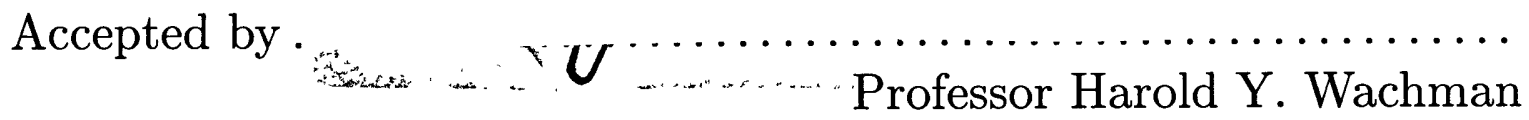

Chairman, Department Graduate Committee

ingsadustrinsmun

OF TECHWHOLOY

JUN 111996

Aero 


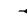




\title{
Quantitative Analysis of Ice Accretion Roughness Using Spectral and Stochastic Techniques
}

\author{
by \\ D. J. Orr Jr. \\ Submitted to the Department of Aeronautics and Astronautics \\ on May 16, 1996, in partial fulfillment of the \\ requirements for the degree of \\ Master of Science in Aeronautics and Astronautics
}

\begin{abstract}
A self-consistent scheme for relating wind tunnel ice accretion roughness to the resulting enhancement of heat transfer is described. Two methods for quantitative analysis of early ice accretion roughness are presented and discussed. The first technique employs a "structural-stochastic" Markov Random Field (MRF) model to simulate realistic-looking synthetic accretion images. The MRF technique can also be used to extract parameters from existing images although the physical interpretation of the parameters is difficult. The second technique uses a spectral estimation technique (SET) which extracts physically descriptive parameters by comparing scan lines from the experimentally-obtained accretion images to a prescribed test function. This technique is shown to yield consistent and physically relevant results when applied to a series of images from the NASA Lewis Icing Research Tunnel (IRT). An experimental technique is then presented for constructing physical roughness models suitable for wind tunnel testing that match the SET parameters extracted from the IRT images. The icing castings and modeled roughness are tested for enhancement of boundary layer heat transfer using infrared techniques in a "dry" wind tunnel.
\end{abstract}

Thesis Supervisor: Kenneth S. Breuer

Title: Assistant Professor 



\section{Acknowledgments}

First and foremost, I'd like to thank my personal Lord and Savior, Jesus Christ, to whom I owe everything. Secondly, I'm forever indebted to my family - Mom, Dad, Hope, Althea, and Rustelle - this thesis is a product of your investment in me and your agape love over the years. You represent my past, present, and future. Mom and Dad, "acknowledgment" is a gross understatement for what I owe you; you have my deepest appreciation. Althea and David, thanks for being good friends, no trip back to the Valley of the Sun would be complete without a visit to see you and the kids. Hope, from the earliest days of the "Grit Bailout," you've always been there for me. Thank you. Grandma, June, Gramsie, Auntie Susie, Uncle Jake (I'm sorry I wasn't there to say good-bye), Keith, the rest of the Kellers, Larry and Kathleen, thanks for being family.

I'm also very grateful for my "family away from home" - Rev. Dr. Samuel and Genevieve Bombara and the rest of the family at St. Paul Evangelical Church. You've all helped me keep the proper perspective on life at a time when it's easy to get priorities out of line. Thanks.

Special thanks to my advisors over the years, Phil Jankowski, Valana Wells, Bill Saric, John Hansman and Kenny Breuer.

I've also had the benefit of many good friends over the years to whom I'm very grateful. For those from the early days, at the risk of omitting good friends, I'd like to thank Dave B., Joe M., Joey K., Alan A., Boyd E., Todd G., Troy E., Ted B., Jeff D., Mike R., Allen P., Mary R., Susan B., and Sherry K. And of course, Sheila W. As for my fellow midgets, Troy G., Troy W., Brian V., Kent B., Ryan P., Wade W., Dan P., Nadine M., Susie R., thanks. More recently, Stephen P., thanks for the ice tea in the desert. To Tom and Lisa Warner - enjoy the American Dream, you've earned it. Shiela, you were a fixture in my undergraduate career, gracias. I'm also very grateful to the entire ASUUWT team for all I learned from them. Thanks, Bill Tierney, you added another dimension to my undergraduate education. I owe a lot to my good friend and roommate, Ed Piekos. Thanks, I've learned much from you. Ruben, thanks for being a good friend and for all the help you've given me around the lab.

Many valuable lessons on sacrifice, goal-setting, and the value of a good work ethic came from coaches. Thanks Coach Krikac, Mr. Smith, Coach Johnson, Coach Glasser, Coach Blake, Klinger, and Coach Pond.

My success as a student certainly required some influential teachers. Mrs. Holm, sorry for all the aggravation I caused you. But, hey, it would have taken all the fun out of it if you had known that I'd eventually get serious about school! Mr. Brink, tractors just aren't for me, but I think you knew that, because I still apply the skills you helped me develop. Mrs. Burkett, thanks for not giving up on me. Connie, if it hadn't been for you, I wouldn't be writing this. Thanks.

Believe it or not, I have had "real" jobs. To Jimmy Otto I owe thanks for my first job. Thanks Cecil, I hope you're happy where ever you are. Extra special thanks to Bill Fournier and the crew at AZP10. 

"'You're a writer?' asked the poet with interest. The guest's face darkened, and he shook his fist at Ivan and then said, 'I am the Master.' "

- Mikhail Bulgakov's The Master and Margarita 



\section{Contents}

1 Introduction $\quad 13$

1.1 Motivation . . . . . . . . . . . . . . . . 13

1.2 Icing Experiments . . . . . . . . . . . . . . . 13

1.2 .1 Types of Ice Accretions . . . . . . . . . . . . . . . . 14

1.3 Coupling of Roughness and Heat Transfer . . . . . . . . . . . 14

1.3.1 Multi-Zone Ice Accretions . . . . . . . . . . . . . . . 15

1.3 .2 Horn Growth . . . . . . . . . . . . . . . . . 15

1.3.3 Previous Roughness Studies . . . . . . . . . . . . 16

1.3.4 Previous Heat Transfer Studies . . . . . . . . . . . . . 16

1.4 Current Ice Accretion Modeling . . . . . . . . . . . . . . 18

1.4.1 The Glaze Ice Freezing Fraction Problem . . . . . . . . . . 18

1.4 .2 The Role of ESGR . . . . . . . . . . . . . . . . 18

1.5 Overview of the Current Study . . . . . . . . . . . . . . . 19

1.5.1 Replacement of the ESGR Heat Transfer Model . . . . . . . 19

1.5.2 Completing the New Approach . . . . . . . . . . . . 19

1.5 .3 Outline ................... . 20

2 Sample Images 23

2.1 NASA Lewis IRT Video Data . . . . . . . . . . . . . . 23

2.2 Icing Roughness Castings . . . . . . . . . . . . . . . . . . 24

3 Markov Random Field Texture Analysis 27

3.1 Informal MRF Overview . . . . . . . . . . . . . . . 27

3.2 Introduction to Stochastic Texture Modeling . . . . . . . . . . 28

3.3 MRF Framework for Image Generation . . . . . . . . . . . . . . . 29

3.3.1 Random Variable Selection . . . . . . . . . . . . . . 30

3.3.2 Besag Codings and Classification of Markov Random Fields . 30

3.3.3 MRF Simulation: The Method of Rejection/Selection . . . . 32

3.3.4 Practical MRF Simulation . . . . . . . . . . . . . . . . 33

3.3.5 Estimation of Clustering Parameters . . . . . . . . . . . 34

3.4 MRF Analysis of Accretion Images . . . . . . . . . . . . . 34

3.5 Discussion . . . . . . . . . . . . . . . . . . 35

4 Spectral Techniques for Texture Analysis 37

4.1 Spectral Description of Roughness . . . . . . . . . . . . . 37 
4.2 Spectral Estimation Technique . . . . . . . . . . . . . . . 38

4.2 .1 Basis . . . . . . . . . . . . . . . . . . 38

4.2 .2 Algorithm . . . . . . . . . . . . . . . . . . . . . . 39

4.2 .3 SET Refinements . . . . . . . . . . . . . . . . . . 41

4.2.4 Spectral Implications of Test Function Parameters . . . . . . . 42

4.3 Validation of the SET . . . . . . . . . . . . . . . 43

4.3 .1 Rectangular Bead Arrays . . . . . . . . . . . . . . . . 43

4.3 .2 Pseudo-random Bead Arrays . . . . . . . . . . . . . . . . . 44

4.4 Application of SET to Experimental Data . . . . . . . . . 45

4.4 .1 IRT Close-Up Video Data . . . . . . . . . . . . . . . . 45

4.4 .2 IRT Roughness Castings . . . . . . . . . . . . . . . . . 45

4.5 Discussion . . . . . . . . . . . . . . . . . . 46

5 Simulated Roughness for Dry Wind Tunnel Testing 49

5.1 Use of the SET for Roughness Construction . . . . . . . . . . . 49

5.2 Hardware . . . . . . . . . . . . . . . . . . 49

5.3 Syringe Calibration with the SET . . . . . . . . . . . 51

5.4 Modeling of Casting Data . . . . . . . . . . . . . . 51

5.5 Wind Tunnel Testing . . . . . . . . . . . . . . . 53

6 Conclusions $\quad 55$

A Experimental Data and SET Diagrams $\quad 57$

B Validation of the MRF Code $\quad 69$

C Derivations for Markov Random Field Analysis $\quad 75$

C.1 The Method of Rejection/Selection . . . . . . . . . . . . 75

C.2 Besag's Lattice Probability Quotient . . . . . . . . . . . . 76 


\section{List of Figures}

1-1 Schematic of current and proposed heat transfer model in LEWICE.

2-1 Schematic of IRT setup. Close-up images analyzed in the current study are acquired from the close-up window camera. Figure reproduced from Hansman et al. [5] . . . . . . . . . . . . . . .

2-2 Time sequence of glaze accretion. Dark regions in upper portion of images are from markings on the wind tunnel model and were cropped out of data used for streamwise calculations. Field of view is approx. $1 \mathrm{~cm} \times 1 \mathrm{~cm}$. Cloud conditions: $T=25^{\circ} \mathrm{F}, U=150 \mathrm{mph}, \mathrm{LWC}=$ $1.0 \mathrm{~g} / \mathrm{m}^{3}, M V D=20 \mu \mathrm{m} \ldots \ldots \ldots$

2-3 Time sequence of mixed accretion. Dark regions in upper portion of images are from markings on the wind tunnel model and were cropped out of data used for streamwise calculations. Field of view is approximately $1 \mathrm{~cm} \times 1 \mathrm{~cm}$. Cloud conditions: $T=25^{\circ} \mathrm{F}, U=100 \mathrm{mph}, L W C=$ $0.7 \mathrm{~g} / \mathrm{m}^{3}, M V D=15 \mu \mathrm{m} \ldots \ldots \ldots$

2-4 Surface roughness casting image from a model used in the heat transfer experiments of Masiulaniec et al.[8] Scale on the image is in $\mathrm{mm}$. .

3-1 Besag codings: neighbors of the point $x \ldots \ldots \ldots$. . . . . . . . . . . . .

3-2 MRF Simulation of Glaze Ice Accretion . . . . . . . . . . . . . . . . 36

4-1 (a) Original image. (b) Filtered image with $2 \%$ of modes retained. . . 38

4-2 Sample test function for $D=12, P=51 \ldots \ldots$. . . . . . . . . 39

4-3 Comparison of experimental data and the corresponding optimal test function. . . . . . . . . . . . . . . .

4-4 Effects of $P$ and $D$ on test function optimization. (upper left) optimal; (upper right) $D$ too small; (lower left) $D$ too large; (lower right) $P$ too small. $L$ is the inter-bead spacing: $L=P-D$. . . . . . . . . . . . 42

4-5 Rectangular Bead Array Test Case . . . . . . . . . . . . . . 43

4-6 Pseudo-Random Bead Array Test Case . . . . . . . . . . . . . . . . . 44

4-7 SET Histograms for Mixed Accretion . . . . . . . . . . . . . . . 46

4-8 SET Histograms for Icing Casting . . . . . . . . . . . . . . . . 47

5-1 Three-dimensional traverse for automated epoxy bead deposition. X, $\mathrm{Y}$ and $\mathrm{Z}$ axes control syringe position while $\mathrm{W}$ axis controls the syringe

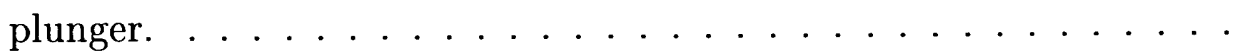


5-2 Three-dimensional traverse for automated epoxy bead deposition. The $\mathrm{W}$ axis and syringe are clearly visible as well as the substrate and epoxy beads. . . . . . . . . . . . . . . .

5-3 Epoxy bead array for SET-based calibration of syringe. Horizontal field of view is $7 \mathrm{~cm}$. Various syringe plunger displacements are used to produce beads of different sizes $(0.4 \mathrm{~mm}-1.4 \mathrm{~mm})$. . . . . . . . . 52

5-4 SET-based calibration curve for syringe/epoxy assembly. . . . . . . 52

5-5 Experimental Epoxy Bead Arrays $(17 \mathrm{~mm} \times 28 \mathrm{~mm}) \quad \ldots . . . . .53$

A-1 Grayscale images for mixed accretion T184.19.52 . . . . . . . . . . 58

A-2 SET distribution for mixed accretion T184.19.52 . . . . . . . . . . 59

A-3 Grayscale images for glaze accretion T184.22.05 . . . . . . . . . . . 60

A-4 SET distribution for glaze accretion T184.22.05. . . . . . . . . . . 61

A-5 Grayscale images for mixed accretion T184.20.26. . . . . . . . . . 62

A-6 SET distribution for mixed accretion T184.20.26. . . . . . . . . . . 63

A-7 Grayscale images for mixed accretion $\mathrm{T} 184.22 .34 \ldots \ldots . . . . . .64$

A-8 SET distribution for mixed accretion T184.22.34. . . . . . . . . . . 65

A-9 Grayscale images for glaze accretion T184.21.32 . . . . . . . . . . 66

A-10 SET distribution for glaze accretion T184.21.32 . . . . . . . . . . 67

B-1 MRF Textures of Cross and Jain [2] . . . . . . . . . . . . 70

B-2 Typical behavior of number of pixel exchanges for code of Cross and Jain [2] . 1 iteration $=256$ attempted exchanges. . . . . . . . . . . . . 71

B-3 MRF Texture Simulation . . . . . . . . . . . . . . . 72

B-4 Behavior of number of pixel exchanges for MRF code of the current study. . . . . . . . . . . . . . . . . 73

B-5 4 - bit, $4^{\text {th }}$-order, Gray-scale MRF Image . . . . . . . . . . 73 


\section{Chapter 1}

\section{Introduction}

\subsection{Motivation}

Aviation accidents believed to be related to icing conditions underscore the need to develop further insight into the physics of ice accretion on aircraft. Research efforts utilizing icing wind tunnels, flight testing, and computational models have provided a valuable base of knowledge. For a given aircraft geometry, the characteristics of an ice accretion are known to depend upon factors such as airspeed, temperature, mass of water per unit volume (Liquid Water Content or LWC), and the size of the water droplets in the flow (Mean Volume Diameter or MVD). As super-cooled water droplets impinge upon the aircraft surface, the percentage of each droplet that freezes, the freezing fraction, depends upon how effectively the latent heat of fusion of the water can be removed [5]. Thus, ice formation is dependent upon heat transfer at the substrate.

Both experimental and computational investigative approaches have their own deficiencies. Icing wind tunnel tests are expensive and securing run-time at the few available facilities can be quite competitive. Flight testing, in addition to being expensive, also poses hazards for the flight crews involved. Although successful in some icing regimes, computational models sometimes lack strong correlation with the real-world accretions to be modeled. However, supported by practical knowledge from icing experiments, improvements in computational modeling hold the potential for substantial savings in the overhead associated with experimental efforts.

\section{$1.2 \quad$ Icing Experiments}

Experiments in icing wind tunnels have played a key role in understanding the physics of ice accretion on aerodynamic bodies. Early work in this area included the icing experiments of Olsen and Walker[11] at the NASA Lewis Icing Research Tunnel (IRT) which produced close-up film images of accretions near the stagnation region on the icing body. More recent IRT experiments[5] have provided high magnification, grayscale video images which represent a continuous record of developing ice accretions that spans from the first seconds of ice formation until the long term ice shape has 
been established approximately 10 minutes later. Other data available from the IRT experiments include overview video images and photographs of long-term global ice shapes, direct tracings of ice accretion shapes taken in model cross-sectional planes, and close-up digital camera images of icing roughness[5]. In addition, Masiulaniec et al.[8] developed a technique for making investment castings of actual ice shapes from IRT experiments.

\subsubsection{Types of Ice Accretions}

IRT experiments have shown that ice accretions may be divided into three categories: rime, glaze, and mixed. Rime accretions are associated with low LWC and low temperatures [5]. The key feature of rime accretions is that all of the incoming water freezes upon impact, resulting in a freezing fraction of unity. Rime ice is characterized by a milky white color and low density which are a consequence of air bubbles trapped between frozen droplets [5]. At sites on the substrate where surface roughness is located, the increased efficiency in collecting water droplets from the flow may result in localized accelerations in the rate of ice growth. This forms a small protuberance, known as a feather, that grows toward the direction of the impinging water droplets[5].

Glaze accretions, occurring at relatively high LWC and high temperature [5], are typically of high density and have a transparent appearance due to the absence of the trapped air bubbles present in the rime case [5]. Under glaze conditions, there is insufficient heat transfer to remove all of the latent heat of fusion of the incoming water and freeze-out of the droplets is incomplete [21] so that a freezing fraction of less than unity results. Water which does not freeze runs downstream on the substrate as surface water in the form of a thin film or as rivulets.

As the term suggests, mixed accretions result from combinations of rime and glaze ice [5]. Mixed ice is somewhat of a borderline case between glaze and rime ice since cloud conditions will support wet icing in the stagnation region of the icing body and dry icing in adjacent off-stagnation regions. Consequently, local freezing fractions in a given accretion may vary from a value of 1.0 (rime ice) to lower values observed for glaze ice.

\subsection{Coupling of Roughness and Heat Transfer}

The initial roughness in an ice accretion establishes the convective heat-transfer coefficient and subsequently determines the freeze-out of excess surface water under glaze conditions[20]. Consequently, early stage roughness controls the rate of formation of more ice. In addition, Kerho and Bragg[6] showed that distributed surface roughness near the stagnation region of an airfoil such as that due to a glaze ice accretion also plays an important role in triggering the laminar-turbulent transition of the boundary layer. It was also discovered that the transition location of the boundary layer did not occur immediately at the roughness element as assumed in the classical work of Nikuradse[10], but instead, at some location downstream. This is not surprising 
since the favorable pressure gradient associated with the upper side of the airfoil should delay transition while the work of Nikuradse[10] was conducted in a constant pressure gradient. Since it is well known that transition to turbulence results in a substantial increase in convective heat transfer, one might expect more ice growth at off-stagnation regions on an icing body at the transition location[5].

\subsubsection{Multi-Zone Ice Accretions}

This coupling of roughness and heat transfer is so central to the icing problem that one can explain many different icing phenomena in terms of the enhancement of convective heat transfer by surface roughness. For example, in the ice accretion films of Olsen and Walker[11], it was discovered that there was a distinct boundary between a "smooth ice" zone near the stagnation line of the icing body and a downstream "rough ice" zone. The location of the smooth-rough boundary was observed to move toward the stagnation region with increasing time. Yamaguchi et al.[21] explain this phenomenon in terms of the enhancement of convective heat transfer due to laminarturbulent boundary layer transition. When there is insufficient heat transfer to freeze surface water near the stagnation region of the icing body, water runback occurs in the form of a thin film. For a sufficiently high local Reynolds number, the boundary layer transitions to turbulence at some location downstream of the water film. When the runback water reaches the region of enhanced heat transfer associated with the turbulent boundary layer, the latent heat of fusion of the water is removed and ice is formed. This newly formed icing roughness enhances the local convective heat transfer so that more runback water is frozen and a smooth-rough boundary is established in the accretion. Eventually, additional freeze-out of surface water on the windward side of the roughness boundary causes the rough ice region to creep upstream toward the stagnation region, as experimentally observed by Olsen and Walker[11].

\subsubsection{Horn Growth}

Because the freezing fraction is unity everywhere in a rime accretion, the macroscopic ice shape that emerges at long time scales of the accretion is relatively uniform and aerodynamically shaped[5]. However, under certain conditions, the effect of surface water runback can cause large protuberances known as "horns." For mixed ice conditions, especially those with higher MVD and/or higher LWC, feather-like formations may be initiated near the smooth-rough boundary where higher heat transfer occurs. Because these protuberances have a freezing fraction of 1.0 and depend upon the direct collection of water from the freestream, they grow into the direction of the oncoming flow. Such growths are called "type A" horns[5]. At warmer temperatures, the freezing fraction in the off-stagnation rough ice zone may be less than 1.0 so that runback water feeds the growth of any roughness elements rather than direct capture of moisture from the freestream. Therefore, near large protuberances where enhancement of heat transfer is high, ice growth will be normal to the substrate forming what are known as "type B" horns[5]. 


\subsubsection{Previous Roughness Studies}

By studying the flow of water through pipes, Nikuradse[10] summarized the effect of surface sand grain roughness on exit velocity profile, head loss, and mass flow rate in terms of a "roughness" Reynolds number:

$$
R e_{k}=\frac{u_{\tau} k}{\nu}
$$

where $\nu$ is the kinematic viscosity of the fluid and $k$ is the roughness height of the sand grains. $u_{\tau}$ is the so-called "friction velocity" given by:

$$
u_{\tau}=\sqrt{\frac{\tau_{w}}{\rho}}
$$

where $\tau_{w}$ is the wall shear stress and $\rho$ is the fluid density.

Note that $R e_{k}$ is simply the roughness height cast in terms of the familiar nondimensional wall units of turbulent boundary layer analysis, $y^{+}$. Nikuradse discovered three regimes delineated by critical values of $R e_{k}$. For roughness elements that are buried in the viscous sublayer of the boundary layer $\left(R e_{k}<5\right)$, the surface is classified as hydraulically smooth and skin friction depends only on the Reynolds number of the flow. As roughness elements begin to protrude through the viscous sublayer $(5 \leq$ $R e_{k}<70$ ), the so-called transitionally rough regime is entered in which skin friction was observed to depend upon both roughness height and the Reynolds number of the overall flow. Finally, in the "fully rough" regime $\left(70 \leq R e_{k}\right)$, roughness elements are large enough to eliminate the viscous sublayer, and the skin friction becomes independent of Reynolds number and depends only upon sand grain height.

Schlichting[17] extended the concept of Nikuradse's sand grain roughness to roughness composed of well-defined, primitive objects. Specifically, the flow of water over arrays of spheres, spherical segments, angle sections, and cones was studied. When the exit velocity distributions were plotted with the wall-normal coordinate normalized by the roughness height and the velocity normalized by the rough wall skin friction velocity, it was found that the profiles were similar for different freestream speeds and were effected only by roughness height and spacing. That is, the details of the individual roughness elements of a fixed height and spacing were unimportant from the standpoint of mean velocity profiles. This led to the notion of "equivalent sand grain roughness" (ESGR) since the key parameters were essentially Nikuradse's sand grain parameter, $k$, and the roughness element density.

\subsubsection{Previous Heat Transfer Studies}

Smith and Epstein[18] extended the concept of sand grain roughness to not only correlate head loss of steam in pipes, but also to correlate the enhancement of convective heat transfer due to sand grain roughness. For a given roughness height, $k$, one could plot Reynolds number vs. heat transfer coefficient. Using a smooth-walled pipe as a baseline, it was shown that increasing the size of the roughness in sand-roughened 
pipes increases the convective heat transfer at the pipe wall.

The work of Henry et al.[4] utilizes infrared (IR) thermography to measure the enhanced heat transfer on roughness samples in dry wind tunnel experiments. The experiments used an IR camera to record changes in temperature distributions produced by IR heating of hemispherical roughness elements in flat plate boundary layers for both laminar and turbulent cases. A steady-state energy balance analysis of the boundary layer flow was used to obtain an expression for the local heat transfer coefficient in a region of the flow perturbed by roughness normalized by the heat transfer coefficient from an unperturbed region:

$$
\frac{h_{p}}{h_{u}}=\frac{T_{s u r_{u}}-T_{o}}{T_{s u r_{p}}-T_{o}}
$$

where $h_{p}$ is the convective heat transfer coefficient from the perturbed region, $h_{u}$ is the convective heat transfer coefficient from the unperturbed region, $T_{s u r_{u}}$ is the surface temperature of the unperturbed region, $T_{s u r_{p}}$ is the surface temperature of the perturbed region and $T_{o}$ is the freestream temperature. Thus, measurement of temperatures is sufficient to gauge heat transfer enhancement due to the roughness in the boundary layer.

The authors found that there was a threshold roughness element height to boundary layer thickness ratio below which no significant enhancement of heat transfer occurred. Laminar boundary layers produced increases in the convective heat transfer coefficient that were up to three times as great as those observed in turbulent boundary layers. This was partly due to the fact that the turbulent flow produced a global enhancement of heat transfer so that gains in $h_{p}$ were partly offset by increases in $h_{u}$. The spacing between the roughness elements was also found to be important. Heat transfer enhancement on individual beads was apparent at larger spacings while densely packed roughness arrays produced a constant level of enhancement across the entire array. This constant level of enhancement was observed to increase with Reynolds number.

Masiulaniec et al.[8] conducted measurements of heat transfer on investment casting tiles of IRT ice shapes from flat plate models. Instead of heating the substrate from the "flow side" of the model with IR heaters as in the work of Henry et al.[4], this study applied heat to the back side of roughness tiles exposed to various flow conditions. Each tile represented a sector of a larger investment casting of icing roughness and was thermally insulated and packed into an array with the adjacent tiles from the same original casting. Convective heat transfer for each tile was measured by monitoring the amount of heat addition necessary to keep each tile at constant temperature. Since the tiles had dimensions of $17 \mathrm{~mm} \times 28 \mathrm{~mm}$, this technique effectively averaged the heat transfer over a relatively large area compared to the sub-millimeter scales achieved by the IR technique of Henry et al.[4]. Since good resolution of the heat transfer field is helpful for understanding evolution of the localized ice growths that dominate the micro-physics of icing, the IR techniques may provide more flexibility for describing variations in heat transfer than those of Masiulaniec et al[8]. 


\subsection{Current Ice Accretion Modeling}

\subsubsection{The Glaze Ice Freezing Fraction Problem}

As discussed above, roughness gives rise to the enhancement of heat transfer which subsequently determines the local freezing fractions in the accretion. In turn, the local freezing fraction quantifies the distinguishing aspect of glaze accretions, surface water. Herein lies a key obstacle in developing an effective computational model for aircraft icing: How much water should remain as ice and how much should be transported downstream as liquid? The answer depends on the impact of surface roughness upon heat transfer. In the case of rime ice, the solution is simple: the modeler need not perform any bookkeeping on what mass of water freezes and what mass of water runs back since all droplets "stick" where they strike the substrate. In fact, ballistic computational models have produced simulated accretions that have a striking resemblance to experimentally observed ice shapes [13]. Determining local freezing fractions in glaze cases is much more difficult. The failure of current stateof-the-art icing codes to effectively address this issue is evident in their inability to produce realistic glaze ice shapes[20]. Since glaze ice is present in mixed ice, current models are no more effective in producing mixed ice shapes than they are in producing glaze ice shapes. In light of these observations, it is clear that an icing model that is to be effective in the glaze regime must incorporate a realistic heat transfer model based upon quantitative characterization of early-stage surface roughness so that local freezing fractions can be adequately simulated.

\subsubsection{The Role of ESGR}

Ice accretion codes such as NASA's LEWICE represent the enhanced heat transfer that results from surface roughness[20] with the Equivalent Sand Grain Roughness (ESGR) model developed by Schlichting[17] using the work of Nikuradse[10] as a guide. From the standpoint of roughness modeling, ESGR is used to reduce the substantial complexity of early glaze accretions to a single parameter, $k$, upon which heat transfer correlations are based. ESGR has two primary roles in LEWICE[20]. Since LEWICE assumes that the flow over the icing body is initially laminar, a boundary layer transition location must be determined. This is accomplished with a simple switch based on ESGR; if $R e_{k}$ exceeds some critical value, the flow is assumed to transition to turbulence at that point. Roughness is assumed to have no effect upon laminar heat transfer unless the protuberances extend beyond the boundary layer. Secondly, once the flow is turbulent, $k$ is used as an input parameter to a set of turbulent heat transfer equations. Due to the complexity of these equations, it is difficult to determine the dependence of turbulent heat transfer on $k$ using analytical tools. However, the sensitivity of LEWICE's turbulent heat transfer coefficient on variations in $k$ was demonstrated with an empirical study by Yamaguchi et al.[20]

The limitations of the ESGR correlations are particularly evident when one recalls that their original definition was only to establish a uniform standard for correlating the pressure drop along a pipe with a rough surface[10] and had no relation to heat 
transfer, let alone multi-phase ice accretions. ESGR is often used in current icing codes as a free parameter that is adjusted until the simulated ice shape matches a given experimentally observed ice shape. It is apparent that this description of roughness is problematic when one realizes that the value of $k$ required to create realistic ice shapes often bears no relation to the roughness scales observed in the real accretion. Consequently, a priori determination of glaze ice shapes is difficult.

\subsection{Overview of the Current Study}

\subsubsection{Replacement of the ESGR Heat Transfer Model}

The shortcomings of the ESGR heat transfer and transition model used in LEWICE become starkly apparent when one considers the current role of ESGR in the overall scheme of computer-based ice shape prediction. Use of the ESGR parameter $k$ as an input quantity to LEWICE is depicted as the "Equivalent Sand Grain Roughness" module on the left side of Figure 1-1. In this schematic, it is clear that much of the available icing data produced by IRT experiments is excluded from the ESGR model. This data base is represented as the "Early Accretion IRT Data" module on the right side of Figure 1-1. Currently, the full potential of this information is not realized by LEWICE since ESGR does not quantitatively connect the experimental icing images to the predicted ice shapes.

Similarly, the insights into the effect of roughness on enhancement of convective heat transfer provided by the experiments of Henry et al.[4] are also absent from the ESGR model. This information is denoted on the right side of Figure 1-1 as the " 'Dry' Heat Transfer Experiments" module.

The goal of this work is to provide an alternative to the ESGR heat transfer correlations based upon interpretation of close-up images detailing ice accretion microphysics and dry heat transfer tests on simulated icing roughness. In other words, it is necessary to develop the modules entitled "Extract Roughness Characteristics" and "Construct Physical Approximations" shown as part of the "Proposed Approach" on the right side of Figure 1-1.

\subsubsection{Completing the New Approach}

From Figure 1-1 it is clear that in order to incorporate the experimentally obtained icing images into an improved heat transfer model, one must have some method of objectively interpreting the data. This missing link in the scheme is depicted by the "Extract Roughness Characteristics" module. Quantitative analysis of such data requires techniques which fall under the rubric of image processing. Since the objective of the current study is to use image processing on experimental data to improve efforts to assess the impact of roughness on heat transfer enhancement, it is important that the parameters produced by a particular image processing technique for the characterization of ice accretions have clear physical interpretations. Parameters with

ambiguous meanings are much more difficult to implement in a fabrication process 


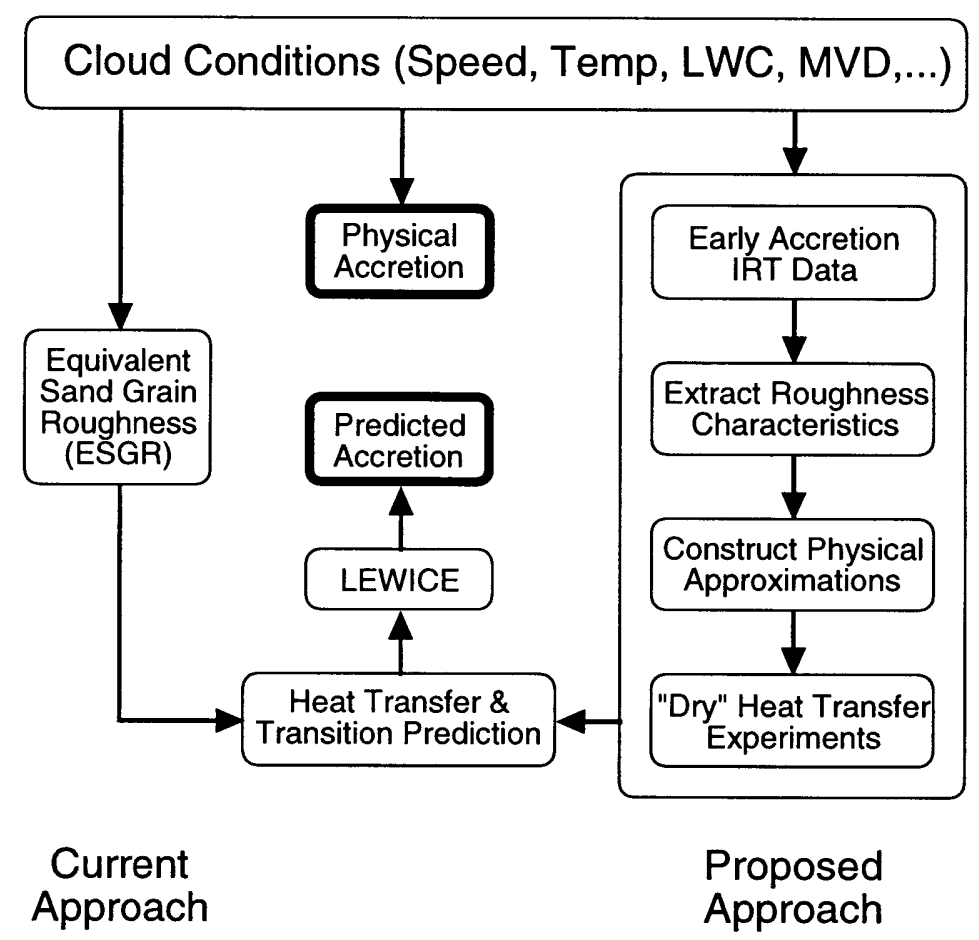

Figure 1-1: Schematic of current and proposed heat transfer model in LEWICE.

for physical roughness samples to be used in wind tunnel heat transfer testing. In the current context, a prospective image processing technique is considered feasible only if it is both efficient (low number of parameters) and readily implemented into the proposed approach of Figure 1-1.

Once the pertinent roughness characteristics have been extracted from experimental images, the final missing link in the proposed heat transfer model is obtained by incorporating this information into physical roughness samples. Construction of these roughness specimens represents the "Construct Physical Approximations" module of Figure 1-1.

Application of the techniques of Henry et al.[4], identified as the " 'Dry' Heat Transfer Experiments" module of Figure 1-1, completes a self-consistent scheme which connects close-up IRT video images to enhanced heat transfer observed on "similar" artificial roughness in dry wind tunnel tests. Similarity between experimentallyobserved surface roughness and artificial roughness is defined in terms of the selected image processing scheme.

\subsubsection{Outline}

With this methodology in mind, the thesis begins with an overview of available data followed by a review of two image processing techniques, the application of the selected image processing technique to close-up IRT icing images and the introduction of a method for creating images based upon analysis of experimental images. Next, a process for constructing physical roughness models suitable for heat transfer tests in 
a wind tunnel is demonstrated. The study concludes with the observation of enhanced heat transfer on physical samples in dry wind tunnel tests. 


\section{Chapter 2}

\section{Sample Images}

One of the principal shortcomings of ice accretion computer codes is a lack of traceability to real world ice accretions. In cases where the basic physics of ice formation are well understood and readily modeled, such as in rime ice, icing codes produce realistic final accretion shapes given a particular set of cloud conditions. However, under circumstances where it is difficult to proceed from the basic physics of ice formation to a reasonable computer model, as is the case with glaze ice, it is essential to refer to experimental data for guidance. Although IRT experiments have provided much basic information and intuition on the process of ice formation on aerodynamic surfaces, we feel that it is possible to take the usage of experimental data to a new level. Specifically, close-up video images and castings of glaze accretions provide the basis for obtaining quantitative information on early stage surface roughness and the accompanying enhancement of convective heat transfer. We begin development of this idea by giving an overview of the available experimental data.

\subsection{NASA Lewis IRT Video Data}

The image processing techniques[12] used in this study are applied to two sets of data. The first image set is the high-magnification video data taken from ice accretion experiments described by Hansman et al.[5] in which a 4" diameter cylinder (faired in the back) was exposed to the icing cloud over a wide range of wind speeds, temperatures and liquid water content. Simultaneous video images of both the overall and stagnation region (taken with a long-focal-length lens stationed in the IRT control room) were taken during that experiment, although only the close-up images are used in this study. The experimental set-up is shown in Figure 2-1.

Figures 2-2 and 2-3 show a typical time sequence of glaze and mixed accretions, respectively. The brightly lit areas can be interpreted as ice bumps which reflect the illuminating light while the dark areas are the valleys between roughness elements. This interpretation is rather subjective since other geometric features, such as glare from the model (which was aluminum) or reflection by surface water, might give a misleading impression of the surface texture. Nevertheless, the image is descriptive of the accretion physics although the interpretation must proceed with caution. Note the 


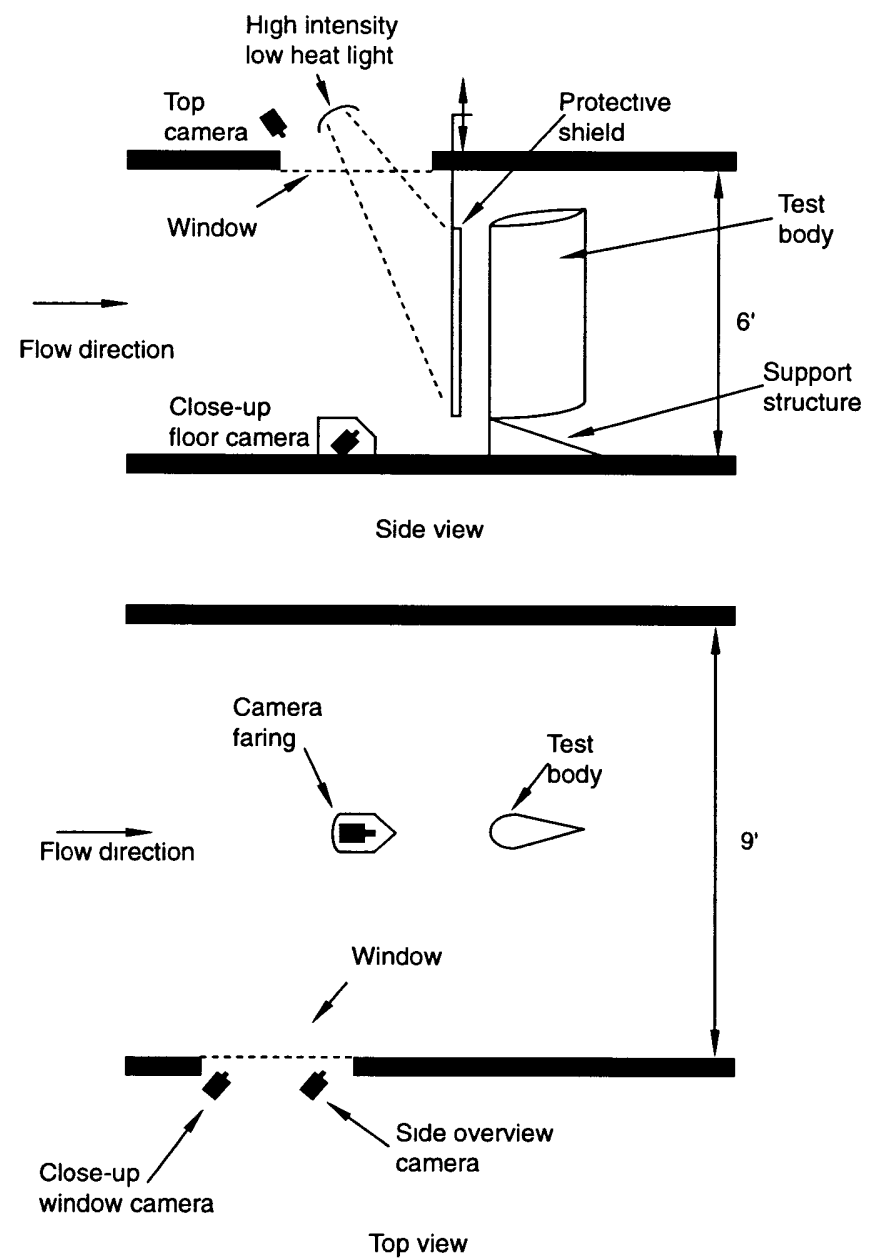

Figure 2-1: Schematic of IRT setup. Close-up images analyzed in the current study are acquired from the close-up window camera. Figure reproduced from Hansman et al. [5].

bead-like character of the mixed case in Figure 2-3. Early glaze and mixed accretions are dominated by the presence of these localized ice growths. Although the ice bumps are not as clearly defined in the glaze case as they are in the mixed case, it is also apparent that there does seem to be a characteristic range of bump sizes in the glaze image. Additional IRT video images are documented in the appendices.

\section{$2.2 \quad$ Icing Roughness Castings}

The second image set is derived from the models used in the wind tunnel heat transfer experiments of Masiulaniec et al.[8] These models consist of castings from flat plate ice shapes taken at the NASA Lewis IRT. In the IRT experiments that produced the castings, a flat plate was exposed to an icing cloud which produced ice roughness that varied primarily with streamwise location on the plate. Once an accretion was 
$1 \mathrm{sec}$.

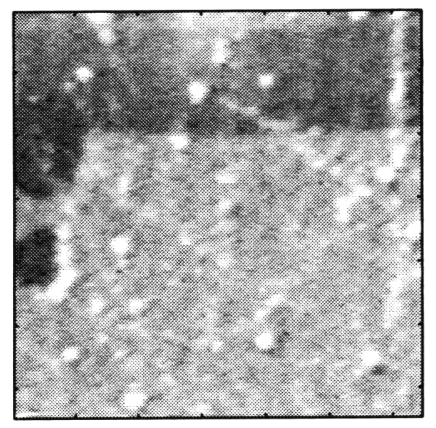

$10 \mathrm{sec}$.

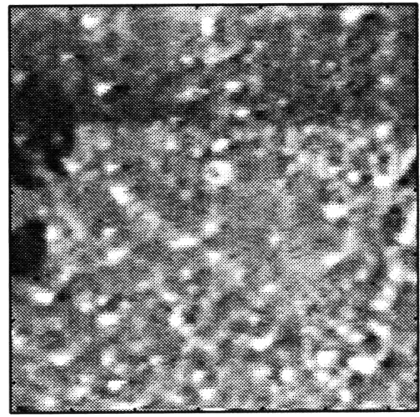

$20 \mathrm{sec}$.

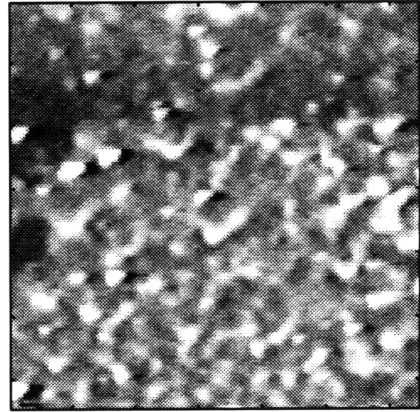

Figure 2-2: Time sequence of glaze accretion. Dark regions in upper portion of images are from markings on the wind tunnel model and were cropped out of data used for streamwise calculations. Field of view is approx. $1 \mathrm{~cm} \times 1 \mathrm{~cm}$. Cloud conditions: $T=25^{\circ} \mathrm{F}, U=150 \mathrm{mph}, L W C=1.0 \mathrm{~g} / \mathrm{m}^{3}, M V D=20 \mu \mathrm{m}$

$1 \mathrm{sec}$.

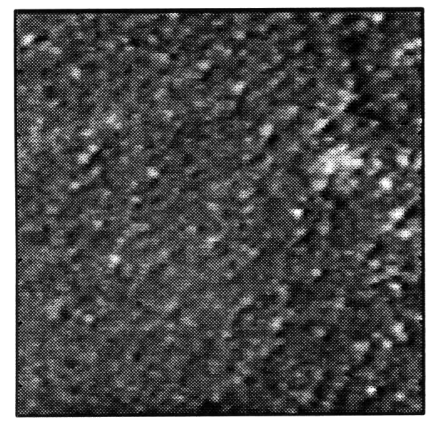

$10 \mathrm{sec}$.

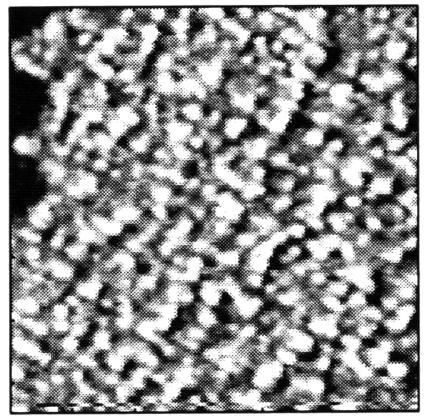

$20 \mathrm{sec}$.

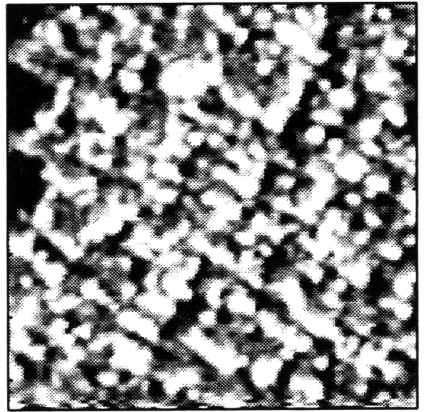

Figure 2-3: Time sequence of mixed accretion. Dark regions in upper portion of images are from markings on the wind tunnel model and were cropped out of data used for streamwise calculations. Field of view is approximately $1 \mathrm{~cm} \times 1 \mathrm{~cm}$. Cloud conditions: $T=25^{\circ} \mathrm{F}, U=100 \mathrm{mph}, L W C=0.7 \mathrm{~g} / \mathrm{m}^{3}, M V D=15 \mu \mathrm{m}$

established, the experiment was halted and an investment casting was made of the surface. These large castings were then partitioned into smaller sectors whose dimensions were sufficiently small so that the icing roughness was qualitatively similar within each particular sector.

Shown in Figure 2-4, the casting was taken at a more advanced stage in the accretion when many of the interstices between the initial ice beads had been filled with accumulations of additional ice causing some of the early bead-like structures to merge into larger roughness elements of complex connectivity. Consequently, one might expect dry heat transfer tests on such roughness samples to exhibit the "closepacked" constant heat transfer enhancement behavior observed by Henry et al. [4] in cases of high density roughness element arrays. Although the maturity of the ice accretions captured by the castings is contradictory to the goal of studying early stage 


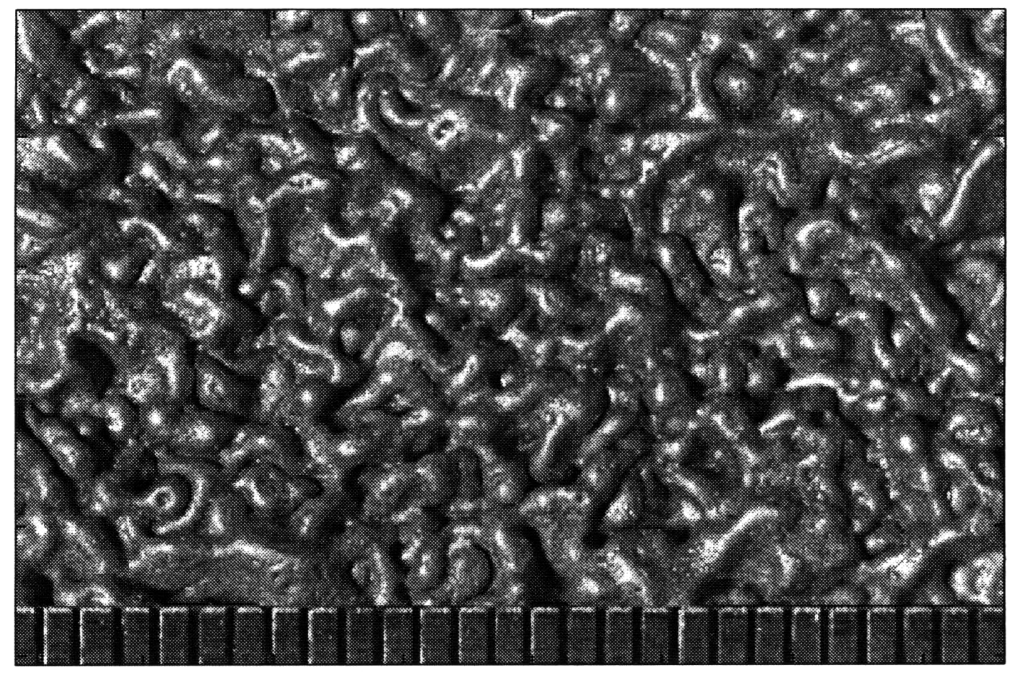

Figure 2-4: Surface roughness casting image from a model used in the heat transfer experiments of Masiulaniec et al.[8] Scale on the image is in $\mathrm{mm}$.

roughness, the castings provide an opportunity to conduct comparative heat transfer studies on actual ice shapes and the corresponding "similar" artificial roughness samples. The NASA IRT video images of glaze and mixed accretions are more representative of early stage roughness, but no roughness castings exist at early stages $(\sim 1 \mathrm{~min}$.$) since the experiments were also designed to investigate long term (\sim 10$ min.) ice shapes. 


\section{Chapter 3}

\section{Markov Random Field Texture Analysis}

Usage of IRT video images and castings to improve current icing codes is only as effective as the technique used to extract quantitative information from the experimental data. Because the field of texture analysis and pattern recognition is still under development, it is not obvious, a priori, if any of the current image processing techniques will be adequate. There do, however, exist promising techniques in the current literature such as the method examined below.

Because early glaze and mixed accretions are dominated by localized growths of ice (see Figures 2-2 and 2-3), an image processing technique which can quantitatively model discrete structures is attractive. Furthermore, the importance of roughness element size and spacing observed in the dry heat transfer experiments of Henry et al.[4] indicates that a "clustering" description of early icing roughness may be of considerable value. Markov Random Field (MRF) texture modeling[2] is just such a scheme. Using a stochastic simulation, MRF analysis provides a quantitative description of clustering present in a given ice accretion roughness texture field. In evaluating the feasibility of the MRF technique as an option for introducing experimental data into the improved heat transfer model of Figure 1-1, one must be careful to consider efficiency (low number of parameters) vis-á-vis ease of implementation; a roughness description that is efficient but not amenable to practical implementation is inefficacious.

\subsection{Informal MRF Overview}

The MRF model has its roots in the Monte Carlo simulations of random defects in crystal lattices that were published in the 1950's[9]. In particular, the MRF was used in the Ising model of statistical mechanics. Consequently, virtually all aspects of the texture generation process have rigorous thermodynamic analogies and for explanatory purposes, it is useful to describe the MRF model in terms of its original crystal lattice applications. In the discussion below, we follow the development of Picard[14]. 
One can interpret each pixel location in an image as a "site" in a crystal lattice and each pixel as an "atom" located at that site. The gray level of each pixel corresponds to the atom's species. Thus a $256 \times 256$ pixel, $8-b i t$ gray-scale image represents $65536=256^{2}$ atoms on a regular lattice in which each atom can be one of $256=2^{8}$ different species.

We further assume that the lattice is at some temperature which determines an average energy level on the lattice. If the temperature is high enough, all of the atoms will be in a liquid phase, free to move from one site to another, with their motion determined by random "vibrations" as well as attractive and repulsive forces between different species of atoms. As the temperature is lowered (according to some prescribed annealing schedule) atoms begin to precipitate out and assume fixed positions in the lattice. The probability that a particular type of atom will occupy a given lattice site is dependent on what types of atoms occupy the nearest neighbor sites. If a repulsive force exists between a given atom and its dissimilar neighbors, then occupation of the site by that particular species is unlikely (although possible). As the annealing temperature decreases, atoms precipitate out of liquid phase to form clusters which, while generated by random seeds, evolve in such a way so as to minimize the cluster boundaries for attractive atoms and to maximize the cluster boundaries for repulsive atoms. A rapid drop in annealing temperature corresponds to a quenching process which does not allow for optimal ordering of the crystal boundaries while a slow anneal allows for atoms to move to more optimal sites and results in a more ordered clustering.

In the MRF image processing scheme presented below, the "annealing process" that forms texture clusters takes the form of a Monte Carlo simulation that interchanges pixels of different gray-scale levels depending on whether or not the neighbors at the prospective site are similar in species. The "temperature level" of the image is the number of successful interchanges that occur on a given Monte Carlo iteration. The texture clusters that emerge from the annealing process are described by clustering parameters that represent the annealing rates of the lattice atoms in various directions within the lattice.

\subsection{Introduction to Stochastic Texture Modeling}

In general, most texture research can be understood using one of two viewpoints[2]. The first viewpoint makes the stochastic assumption that the image is a member of a probability sample space. The image usually takes the form of a discrete rectangular grid in which the values stored at each grid point are treated as random variables. The second viewpoint describes textures in terms of simple primitive objects. Complex textures are built by the placement of the primitive objects at possibly different scales. Although textures described by the placement rule viewpoint may have some random aspects, the primary building blocks of the texture are uniform in shape. In contrast, textures described by the stochastic rule are composed of elements which are varied in shape and lack intuitive description.

One texture modeling scheme which applies the stochastic viewpoint is MRF 
texture analysis. With this technique, the image is represented as an array of pixels, each of which is treated as a random variable that may assume values corresponding to the gray-scale levels possible. Intuitively, it is clear that the value of a given pixel will be influenced by the values of its neighbors. For example, if a pixel is located in the center of a lightly colored structure, then there is a high probability that the pixel in question will have a light shade of gray unless, of course, the image contains an element of random noise. Thus, the probability of a given pixel assuming a given level of gray is conditional on the gray levels "around" it. The MRF technique simulates the texture as a conditional probability model for a finite system of spatially interacting random variables.

Before a computational scheme can be implemented, several key aspects of the model that affect the representation of icing roughness "clustering" must be developed. First of all, one must formulate a precise definition of "neighbor." To associate a pair of pixels located within the same ice bead, such a concept may include the notion of close spatial proximity in the image to the site in question. However, this need not be the case, especially near the boundaries.

Secondly, to specify the mathematical relation between the pixels in each ice bead, it is necessary to select what type of random variables will represent the pixels. For example, one must decide if the pixel gray levels will obey distributions corresponding to exponential, binomial, Poisson, gamma, or some other type of random variable.

Thirdly, one must cope with the fact that for a random variable spatial interaction law determined by the definition of neighbor and the type of random variables used, it is difficult to generate an image that is representative of the interaction law using brute force techniques that exhaust the sample space. When an MRF image is generated, one must determine how likely that image is under the constraints of the spatial interaction law applied to each pixel and its neighbors. If the pixel gray levels were chosen randomly, then the likelihood of such an image occurring in a sample space governed by a specific conditional distribution is extremely low. Since the goal of the analysis is to generate different MRF images by altering the governing interaction law, it is desirable to produce images which are "representative" of the selected interaction law. Consequently, image generation hinges on one's ability to find high probability images in a vast image sample space using simulation techniques rather than exhaustive searches.

\subsection{MRF Framework for Image Generation}

The following definitions are from the development of Cross and Jain [2]. Let $X(i, j)$ be the gray level at site $(i, j)$ on the $N \times N$ lattice $L$. Re-index $X(i, j)$ to $X(i)$ where $i=1,2, \ldots M ; M=N^{2}$.

Definition 1: Let $L$ be a lattice. A coloring of $L$ with $G$ levels, denoted $\mathbf{X}$, is a function from the points of $L$ to the set $\{0,1, \ldots, G-1\}$.

Definition 2: The sample space of all possible colorings of $L$ with $G$ levels is denoted by $\mathcal{L}$. 
Definition 3: The point $j$ is said to be a neighbor of the point $i$ if

$$
p(X(i) \mid X(1), X(2), \ldots, X(i-1), X(i+1), \ldots, X(M))
$$

depends on $X(j)$.

Definition 4: A Markov random field is a joint probability density on the set $\mathcal{L}$ subject to the following conditions:

1. Positivity: $p(\mathbf{X})>0 \forall \mathbf{X} \in \mathcal{L}$

2. Markovianity:

$$
p(X(i) \mid \text { all points in } L \text { except } i)=p(X(i) \mid \text { neighbors of } i)
$$

3. Homogeneity: $\quad p(X(i) \mid$ neighbors of $i)$ depends only on the configuration of neighbors and is translation invariant for translations involving the same neighborhood configuration.

\subsubsection{Random Variable Selection}

For the model used in this study, the discrete conditional probability distribution for the gray level of each pixel is that of the binomial random variable. That is, for each pixel in the image, the gray level is governed by:

$$
p(X(i)=k \mid \text { neighbors })=\left(\begin{array}{c}
G-1 \\
k
\end{array}\right) \theta^{k}(1-\theta)^{G-1-k}
$$

where $\theta$ is the parameter of the random variable. In terms of the classical coinflipping analogy, each pixel is a $B(G-1, \theta)$ binomial random variable where $G-1$ is the number of "flips" and $\theta$ is the probability that any one flip of the coin will produce a "head." Therefore, $p(X(i)=k \mid$ neighbors $)$ is the probability that one will observe $k$ heads during $G-1$ flips of a $\theta$-coin. Note that the coin need not be "fair," so that in general, $\theta \neq 0.5$.

In practice, it is more efficient from a computational standpoint to compute the logarithm of the binomial probability:

$$
\log (p(X(i)=k \mid \text { neighbors }))=\sum_{n=1}^{k} \log (G-1-k+n)-\sum_{n=1}^{k} \log (n)+k \log (\theta)+(G-1-k) \log (1-\theta)
$$

\subsubsection{Besag Codings and Classification of Markov Random Fields}

Although the framework for image generation outlines the role of random variables in the MRF technique, it was still necessary to specify what particular type of random variables would be used in the numerical scheme (see Equation 3.1). Similarly, the 


\begin{tabular}{|c|c|c|c|c|}
\hline & 01 & $m$ & $q 1$ & \\
\hline 02 & $v$ & $u$ & $z$ & $q 2$ \\
\hline$l$ & $t$ & $x$ & $t^{\prime}$ & $I^{\prime}$ \\
\hline$q 1^{\prime}$ & $z^{\prime}$ & $u^{\prime}$ & $v^{\prime}$ & $01^{\prime}$ \\
\hline & q2' & $m^{\prime}$ & $02 '$ & \\
\hline
\end{tabular}

Figure 3-1: Besag codings: neighbors of the point $x$.

rather general definition of the neighbors of a pixel requires an explicit relation in order to be implemented computationally. Classes of such relations were developed by Besag [1] and implemented by Cross and Jain [2].

Given a pixel location (or, equivalently, a random variable), $X$, enumerate the surrounding pixels as shown in Figure 3-1. The neighbors of $X$ influence the corresponding gray level by determining the parameter, $\theta$, of the binomial random variable in Equation 3.1 represented by $X$. The parameter $\theta$ is calculated as follows:

$$
\theta=\frac{e^{T}}{1+e^{T}}
$$

where $T$ takes the following form for a first order neighborhood (all neighbors defined in Figure 3-1):

$$
T=a+b(1,1)\left(t+t^{\prime}\right)+b(1,2)\left(u+u^{\prime}\right) .
$$

A second order neighborhood has a $T$ of the form:

$$
T=a+b(1,1)\left(t+t^{\prime}\right)+b(1,2)\left(u+u^{\prime}\right)+b(2,1)\left(v+v^{\prime}\right)+b(2,2)\left(z+z^{\prime}\right) .
$$

A third order neighborhood utilizes the second order value of $T$ plus the additional terms:

$$
b(3,1)\left(m+m^{\prime}\right)+b(3,2)\left(l+l^{\prime}\right) .
$$

A fourth order neighborhood uses the value of $T$ from the third order neighborhood plus the additional terms:

$$
b(4,1)\left(o 1+o 1^{\prime}+o 2+o 2^{\prime}\right)+b(4,2)\left(q 1+q 1^{\prime}+q 2+q 2^{\prime}\right) .
$$

Neighborhoods of the above forms are known as Besag codings. The $b(i, j)$ are called clustering parameters and form the basis for classifying the various Besag codings. Positive values of $b(1,1)$ cause texture clustering in the horizontal direction while 
positive values of $b(1,2)$ produce clustering in the vertical direction. Negative values of the $b(1, i)$ produce repulsion of texture structures in the corresponding directions. Similarly, the $b(2,1)$ and $b(2,2)$ control clustering in the diagonal directions, producing diagonal structures in the case of attraction and checker-board effects in the case of repulsion. More complicated forms of anisotropy can be produced by more complicated neighborhood configurations and nonlinear expressions for $T$.

The order of a Markov random field process on a lattice is the largest value of $i$ such that $b(i, 1)$ or $b(i, 2)$ is nonzero. A Markov random field is said to be isotropic at order $i$ if $b(i, 1)=b(i, 2)$. Otherwise, it is said to be anisotropic at order $i$. The symbol $b(i, \cdot)$ signifies isotropy at order $i$ and takes the common value of $b(i, 1)=b(i, 2)$.

\subsubsection{MRF Simulation: The Method of Rejection/Selection}

Markov random fields represent a complicated joint probability structure established upon an image sample space, $\mathcal{L}$. When considering MRF textures on a given lattice of random variables, $\mathbf{X} \in \mathcal{L}$, it is useful to know how "representative" $\mathbf{X}$ is of a random sample of images from $\mathcal{L}$. By the positivity condition of Markov random fields, it is clear that all lattice colorings obtainable by the range of permissible random variable gray levels must be possible, however unlikely. Because the clustering parameters determine the character of textures in a given sample space, some images in the sample space must be more probable than the others in order to distinguish "characteristic" images for the given set of $b(i, j)$. In other words, representative images will be those for which $p(\mathbf{X})$ is maximized for a fixed value of $\theta$ (Equation 3.3).

In fact, estimation of the clustering parameters of an experimentally obtained image is done in this fashion, known as a maximum likelihood estimate. For a given image and an assumed joint probability structure (in this case binomial random variables), the determination of the binomial parameter $\theta$ that maximizes the probability of the given image being randomly sampled from $\mathcal{L}$ requires solving an optimization problem over the clustering parameters due to the dependence of $\theta$ on $T$ via $a$ and the $b(\cdot, \cdot)$. This is a very difficult optimization problem and requires sophisticated techniques for neighborhood definitions of modest complexity. For example, a $4^{\text {th }}$ order model requires optimizing over nine parameters. The method of optimization employed in this study in that of simulated annealing as applied by Press et. al [15]. In passing, it should be mentioned that from a computational standpoint, it is much more convenient to maximize a log-likelihood function similar to that given in Equation 3.2.

Ideally, one could derive an analytical scheme for directly determining the image $\mathbf{X}$ that is most likely to be obtained in a random sample of $\mathcal{L}$. When one notices that the condition of Markovianity required for Markov random fields can, in the case of a simple neighborhood definition, place significant restrictions on the size of an image sample space, such an analytical scheme might seem feasible. This may be possible for special cases of Markov random fields on simple lattices, but in the general setting of a large lattice, arbitrary random variables at the lattice sites, complicated neighborhood schemes, and a large range of gray levels available, the possibility of such an analytical scheme seems remote. 
Another approach to finding the characteristic $\mathrm{X}$ of the $b(i, j)$ is to select a random image from $\mathcal{L}$, compute $p(x \mid$ neighbors $)$ for every lattice site and then compute the conjunctive probability of all the individual lattice events. Next, an alteration must be made to the pixel gray levels in the image so that $p(x \mid$ neighbors $)$ is increased. This process must be repeated until the maximal conjunctive probability is obtained in an expedient fashion. Although this scheme seems more promising than the first approach, a convergent technique for correcting the images on successive iterations must be implemented. To see how such a technique may be developed, we follow the exposition of Ross [16].

Suppose that a method for simulating a random variable $Y$ with density function $g(x)$ is available. Let $X$ be a random variable with density function $f(x)$ that we desire to simulate. It is possible to use the capability to simulate $Y$ in the simulation of $X$. The process begins by simulating $Y$ with density $g$. A random number $U$ is then simulated. Let $c$ be a constant such that

$$
\frac{f(y)}{g(y)} \leq c \forall y .
$$

$Y$ is accepted as a valid value of $X$ with a probability proportional to $f(Y) / g(Y)$. The process is outlined as follows:

1. Step 1: Simulate $Y$ having density $g$ and simulate a random number $U$.

2. Step 2: If $U \leq f(Y) / c g(Y)$, set $X=Y$. Otherwise, return to Step 1 .

This scheme is known as the method of selection/rejection and is explained in detail in the appendices.

\subsubsection{Practical MRF Simulation}

The actual implementation of the method of rejection/selection requires only the calculation of the ratio $f(Y) /(c g(Y))$. It is not necessary to be able to compute $f(Y)$ and $g(Y)$ separately. Moreover, since the MRF simulations will be on a finite lattice, we replace the continuous random variables in the simulation technique with their discrete analogues. If $\mathbf{X}$ and $\mathbf{Y}$ are any two lattice colorings in $\mathcal{L}$, then the ratio we seek for the simulation if $p(\mathbf{X}) / p(\mathbf{Y})$. Note that discrete probability mass functions have replaced continuous density functions.

Besag [1] derived a simple formula for calculating $p(\mathbf{X}) / p(\mathbf{Y})$ :

$$
\frac{p(\mathbf{X})}{p(\mathbf{Y})}=\prod_{i=1}^{M} \frac{p\left(x_{i} \mid x_{1}, \ldots, x_{i-1}, y_{i+1}, \ldots, y_{M}\right)}{p\left(y_{i} \mid x_{1}, \ldots, x_{i-1}, y_{i+1}, \ldots, y_{M}\right)}
$$

The full derivation is given in the appendices. In order to significantly decrease the required computations, Lindsay[7] showed that Besag's formula can be reduced to:

$$
\frac{p(\mathbf{X})}{p(\mathbf{Y})}=\frac{e^{y_{\circ} T_{x}} e^{x_{\circ} T_{y}}}{e^{x_{\circ} T_{x}} e^{y_{\circ} T_{y}}}
$$


where $x_{\circ}$ and $y_{\circ}$ are the gray-scale levels at the two lattice locations in question, and $T_{x}$ and $T_{y}$ are the neighborhood configuration parameters for $x$ and $y$ from Equation 3.3. The MRF code developed for this study uses Lindsay's equation. A comparative study between this code and the code of Cross and Jain[2] is given in the appendices.

\subsubsection{Estimation of Clustering Parameters}

The inverse process of image generation, that of taking an existing image and backing out the clustering parameters, is a straightforward but computationally intensive maximum likelihood estimate based upon the binomial model selected for the current study. Specifically, the log-likelihood function of Equation 3.2 must be optimized for the binomial parameter, $\theta$. Since $\theta$ depends upon the complexity of the neighborhood scheme, complicated neighborhoods lead to difficult estimations of the binomial parameter. For example, the rather modest fourth-order model used in this work requires nine parameters to statistically describe an image. Obtaining these parameters thus requires the maximization of a nine-dimensional function, resulting in a very large search domain.

Since it's unclear what properties the log-likelihood function will have (for example, differentiability) for an arbitrary neighborhood definition, derivative-based optimization schemes such as conjugate-gradient may not be applicable. Instead, the more general, Monte Carlo-based simulated annealing optimization scheme[15] was selected. The accuracy of parameter estimation for low-order models to within the 5 percent tolerance quoted by Garand[3] has been confirmed. Identical textures for the same parameter values investigated by Cross and Jain[2] have also been replicated. However, the parameter estimation process is somewhat problematic, an issue discussed in more detail in the "Discussion" section of this chapter.

\subsection{MRF Analysis of Accretion Images}

To demonstrate the MRF technique, the clustering parameters were estimated from the IRT glaze ice image shown in Figure 3-2 (a). For this case, the cloud conditions were $U=100 \mathrm{mph}, T=25^{\circ} \mathrm{F}, L W C=1 \mathrm{~g} / \mathrm{m}^{3}, M V D=15 \mu \mathrm{m}$. The initial condition of white noise (Figure 3-2 (b)) was used for image generation. The graininess of the image (especially compared with the images in the previous sections) is due to the fact that the resolution of the images was reduced to $128 \times 128$ pixels and that only a 4 -bit gray scale is used (rather than an 8-bit gray scale as in the previous images). Both of these simplifications were taken to reduce the computational requirements.

As shown in Figure 3-2 (c), the MRF model was able to reproduce a remarkably similar image from only nine constants taken from the original. This give a data compression factor of over 7000! The most noticeable differences between the simulation and the original image are the two large-scale, dark, vein-like structures that protrude into the original image from the right hand side. This highlights one of the principle shortcomings of the MRF texture model - that it is only able to characterize micro- 
scale textures that are translationally invariant in space. Since ice accretion images evolve in the stream-wise direction away from the stagnation region, the basic MRF model is unable to accurately capture the large scale features or spatial evolution present in the physical accretion images.

This difficulty has been solved by employing a "structural-stochastic" model [3] in which a gross spectral truncation of the original image is used as the input initial condition for the MRF model. Although the initial condition of the MRF model should not affect its final state, in practice, the large-scale features do persist, even after many Monte Carlo cycles. When this structural model is incorporated into the basic MRF algorithm (using $0.3 \%$ of the total Fourier modes in the initial image as the structural backbone), the synthetic accretion image obtained (Figure 3-2 (d)) is essentially identical in character to the experimentally observed ice image.

\subsection{Discussion}

The MRF model is attractive because one can estimate model parameters from experimental data and, in principle, generate a realistic looking image that closely resembles a physical accretion with a relatively small number of parameters. Thus, MRF texture analysis satisfies the previously stated efficiency requirement.

In practice, however, there are problems with both the estimation and generation phases of the calculation. For image synthesis, it is unclear how many Monte Carlo cycles are needed to create an image in statistical equilibrium. Current indications are that the images previously thought to be stable can still change substantially if more iterations are allowed. In the MRF literature, this is known as the "temperature problem" and has been addressed theoretically by Picard [14]. However, the issue is by no means settled and continues to present difficulties in image generation.

Estimation of parameters also presents some serious difficulties. Simultaneous optimization of nine parameters is difficult and computationally expensive. For low order models with only a few gray levels such as those considered by Cross and Jain [2], the parameter estimations seem reliable. However, for more realistic images which require higher order models and several gray levels, the estimation process is difficult and requires considerable user intervention.

The lack of a straight-forward physical interpretation of the clustering parameters leads one to suspect that implementing the MRF roughness characterization in the improved heat transfer model of Figure 1-1 will be difficult at best. Consequently, despite the fact that MRF texture analysis is capable of achieving a remarkably efficient representation of extremely complicated icing images, the dim prospects of ever being able to reliably estimate the parameters from a wide range of images and create physical roughness specimens based upon the MRF parameters renders the technique infeasible. 


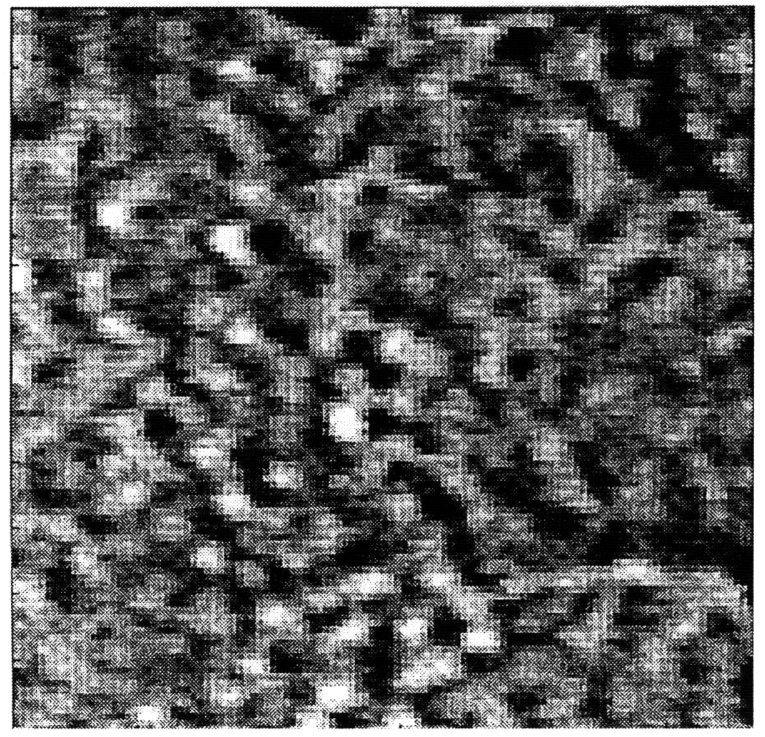

(a) Actual glaze accretion image used for MRF comparison.

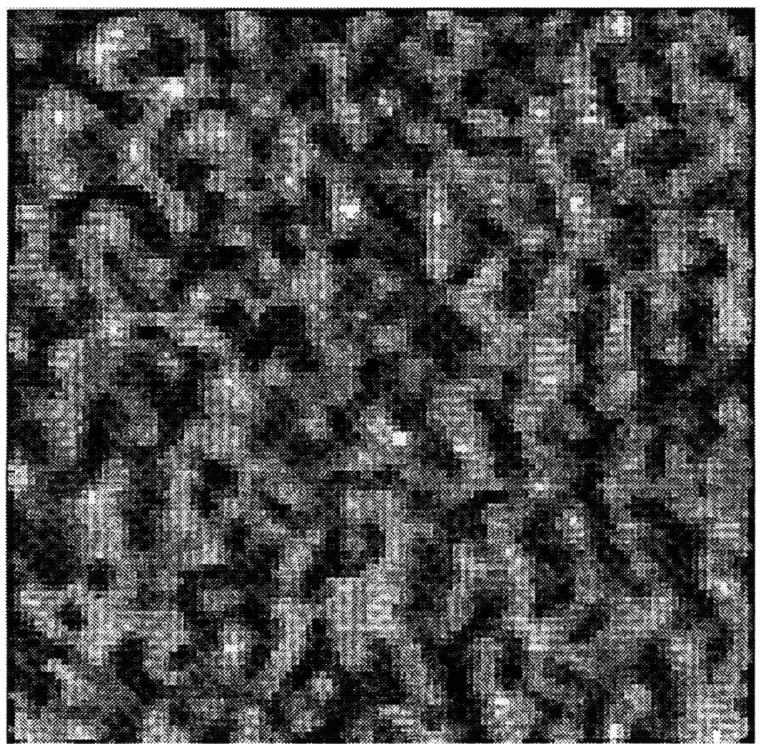

(c) Synthetic image of glaze accretion using basic MRF model.

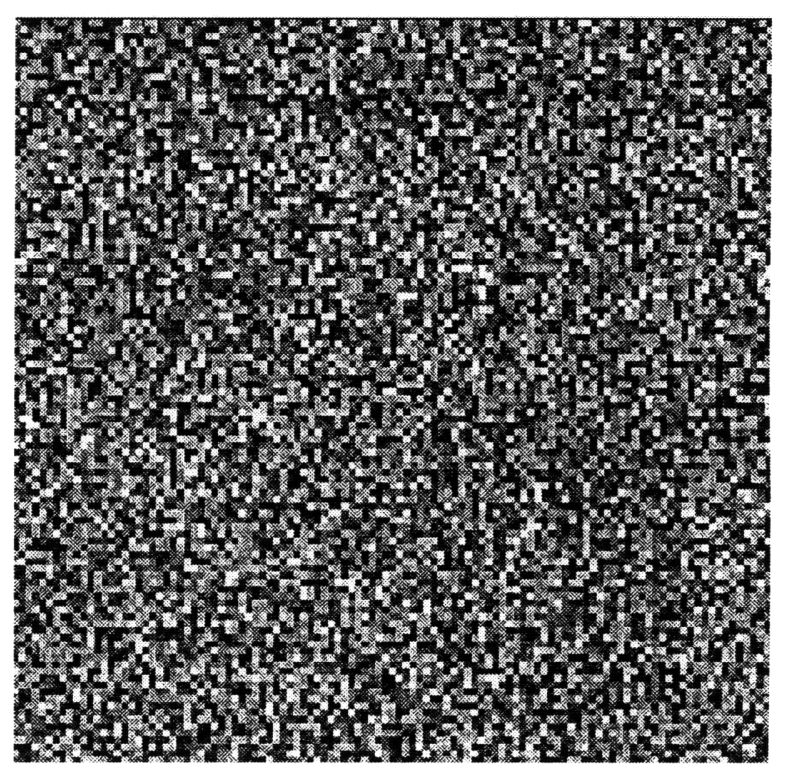

(b) Random pixel distribution used as initial condition for MRF calculation.

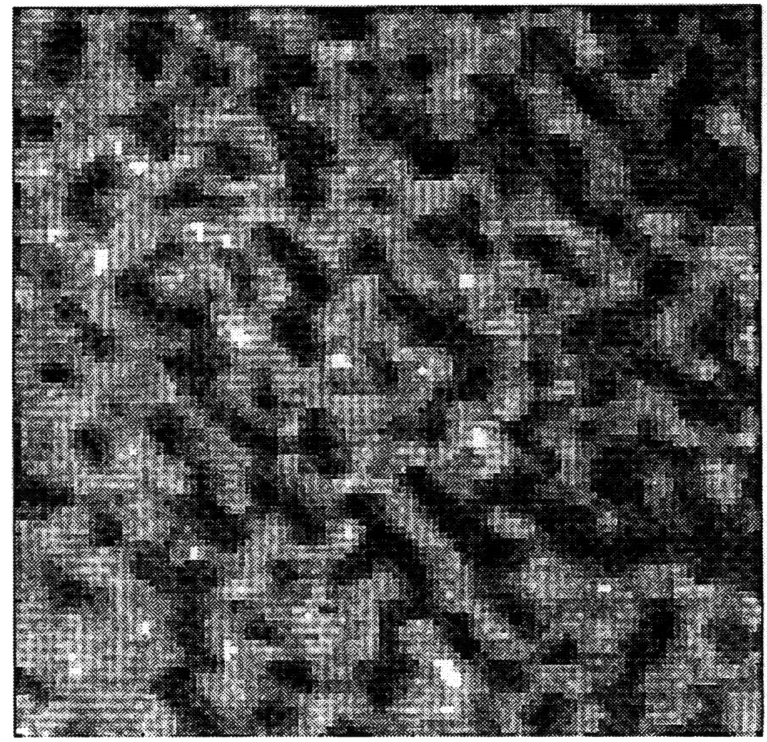

(d) Synthetic image using "structural-stochastic" MRF model.

Figure 3-2: MRF Simulation of Glaze Ice Accretion 


\section{Chapter 4}

\section{Spectral Techniques for Texture Analysis}

Although the MRF technique was very efficient in representing complex glaze accretions, it failed to meet the second basic requirement for image processing techniques in this study: facilitation of a fabrication technique for physical roughness samples appropriate for dry wind tunnel heat transfer tests. This requirement is a key step in completing the methodology shown in Figure 1-1. With this in mind, we now present an original primitive object placement rule image processing scheme specially developed for analysis of ice accretion images.

\subsection{Spectral Description of Roughness}

Figure 4-1 (a) shows a typical image of an early glaze accretion from the IRT closeup video data. The resolution of the images is $256 \times 256$ pixels with a scale of 30 pixels $/ \mathrm{mm}$. The cloud conditions are $U_{\infty}=150 \mathrm{mph}, T=25^{\circ} \mathrm{F}, L W C=1 \mathrm{~g} / \mathrm{m}^{3}$ and $M V D=20 \mu \mathrm{m}$.

One method for characterizing the roughness size is to use a spectral technique in an attempt to identify a characteristic length scale by locating a maximum peak in the spectral domain. The efficiency of the spectral representation of an ice image is illustrated by taking the image shown in Figure 4-1 (a), Fourier- transforming the image (in both the $x$ - and $z$-directions), "filtering" the image by retaining only $2 \%$ of the Fourier modes (which contain the majority of the total energy), and finally transforming the filtered spectrum back to the spatial domain. The filtered image is shown in Figure 4-1 (b) and clearly retains the essential features of the original image although the sharper details are washed out. This indicates that the essential character of the ice roughness can be captured by a relatively small number of Fourier modes and gives rise to some hope that a processing scheme based upon a spectral analysis might be successful in characterizing a complex image such as the given ice accretion with only a small number of parameters.

The work of Henry et al.[4] demonstrates the impact on boundary layer heat transfer of not only roughness element size but also the spacing between adjacent roughness 


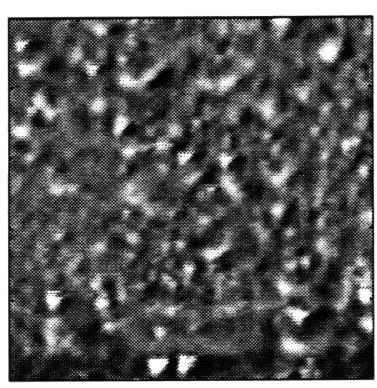

(a)

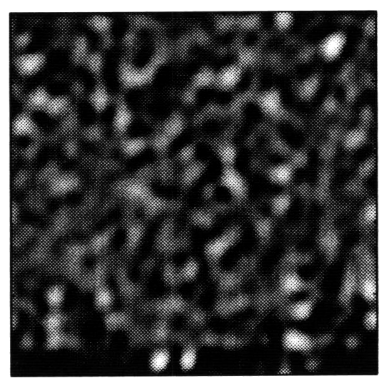

(b)

Figure 4-1: (a) Original image. (b) Filtered image with 2\% of modes retained.

elements. From the standpoint of developing the improved heat transfer model of Figure 1-1, it is reasonable to infer that the quantitative description of surface roughness necessary to incorporate experimental icing data into the scheme should be able to adequately capture aspects of roughness related to size and spacing. Furthermore, since heat transfer at the substrate provides the basis for additional ice growth, such added realism in the roughness model would likely result in a concomitant improvement in predicted ice accretions.

In light of this, we approach the characterization from a physical perspective. The physical approach is to compare the experimentally observed early ice accretion to an idealized "test function" which models the ice as a series of discrete "bumps" with a typical size, $D$, separated by a typical spacing, or period $P$. To avoid having to repeatedly translate the test function for fixed values of $D$ and $P$ in order to check the fit to the experimental data, we perform the quantitative analysis in the spectral domain where phase information is easily separated from the test function and discarded. In this way, for a given test function, we can use spectral methods to more efficiently find the values for $D$ and $P$ that optimally match the experimental image. Consequently, instead of trying to characterize the ice accretion spectrum in terms of a few Fourier modes, it is characterized by an ensemble of modes which combine to produce physically meaningful length scales. In terms of the primitive object viewpoint of texture analysis described earlier, the test function, quantified by $D$, plays the role of the primitive object while the spacing, quantified by $P$, represents a placement rule for the object.

\subsection{Spectral Estimation Technique}

\subsubsection{Basis}

To implement a placement rule approach for texture analysis, we consider the following scheme known as the spectral estimation technique (SET). The test function can, in principle, be arbitrary but for the present analysis, the function chosen consists of 


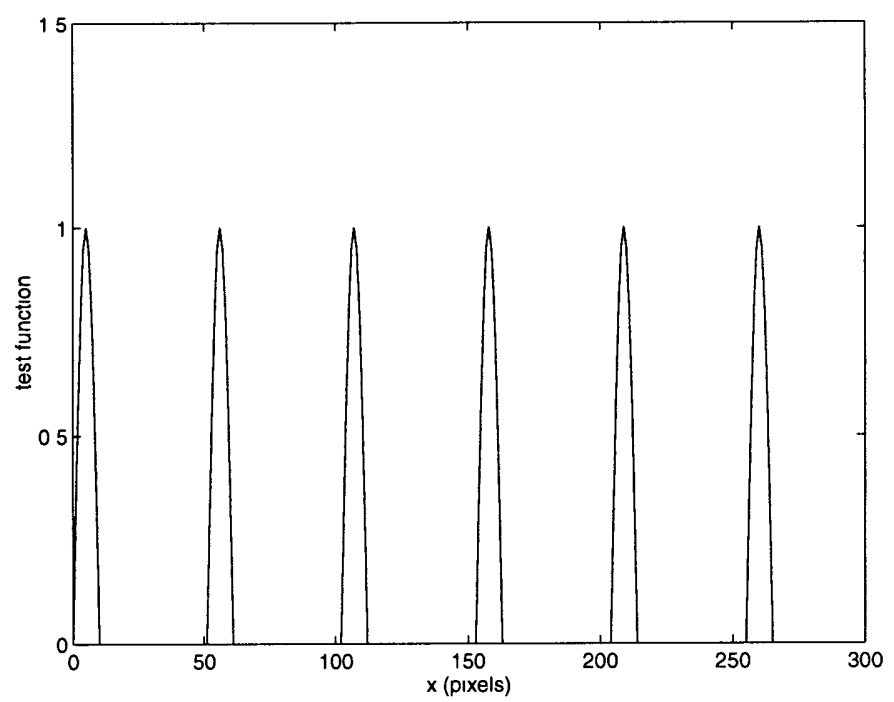

Figure 4-2: Sample test function for $D=12, P=51$.

a periodic extension of sinusoidal "beads":

$$
F_{\text {test }}(x)= \begin{cases}\sin \left(\frac{\pi x}{D}\right) & x=0 \ldots D \\ 0 & x=D \ldots P\end{cases}
$$

where $D$ is the width of the bead and $P$ is the period of the pattern.

An example of the test function is shown in Figure 4-2, for the choice of $D=$ 12 pixels, $P=51$ pixels. Although we have fit only the bead width and spacing, other shape parameters such as "roundness," symmetry, etc. could also be included without any difficulty although the computational resources required for the optimization will necessarily increase. As described, the test function is used to model only horizontal (streamwise) scan lines in the images. It is expected that the bead geometry will be different in the streamwise and spanwise directions and, for this reason, one should ideally employ a two-dimensional test function and optimize for the roughness characteristics in both the spanwise and streamwise directions on the model surface. This extension, which would utilize the full two-dimensional power spectrum, is not difficult but was not used in the current study in order to simplify the initial investigation and to reduce the parameter space for the optimization procedure.

\subsubsection{Algorithm}

Having defined the general test function, the procedure for optimizing $P$ and $D$ is as follows:

1. The power spectrum $(Q)$ of a single horizontal scan line of experimental data was computed using Fast Fourier Transform (FFT) techniques $\left(Q(\lambda)_{\text {expt }}\right)$. 


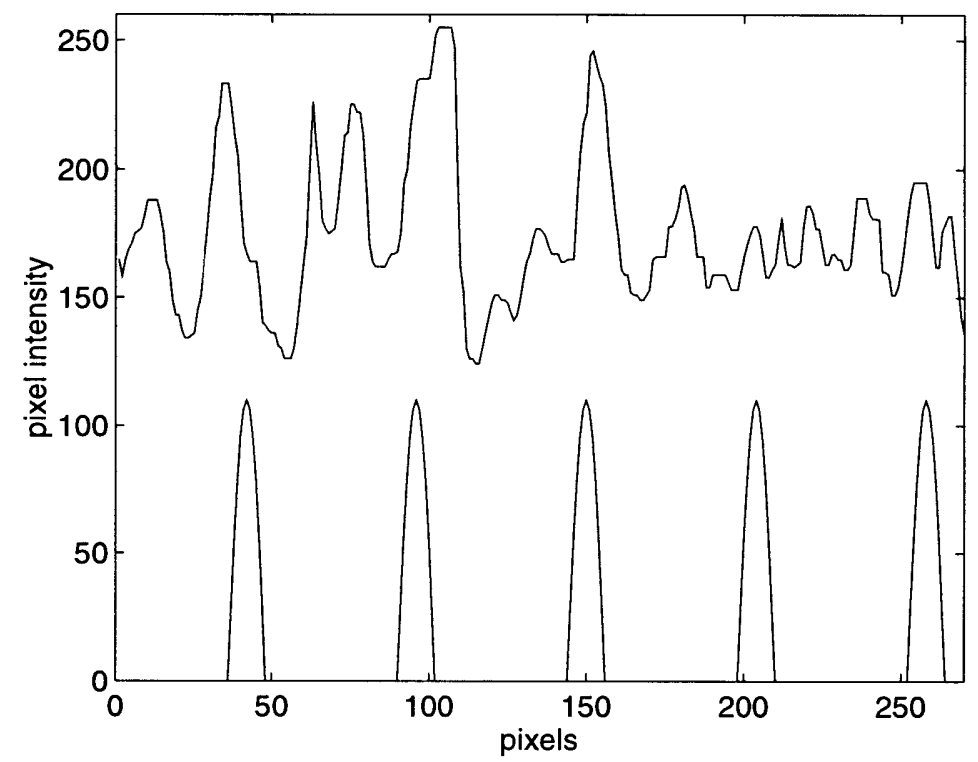

Figure 4-3: Comparison of experimental data and the corresponding optimal test function.

2. A $D-P$ combination was selected, the corresponding test function and its power spectrum was computed $\left(Q(\lambda)_{t e s t}\right)$.

3. The spectrum of the test function was normalized so that maxima of the two spectra were equal. This removed any bias due to uneven lighting or amplitude effects. The IRT video data was not calibrated for amplitude and for this reason, it was decided to normalize the image intensity so that differences in image intensity did not mislead the analysis. If an experimental image with a calibrated amplitude were available, this step would not be necessary but then the parameter search space would be expanded to include the roughness amplitude.

4. The "error" in the estimation was computed from the sum of the difference between the experimental and test spectra at each wavelength:

$$
\text { error }=\sum_{\lambda=\lambda_{\operatorname{m} \imath n}}^{\lambda_{\max }}\left|Q(\lambda)_{\text {expt }}-Q(\lambda)_{t e s t}\right|
$$

where $\lambda_{\min }$ and $\lambda_{\max }$ represent the minimum and maximum wavelength to be considered (typically $0.03-5.0 \mathrm{~mm}$ ).

5. The error was computed, as described, over all reasonable combinations of $D$ and $P$. The optimal combination was defined as those values of $D$ and $P$ 
resulting in the minimum value of the error.

Once the optimal $D, P$ combination for each image scan line has been determined, the results are aggregated into a single joint histogram over the $D-P$ domain.

\subsubsection{SET Refinements}

In order to improve the low-wavelength resolution of the spectrum, a multiple-radix FFT routine was used in the power spectrum computation, thereby allowing the number of pixels in each scan line to be comprised of any combination of powers of 2,3 , and 5 (rather than a power of 2 as is the case in conventional FFT routines).

Due to the simplicity of the test function, analytical expressions for the test function power spectrum may be used to expedite the calculations. The Fourier Series for the test function is:

$$
F_{\text {test }}(x)=\frac{a_{\circ}}{2}+\sum_{n=1}^{\infty}\left(a_{n} \cos \left(\frac{2 n \pi x}{P}\right)+b_{n} \sin \left(\frac{2 n \pi x}{P}\right)\right)
$$

where:

$$
\begin{gathered}
a_{\circ}=\frac{2}{P} \int_{0}^{P} F_{\text {test }}(x) d x \\
a_{n}=\frac{2}{P} \int_{0}^{P} F_{\text {test }}(x) \cos \left(\frac{2 n \pi x}{P}\right) d x \\
b_{n}=\frac{2}{P} \int_{0}^{P} F_{\text {test }}(x) \sin \left(\frac{2 n \pi x}{P}\right) d x
\end{gathered}
$$

For the given test function, $F_{t e s t}(x)$,

$$
\begin{gathered}
a_{\circ}=\frac{4 d}{P \pi} \\
a_{n}=\frac{2}{P}\left(\frac{1}{2 \omega_{1}} \cos \left(\omega_{1} d\right)-\frac{1}{2 \omega_{2}} \cos \omega_{2} d-\frac{1}{2 \omega_{1}}+\frac{1}{2 \omega_{2}}\right) \\
b_{n}=\frac{2}{P}\left(\frac{1}{2 \omega_{1}} \sin \left(\omega_{1} d\right)-\frac{1}{2 \omega_{2}} \sin \omega_{2} d\right)
\end{gathered}
$$

where:

$$
\begin{aligned}
& \omega_{1}=\frac{\left[(2 n-1)-\frac{P-d}{d}\right] \pi}{P} \\
& \omega_{2}=\frac{\left[(2 n+1)+\frac{P-d}{d}\right] \pi}{P}
\end{aligned}
$$

For $n=0,1,2, \ldots$, the Fourier amplitudes are then computed as:

$$
c_{n}=\sqrt{a_{n}^{2}+b_{n}^{2}}
$$

Of course, the number of modes kept in the above expressions is determined by the Nyquist frequency of the experimental scan lines that are to be fit by $F_{\text {test }}(x)$. 


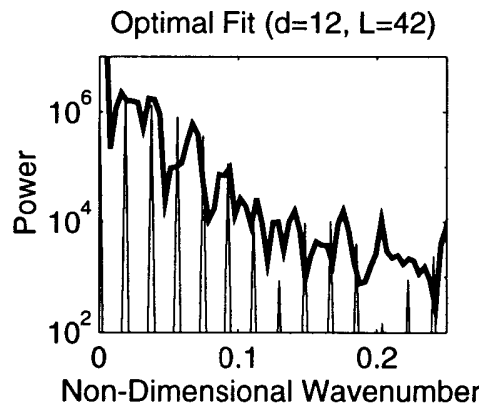

Non-optimal Fit $(d=6, L=42)$
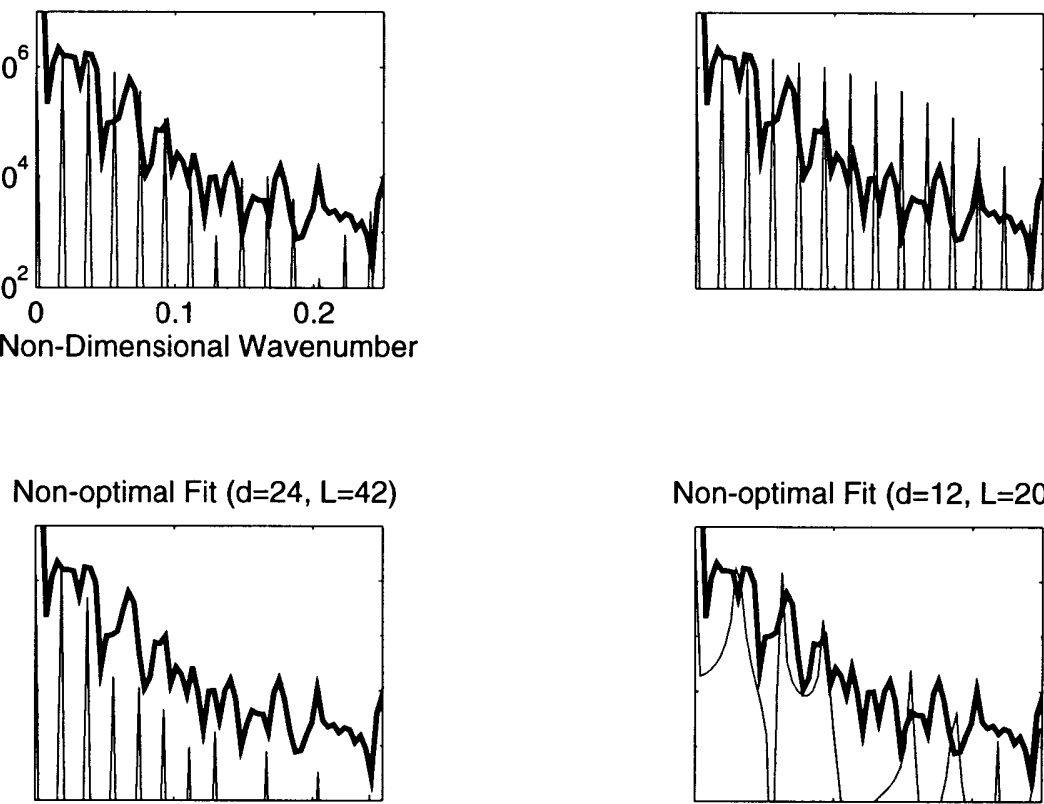

Non-optimal Fit $(d=12, L=20)$

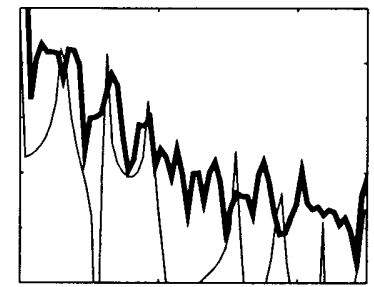

Figure 4-4: Effects of $P$ and $D$ on test function optimization. (upper left) optimal; (upper right) $D$ too small; (lower left) $D$ too large; (lower right) $P$ too small. $L$ is the inter-bead spacing: $L=P-D$.

\subsubsection{Spectral Implications of Test Function Parameters}

A sample scan line of experimental data and its corresponding optimal test function are shown in Figure 4-3. Qualitatively, the fit for both the bead diameter and the bead spacing seems reasonable and the basic size as well as the separation seem to be faithfully captured by the SET.

The sensitivity of the optimization technique to changes in $D$ and $P$ is illustrated in Figure 4-4, which shows examples of the matching process between the same experimental spectrum and four test functions with varying values of $D$ and $P$. In all cases the experimental spectrum is plotted with a dark line while the test function spectrum is shown with a lighter line. The upper left portion of Figure 4-4 shows the comparison between the two spectra for the optimal choice of $D$ and $P$. The locations of the maximum spectral peak and its subsequent harmonics are controlled by the choice of $P$. However, the role of $D$ is to control the shape of the total spectrum and in particular, the roll-off of the spectrum as the wave number increases. This is illustrated in the upper right and lower left panels of Figure 4-4 in which the effect of a sub-optimal choice of $D$ is explored. Since $P$ is optimal, the location of the maximum is still accurately captured. However, the decay rates of the spectra are not well matched and the test spectra either decay too slowly if the choice of $D$ is too small (upper right panel of Figure 4-4) or too quickly if the choice of $D$ is too large (lower left panel of Figure 4-4). Lastly, the lower right panel of Figure 4-4 illustrates 


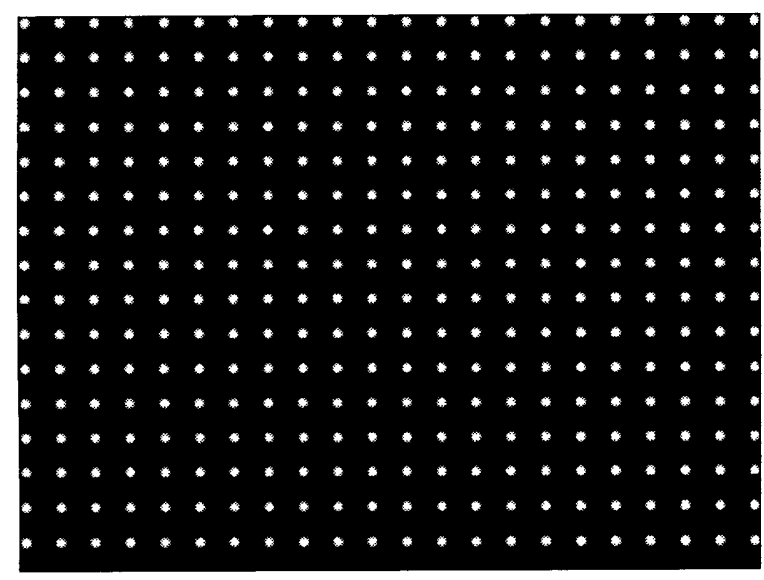

(a) Rectangular Bead Array: $D=10$ pixels, $P=30$ pixels ( $480 \times 640$ pixels $)$.

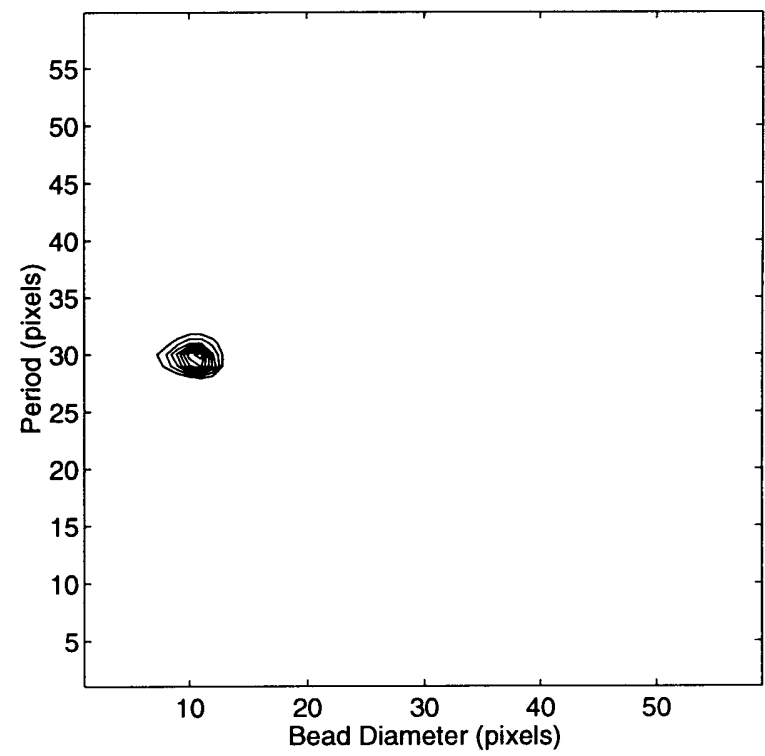

(b) Corresponding Horizontal SET Histogram for Rectangular Bead Array

Figure 4-5: Rectangular Bead Array Test Case

how a non-optimal choice of $P$ leads to a mismatch in the location of the fundamental mode and its higher harmonics.

\subsection{Validation of the SET}

\subsubsection{Rectangular Bead Arrays}

As test cases for the SET algorithm, idealized images containing known bead diameter and bead spacing length scales were analyzed. The SET results were then checked to see that the optimal test function parameters were indeed the prescribed length scales. Figure 4-5 (a) shows a rectangular matrix of beads and Figure 4-5 (b) depicts the corresponding horizontal SET histogram for fixed diameter $D=10$ pixels and period $P=30$ pixels. As can be seen, the corresponding two-dimensional SET histogram has a strong peak at approximately $(D, P)=(10,30)$ pixels. Some smearing of the peak is to be expected since a given scan line will not, in general, pass exactly through the center of a row of beads. Additional uncertainty arises from the fact that the image beads are not identical in mathematical form to the SET test function.

When two greatly disparate bead sizes are present, the SET tends to emphasize the larger bead sizes. This is because the larger beads contain more spectral power and consequently are approximated most effectively by test functions with large bead diameters. While it is possible to create test cases in which the small bead sizes are 


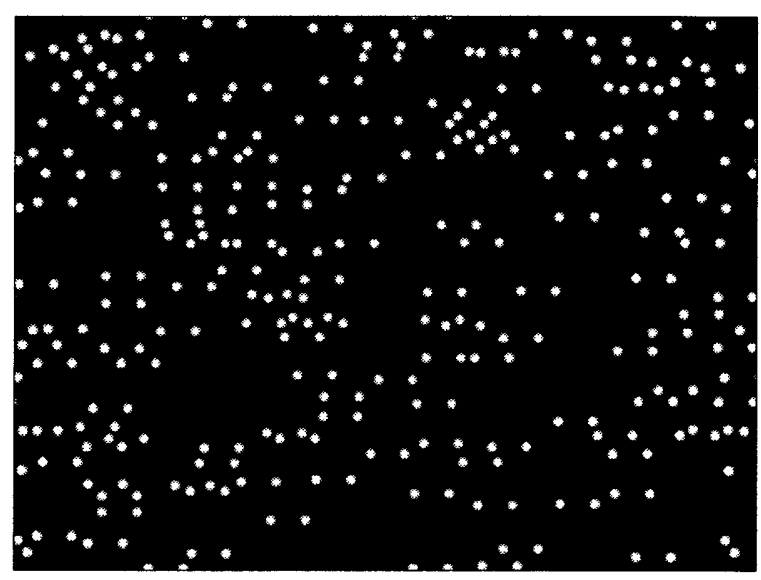

(a) Pseudo-random Bead Array: $D=10$ pixels, $P=30$ pixels (image dimensions: $480 \mathrm{x}$ 640 pixels). Same bead density as rectangular bead array in Figure 4-5

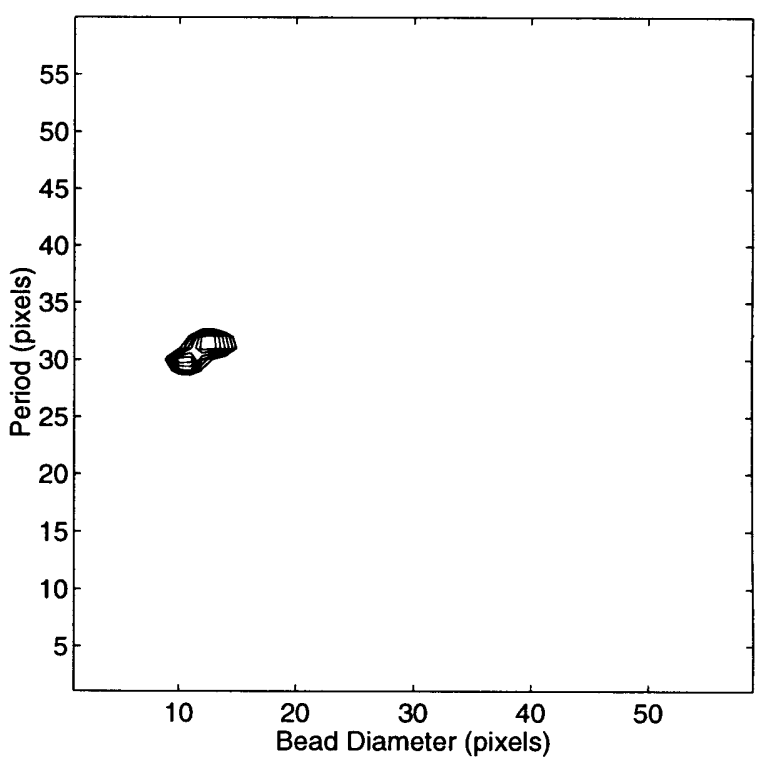

(b) Corresponding Horizontal SET Histogram for Pseudo-random Bead Array

Figure 4-6: Pseudo-Random Bead Array Test Case

ignored in favor of relatively large beads, one can usually detect smaller beads by the presence of secondary peaks in the joint $(D, P)$ SET histogram.

It is possible that the interference of large beads in the detection of smaller beads could be eliminated using a multiple-scale analysis. For example, a multiple-scale scheme might involve sophisticated techniques such as wavelet filtering or a proper orthogonal decomposition to separate the ice features according to size in a preprocessing step before application of the SET. However, after preliminary study, it is not clear that the cost of adding this level of complexity to the SET would be worth the benefit realized since the larger beads offer a reasonable first approximation to the roughness and may even dominate the heat transfer enhancement in the icing problem.

\subsubsection{Pseudo-random Bead Arrays}

Test images more representative of physical ice accretions may be produced by depositing beads of specified diameter and inter-bead spacing in pairs at arbitrary locations in the image until a target bead density is achieved. Figure 4-6 (a) depicts such a pseudo-random array with the same bead density as Figure 4-5 for $(D, P)=(10,30)$ pixels. Figure 4-6 (b) depicts the corresponding SET histogram for the horizontal scan lines. The SET histogram reveals a peak at the expected location of $(D, P) \approx(10,30)$ pixels.

If SET parameters are to be specified in both the horizontal and vertical directions 
within a single image, the test beads can be deposited in triplets with different interbead spacings in either direction. The underlying motivation for this image generation technique is the desire to measure the horizontal and vertical SET parameters from a given experimental image, compute a two-dimensional bead density from a rectangular bead array possessing these parameters, and then produce a pseudo-random bead array with SET parameters similar to the original image.

\subsection{Application of SET to Experimental Data}

\subsubsection{IRT Close-Up Video Data}

The SET histograms for the spanwise (vertical) and streamwise (horizontal) directions for the second frame of the mixed accretion in Figure 2-3 are given in Figures 4-7 (a) and 4-7 (b). For the vertical histogram given in Figure 4-7 (a) the two highest peaks are at $(D, P)$ pairs of $(0.47,0.77) \mathrm{mm}$ and $(0.57,1.16) \mathrm{mm}$ although it is noted that other comparable peaks exist. The two highest peaks in the horizontal histogram (Figure $4-7(\mathrm{~b}))$ are at $(D, P) \approx(0.5,0.83) \mathrm{mm}$ and $(D, P) \approx(0.63,1.06) \mathrm{mm}$. The similarity between the $(0.47,0.77)$ peak in the vertical histogram and the $(0.5,0.83)$ peak in the horizontal histogram as well as the apparent correspondence between the $(0.57,1.16)$ peak in the vertical histogram and the $(0.63,1.06)$ peak in the horizontal histogram suggests a certain degree of isotropy in the accretion image. That is, there is an approximate matching between the horizontal and vertical statistics. Qualitative inspection of the gray-scale image reveals that while it may be difficult to estimate interbead spacings, the roughness elements do appear to have approximately the same dimensions in both the stream and span directions, lending credence to claims of isotropic roughness with respect to bead size. This is reasonable since the image was taken only 10 seconds into the accretion and round roughness elements do not start to become distorted until mechanisms such as streamwise rivulets of surface water dominate at longer times.

\subsubsection{IRT Roughness Castings}

The SET histograms for the spanwise and streamwise directions for the roughness casting of Figure 2-4 are given in Figures 4-8 (a) and 4-8 (b).

There are two dominant structures in the spanwise SET histogram (Figure 4-8 (a)) at $(D, P)$ combinations of $(0.93,1.79) \mathrm{mm}$ and $(0.79,3.57) \mathrm{mm}$. Consideration of the streamwise SET histogram (Figure 4-8 (b)) reveals a dominant peak at $(1.14,3.93) \mathrm{mm}$. Numerically, the second largest structure is at $(0.89,1.68) \mathrm{mm}$ although the presence of the peak is not as pronounced as its counterpart in the spanwise SET histogram at $(0.93,1.79)$. 


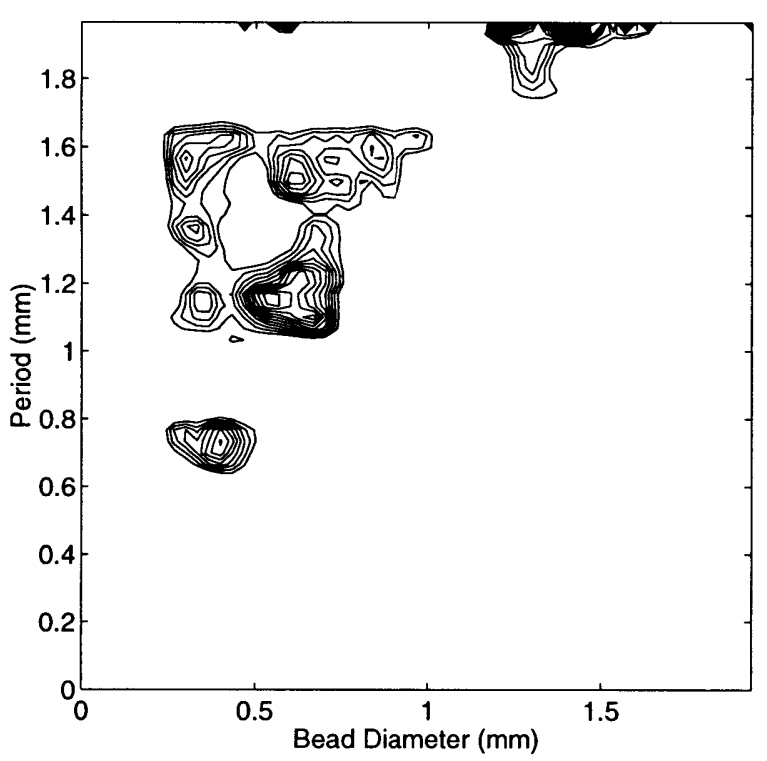

(a) SET histograms for second frame of Figure 2-3: spanwise (vertical) SET

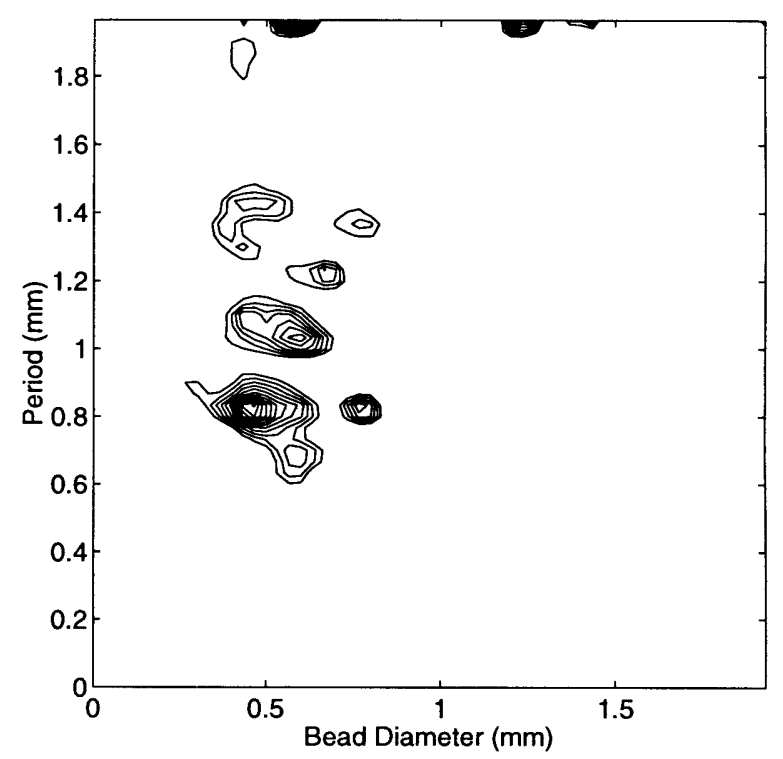

(b) SET histograms for second frame of Figure 2-3: streamwise (horizontal) SET

Figure 4-7: SET Histograms for Mixed Accretion

\subsection{Discussion}

The image generation technique presented above uses a somewhat limited two-dimensional test function in the sense that the roughness element diameters in both the horizontal and vertical directions are assumed to be comparable. That is, this model applies to images that are composed primarily of bead-like elements rather than structures that are elongated in a particular direction. This assumption is more likely to be valid for early stage icing roughness which is typically composed of localized ice growths. The ice growths increase in density and merge at longer accretion times to form complicated, interconnected patterns.

In light of this, one might expect the bead model to more accurately characterize the early IRT video images which were taken during the first 20 seconds of exposure to cloud conditions rather than the casting images which resulted from exposure times an order of magnitude greater. In fact, the SET histograms for the IRT video data do contain much less noise than their casting image counterparts (see Figures 4-7 (a), 4-7 (b) and Figures 4-8 (a), 4-8 (b)), implying that the test function was a better "fit" for the IRT images. Also, qualitative comparison of the IRT and casting images shows that while there does seem to be characteristic length scales present in both images, the casting is significantly more complicated and possesses interconnected elements that deviate from idealized bead-like roughness due to the more advanced stage in the accretion when the casting was taken.

This suggests that the bead model is more appropriate for the early stage roughness. However, this is not really a limitation since, as previously discussed, it is the 


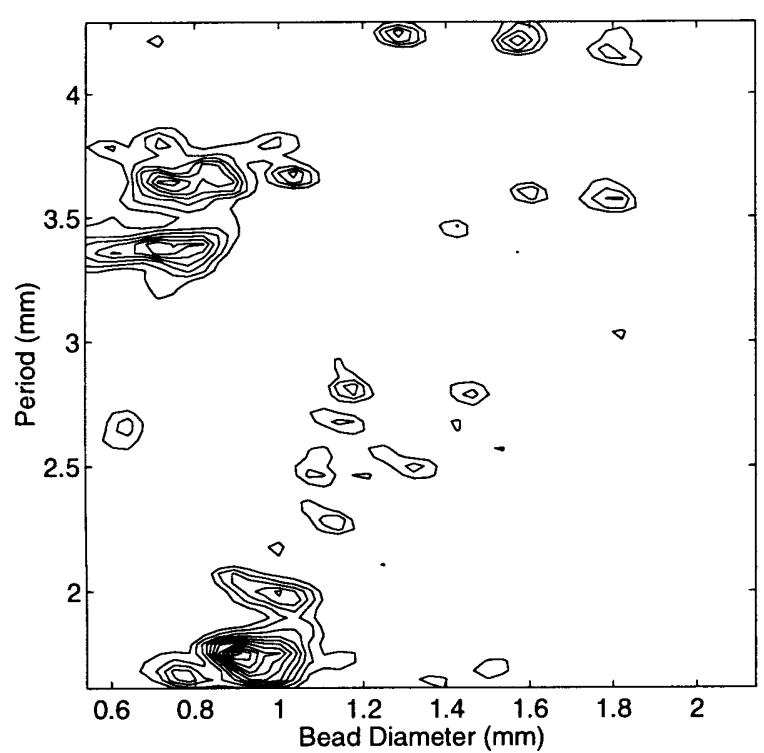

(a) SET histogram for casting image of Figure 2-4: spanwise (horizontal) SET

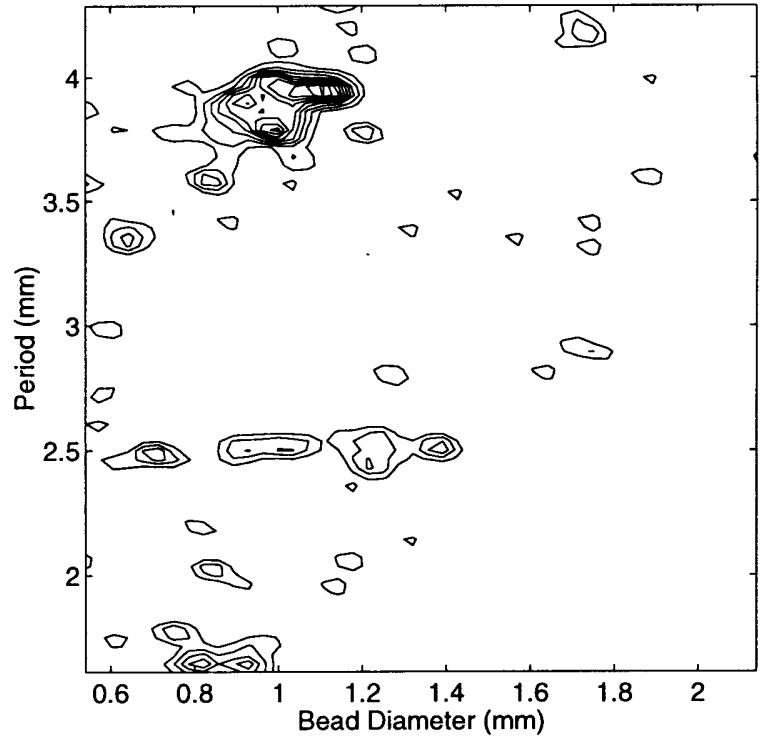

(b) SET histogram for casting image of Figure 2-4: streamwise (vertical) SET

Figure 4-8: SET Histograms for Icing Casting

role of early stage roughness in enhancement of heat transfer and boundary layer transition that is primarily of interest. 


\section{Chapter 5}

\section{Simulated Roughness for Dry Wind Tunnel Testing}

The next step in completing the methodology shown in Figure 1-1 for connecting experimental icing data to the heat transfer models used in ice accretion codes is to construct physical roughness samples that have the same SET parameters as the experimentally observed ice accretions. Once this is accomplished, the methodology is realized by conducting dry wind tunnel tests on the physical roughness samples to evaluate the impact on convective heat transfer.

\subsection{Use of the SET for Roughness Construction}

One of the strengths of the SET is that the parameters produced by the method have clear physical interpretations. This is an important aspect of the analysis since such a scheme is easily translated into a production technique for physical roughness models. As previously mentioned, the SET is a primitive object placement rule technique; simulated textures are composed of well-defined primitive objects at possibly different scalings which are inserted into a texture field according to some placement rule. For the SET, the primitive objects are beads and the placement rule is composed of bead spacing selection, bead overlap prohibition, and a bead number density criterion.

In contrast, stochastic texture analysis techniques which utilize parameters such as fractal dimension and Markov random field clustering constants[14] do not always have clear physical meaning in the context of physical roughness production. Consequently, the efficacy of these characterizations of icing roughness as a guide to experimental roughness construction is questionable. Alternatively, the obvious extension of the SET to a construction scheme for physical roughness samples is an automated bead deposition technique. One such scheme is described below.

\subsection{Hardware}

The technique for constructing physical roughness samples is epoxy bead deposition using an automated syringe. The apparatus used to deposit epoxy beads (Figures 5-1 


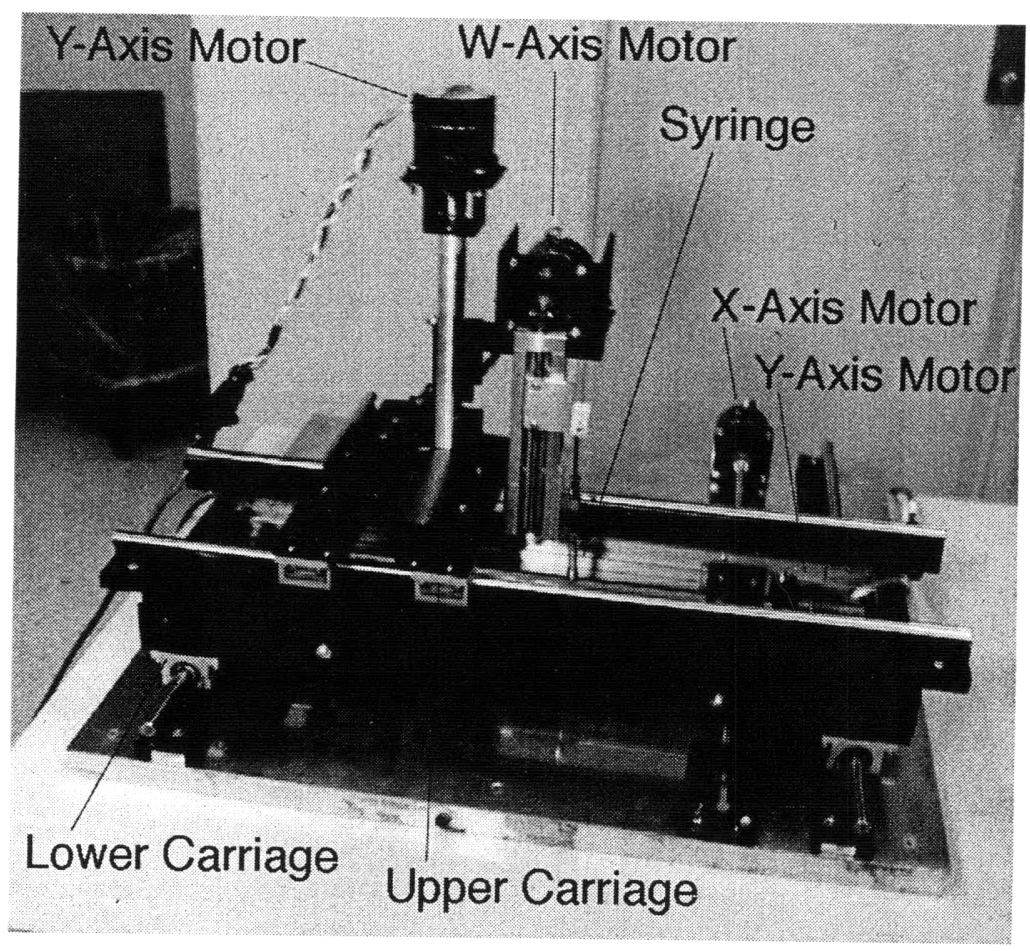

Figure 5-1: Three-dimensional traverse for automated epoxy bead deposition. X, Y and $\mathrm{Z}$ axes control syringe position while $\mathrm{W}$ axis controls the syringe plunger.

and 5-2) consists of a three-dimensional computer-controlled traverse which positions a precision micro-liter syringe for delivery of a soft, slightly thixotropic, two-part epoxy. The traverse positions the syringe to an accuracy of $0.007 \mathrm{~mm}$ in three dimensions over a substrate area of $28 \mathrm{~cm} \times 28 \mathrm{~cm}$. A fourth computer-controlled axis actuates the plunger on the syringe to dispense set volumes of epoxy.

The epoxy selected provided a working time in excess of 2 hours and was of thin consistency but could be thickened by pre-curing to provide larger contact angles between the droplet and the substrate. The needle sizes used ranged between 26 gauge and 18 gauge which produced droplet sizes from $0.4 \mathrm{~mm}$ to over $2 \mathrm{~mm}$. This range of bead sizes was consistent with the length scales observed in the IRT video images as well as the casting images. Blunt, $90^{\circ}$ bevel needles were used so that the epoxy on the tip of the needle could be touched to the substrate and the bead inflated to the desired volume. This prevented excess epoxy from clinging to the needle tip and resulting in irregular bead volumes on successive applications.

The substrates used were polished aluminum and Plexiglas. In order to provide reasonable bead contact angles, the substrates were treated with wax or a combination of wax and paint. To control the bead heights, one could control the volume of epoxy delivered, thicken the epoxy with pre-curing, or "build up" individual beads with multiple applications of epoxy between partial curings. Additional techniques useful for control of bead geometry include adjustment of the substrate temperature and curing the bead arrays up-side-down to prevent flattening of the beads under the influence of gravity. For a bead diameter of $2 \mathrm{~mm}$, obtainable bead heights ranged 


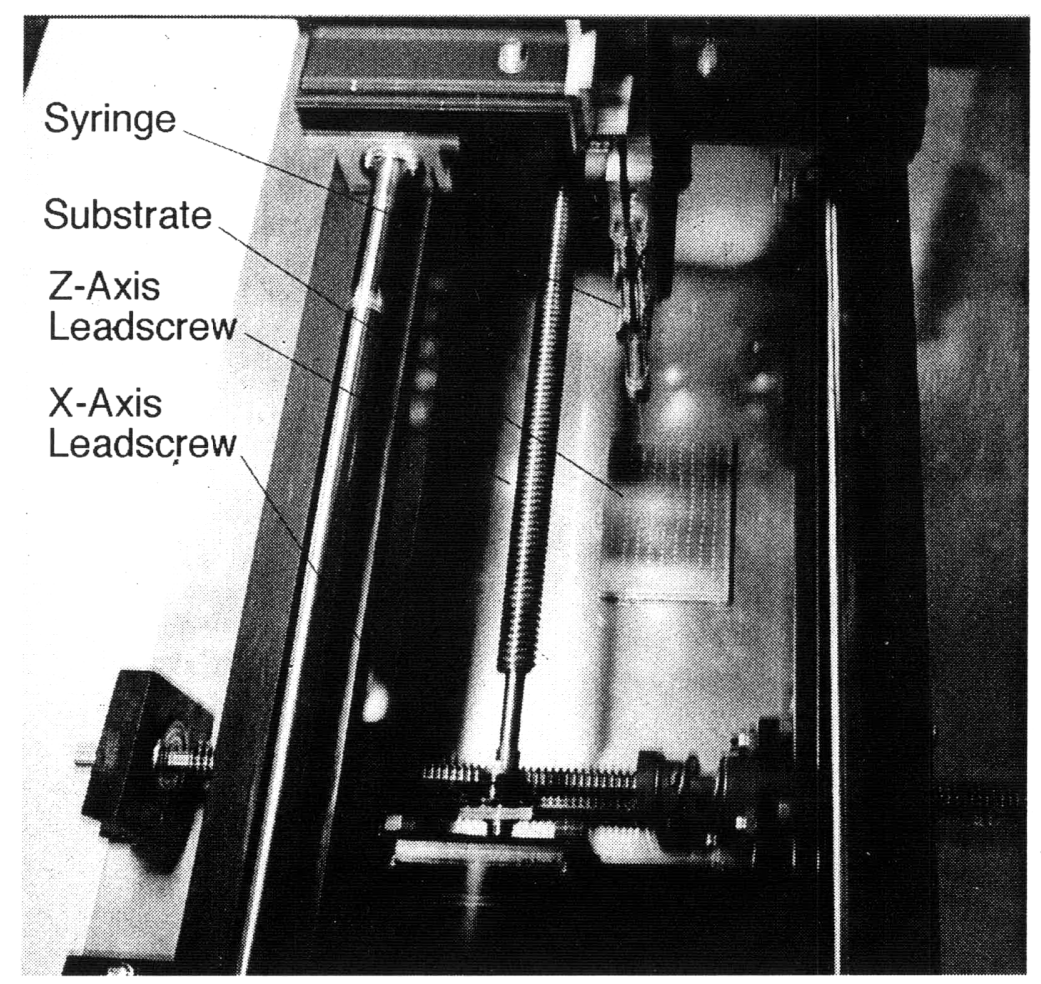

Figure 5-2: Three-dimensional traverse for automated epoxy bead deposition. The $\mathrm{W}$ axis and syringe are clearly visible as well as the substrate and epoxy beads.

from $0.2 \mathrm{~mm}$ to $0.75 \mathrm{~mm}$.

\subsection{Syringe Calibration with the SET}

To calibrate the syringe/epoxy assembly, an array of test beads was deposited for different syringe plunger displacements. This calibration array is shown in Figure 53 and is analogous to the rectangular bead arrays of the type shown in Figure 45. The SET was then used to analyze the various scan lines for each bead size in the calibration image. Bead diameters from the SET histograms for each plunger displacement were noted and plotted (Figure 5-4). It should be noted that if factors such as the needle size, epoxy consistency, or substrate treatment are altered, a new calibration curve must be constructed.

\subsection{Modeling of Casting Data}

As previously discussed, the casting images are less than ideal for SET analysis due to the long accretion times which bring into question the validity of the isolated bead array model implicit in the SET. However, the castings do provide the opportunity for wind tunnel tests that directly compare enhanced heat transfer on the SET-based roughness to enhanced heat transfer on the real icing roughness (as represented by 


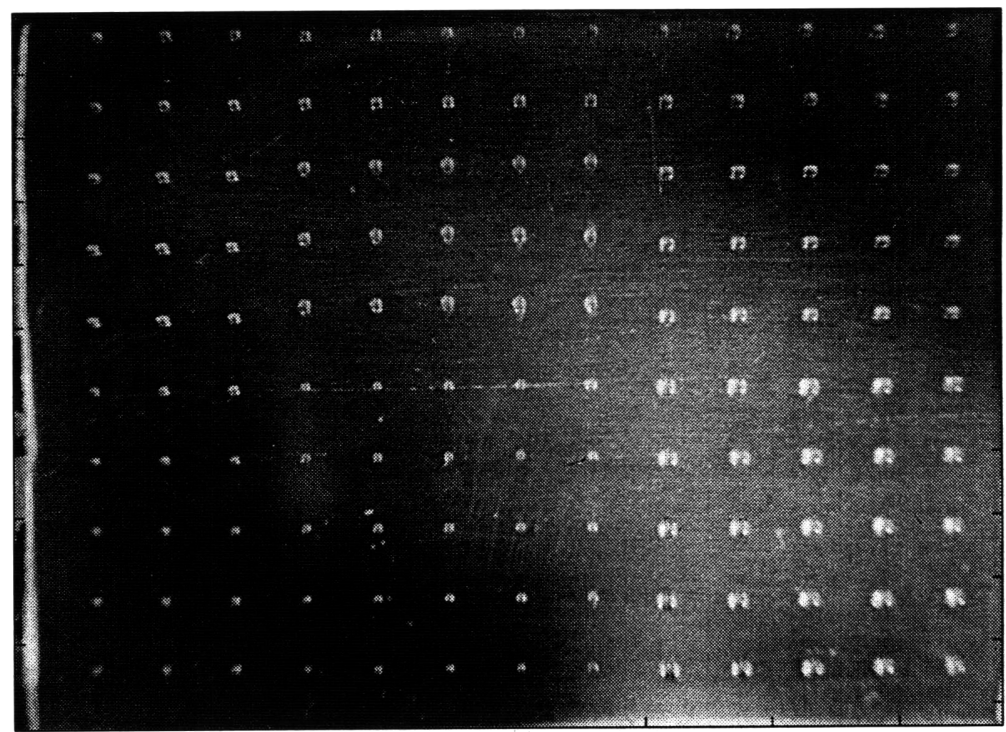

Figure 5-3: Epoxy bead array for SET-based calibration of syringe. Horizontal field of view is $7 \mathrm{~cm}$. Various syringe plunger displacements are used to produce beads of different sizes $(0.4 \mathrm{~mm}-1.4 \mathrm{~mm})$.

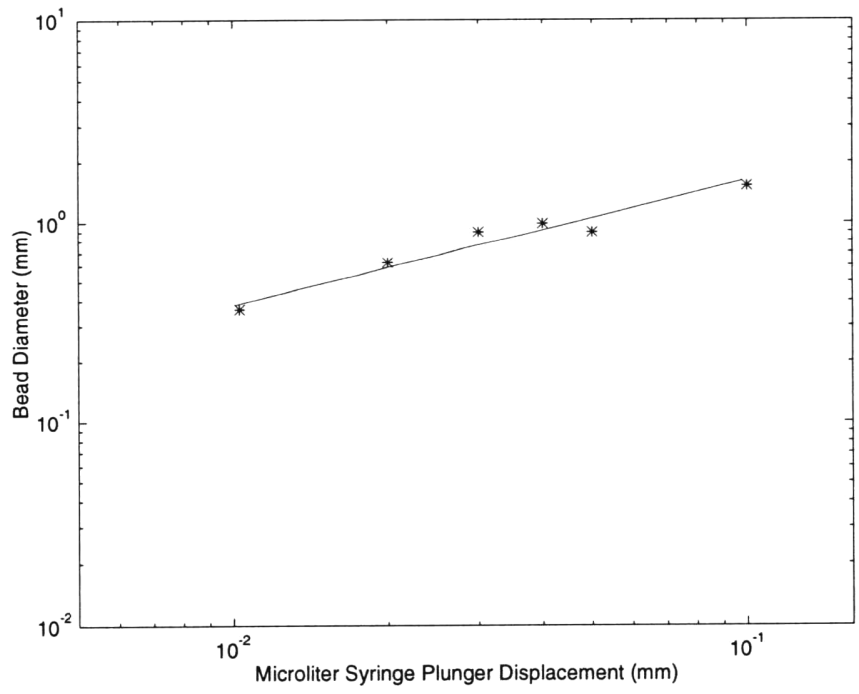

Figure 5-4: SET-based calibration curve for syringe/epoxy assembly. 


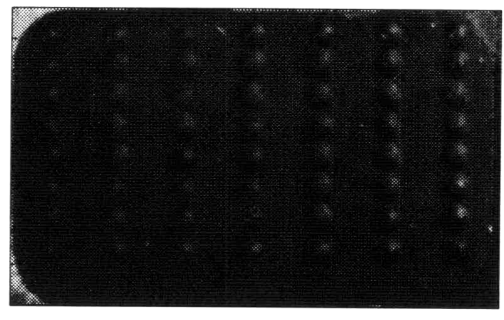

(a) Rectangular Epoxy Bead Array

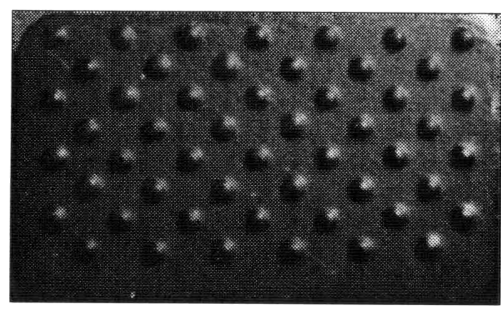

(b) Staggered Rectangular Epoxy Bead Array

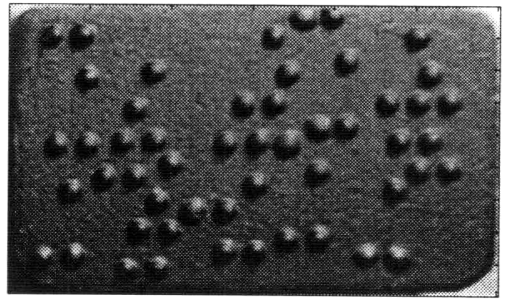

(c) Pseudo-Random Epoxy Bead Array

Figure 5-5: Experimental Epoxy Bead Arrays $(17 \mathrm{~mm} \times 28 \mathrm{~mm})$

the castings).

The first step in the process is to photograph the castings and perform the SET analysis. The casting used is that shown in Figure 2-4. The SET histograms for the spanwise and streamwise directions are given in Figures 4-8 (a) and 4-8 (b). The dominant $(D, P)$ combination in the spanwise SET histogram at $(0.93,1.79) \mathrm{mm}$ and the corresponding streamwise peak at $(0.89,1.68) \mathrm{mm}$ were selected as a first approximation to modeling the casting roughness.

Next, the roughness arrays are constructed using the epoxy bead deposition technique described above. The roughness array configurations selected for this study are shown in Figures 5-5 (a), 5-5 (b) and 5-5 (c). The bead density for the pseudorandom array was equal to that of the rectangular array. A staggered array whose adjacent rows are $180^{\circ}$ out of phase was also investigated.

The SET analysis is then applied to photos of the epoxy bead arrays to check that the SET parameters are those prescribed by the analysis of the casting image.

\subsection{Wind Tunnel Testing}

As the final step in correlating the modeled roughness to the experimental icing roughness, comparative heat transfer studies are conducted in a dry wind tunnel on the epoxy bead arrays and the experimentally-obtained casting. The roughness plugs of Figures 5-5 (a), 5-5 (b), and 5-5 (c) have been tested by Torres[19] using techniques similar to those used by Henry et al.[4].

Preliminary results seem to confirm the conclusions of Henry et al. In particular, the bead size and spacing parameters of the SET appear to capture the same "packing effects" of uniform enhancement of heat transfer for dense bead arrays and localized enhancement of heat transfer for more widely spaced arrays. 


\section{Chapter 6}

\section{Conclusions}

The major goal of this work, providing useful alternatives to existing roughness characterization (most notably, ESGR), has been met with encouraging results. Two very distinct methods for quantitative analysis of ice accretion images have been investigated. The spectral estimation technique (SET) is versatile and appears to be extremely sensitive at picking out physically relevant characteristics observed in ice accretions. In addition, the MRF model has the capability of generating synthetic but realistic-looking ice images based upon experimentally observed ice accretions. However, lack of a physical interpretation of the clustering parameters, as well as difficulty in estimating them, ruled the MRF infeasible since there was no simple extension of the technique to the construction of physical roughness samples.

The SET is particularly promising since it provides a reliable method for extracting physical information from accretion images. Its complexity is only limited by the choice of the test function, and one can easily imagine more sophisticated roughness models being implemented. For example, accretion images often show a range of bead sizes. To reflect this, the SET test function might be defined with two bead sizes and two bead spacings whose characteristic values were extracted from the IRT video data. Equivalently, the second-best-fit test function could be used as an approximation of a second scale in the image.

SET analysis with epoxy bead deposition is a feasible method for connecting IRT images to heat transfer enhancement on surface roughness. However, since the data from the heat transfer tests of Torres [19] are preliminary, a thorough analysis of the IR thermographs has yet to be completed. These results must then be incorporated into an improved heat transfer model for LEWICE so that predicted ice shapes can be compared to the experimentally observed ice shapes in the IRT tests. More dry heat transfer testing will be required to determine how many bead sizes from the experimental SET histograms must be retained for an accurate representation of the roughness.

In the long term, more IRT experiments are needed to develop a data set of early icing roughness over a range of cloud conditions so that more heat transfer studies can be conducted. Ideally, the SET would be applied to a direct representation of the ice surface topography rather than to uncalibrated video images. For example, the roughness data might be in the form of profilometer surveys or stereo video images. 
This improvement in data acquisition would eliminate much of the ambiguity inherent in ordinary video images by providing roughness height information - a key element for comparing enhanced heat transfer on real-world accretions to that observed on SET-designed roughness.

In the context of a larger aircraft icing problem, modeling the complex geometries that result from ice formation on aerodynamic surfaces is a difficult but necessary business. Understanding what types of initial roughness will arise on wings under glaze flight conditions is critical for predicting how heat transfer will be enhanced on the substrate and, consequently, what global ice shapes will emerge at long time scales. 
Appendix A

\section{Experimental Data and SET Diagrams}


$1 \mathrm{sec}$

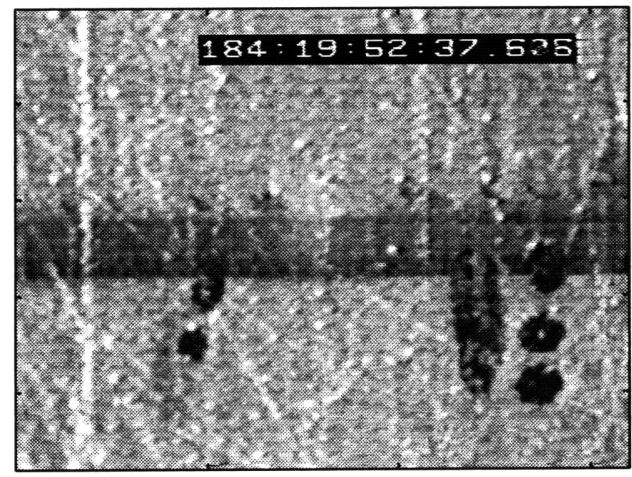

$10 \mathrm{sec}$.

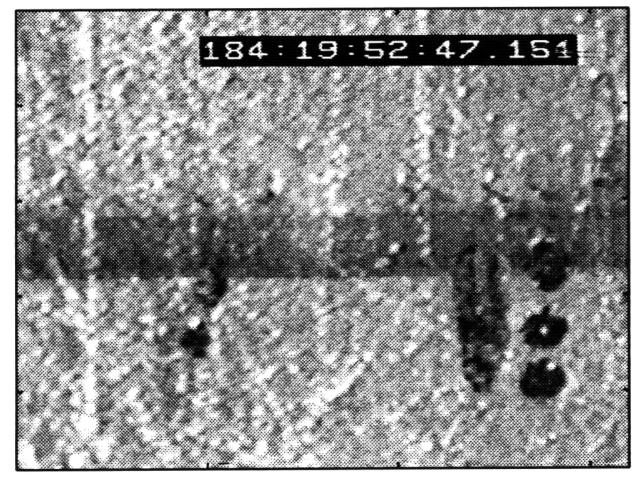

$20 \mathrm{sec}$.

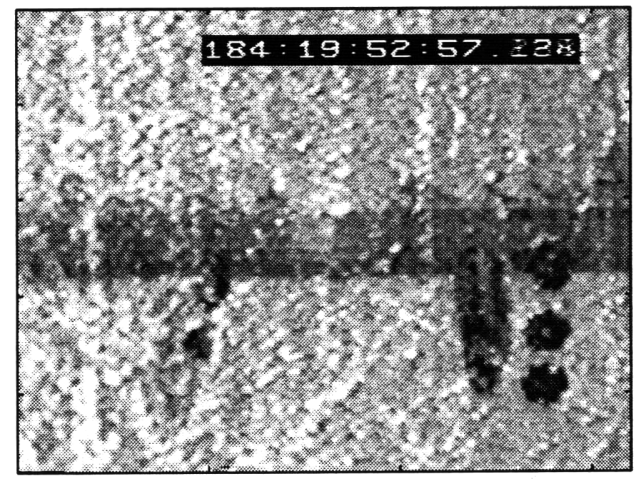

$5 \mathrm{sec}$

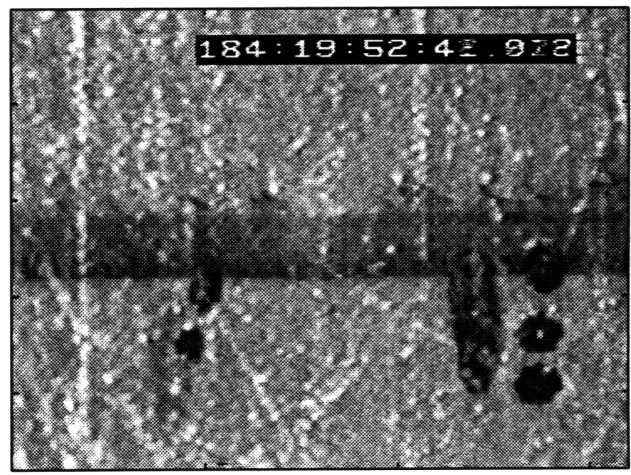

$15 \mathrm{sec}$

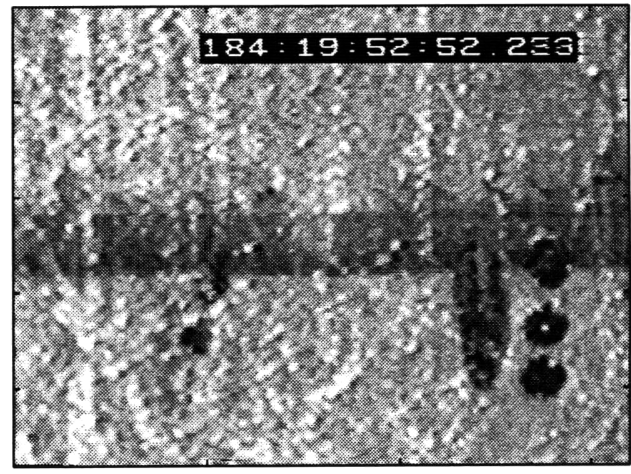

Figure A-1: Grayscale images for mixed accretion T184.19.52. 


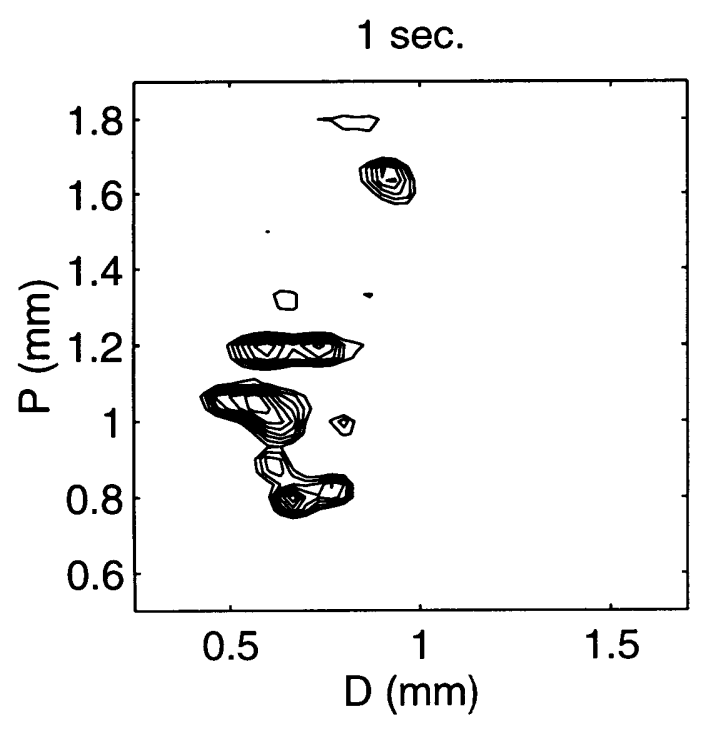

$10 \mathrm{sec}$.

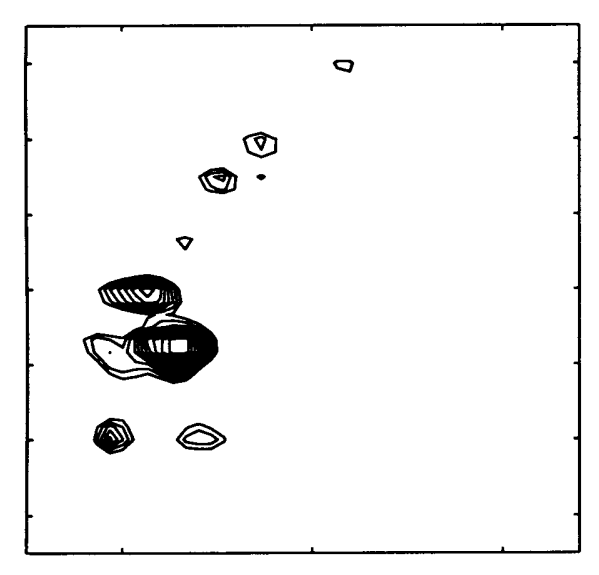

$20 \mathrm{sec}$.

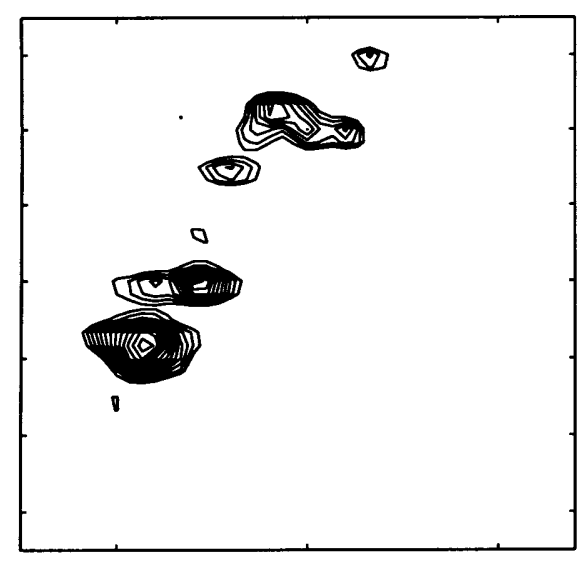

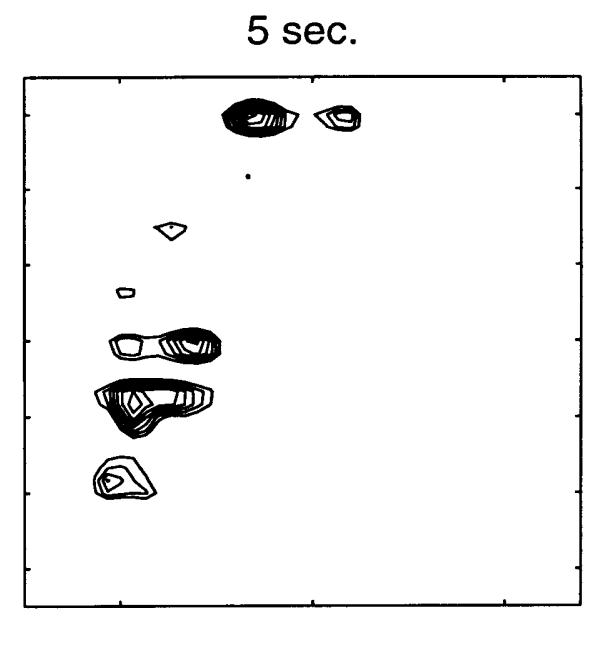

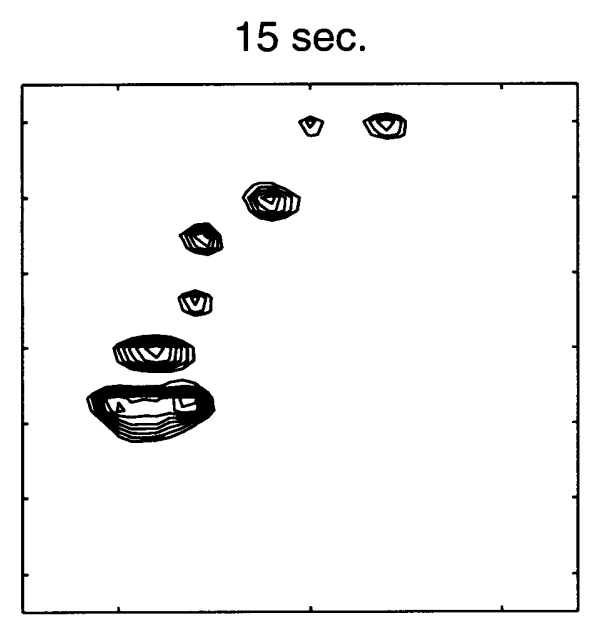

Figure A-2: SET distribution for mixed accretion T184.19.52. 
$1 \mathrm{sec}$.

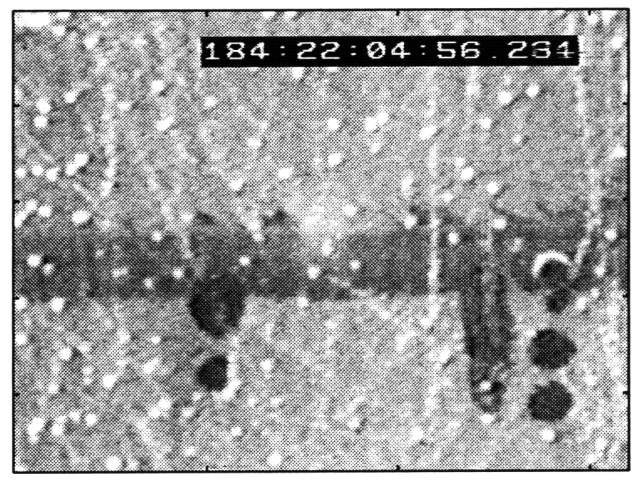

9 sec.

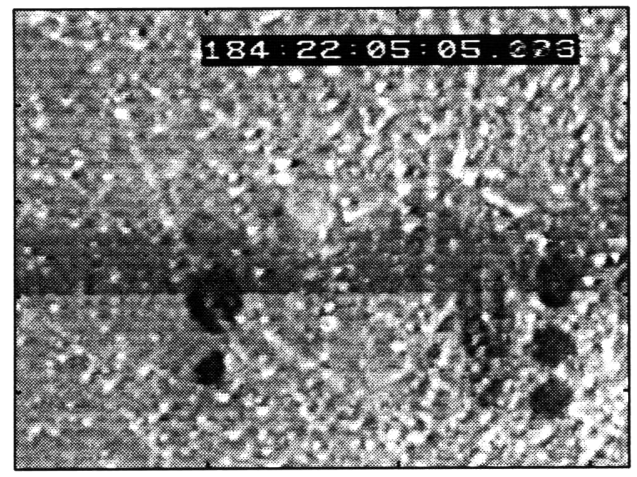

4 sec.

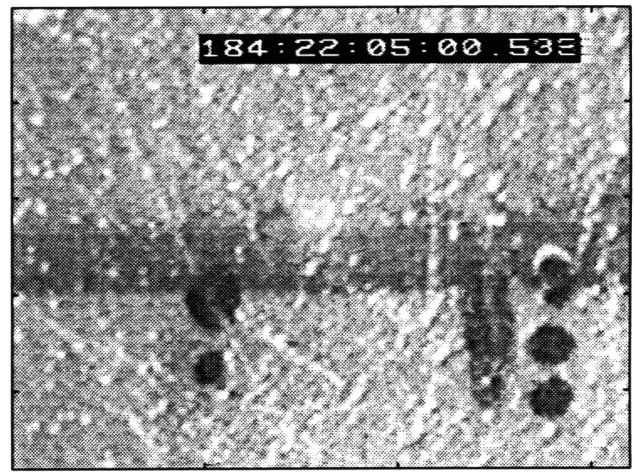

$14 \mathrm{sec}$.

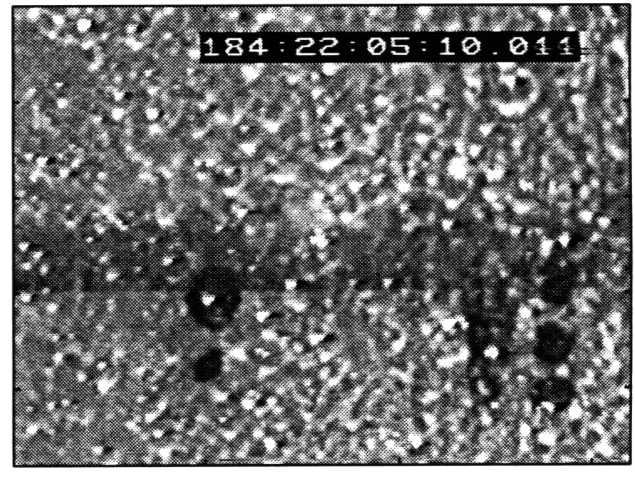

$19 \mathrm{sec}$.

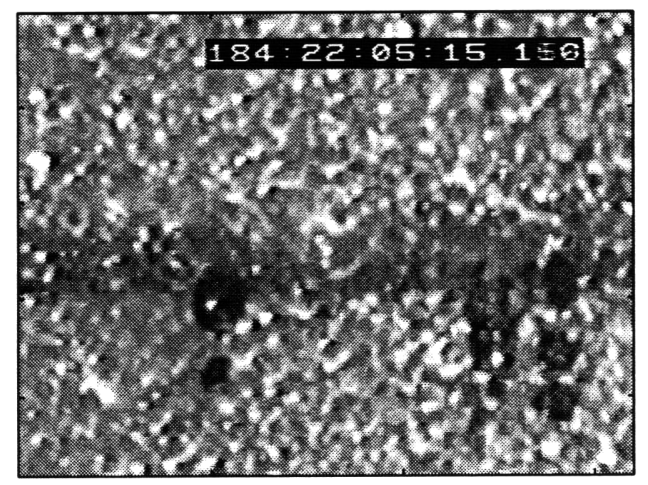

Figure A-3: Grayscale images for glaze accretion T184.22.05. 

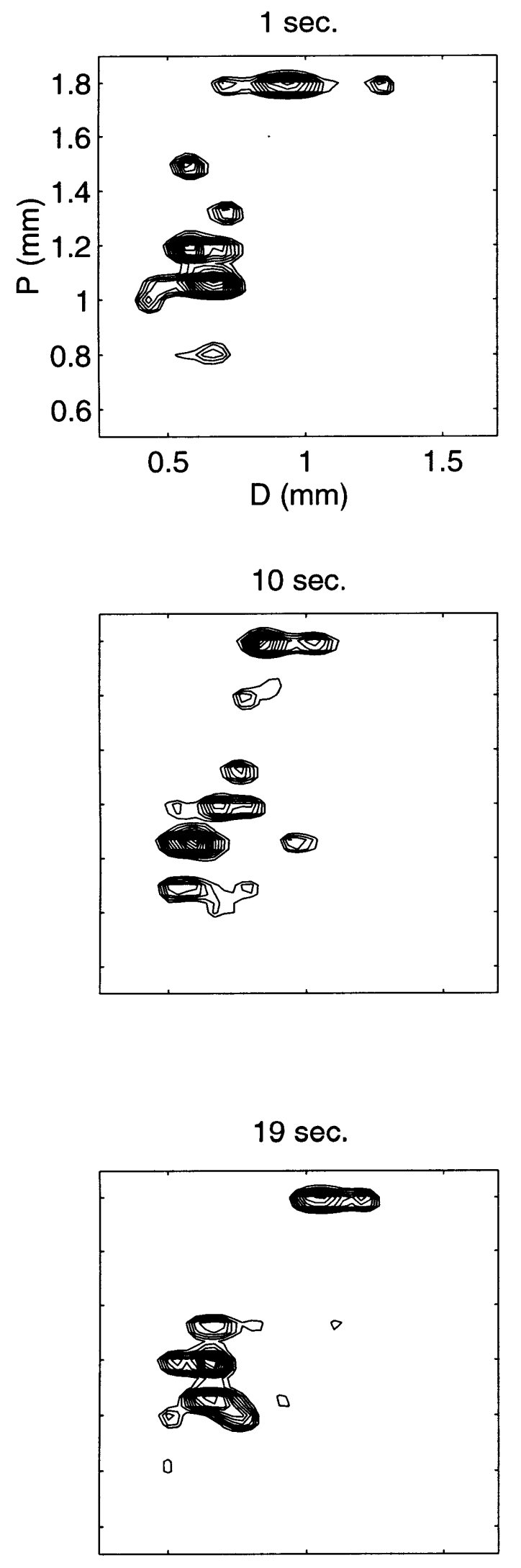
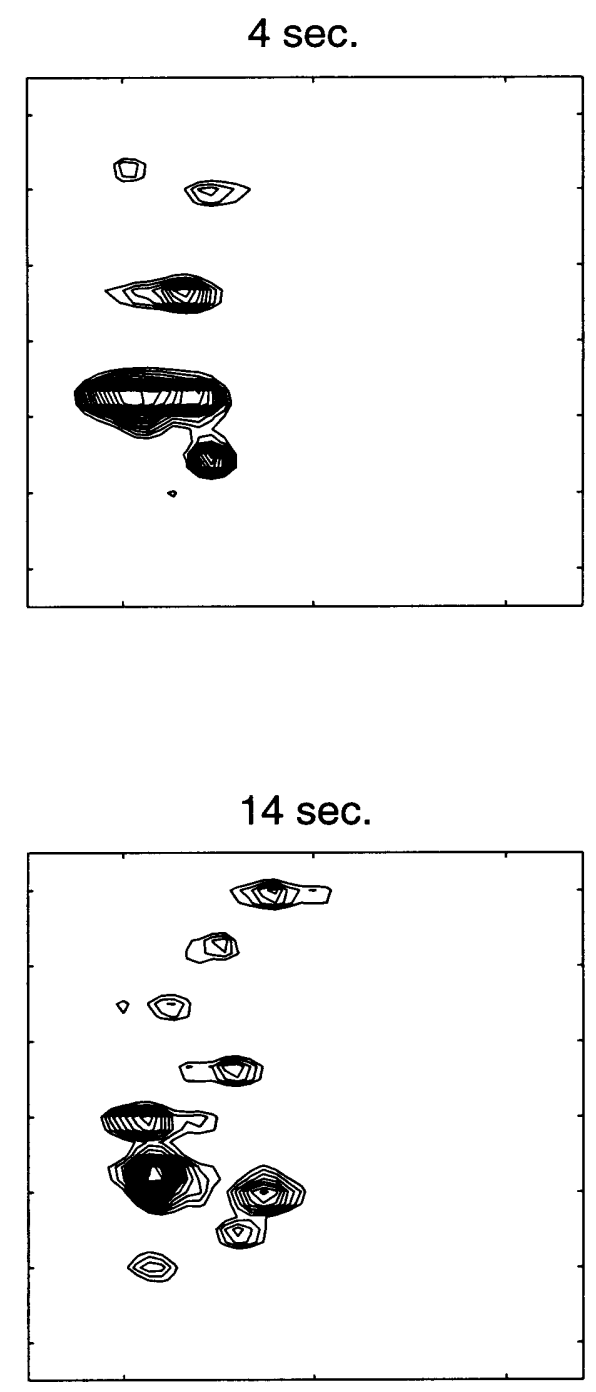

Figure A-4: SET distribution for glaze accretion T184.22.05. 
$1 \mathrm{sec}$.

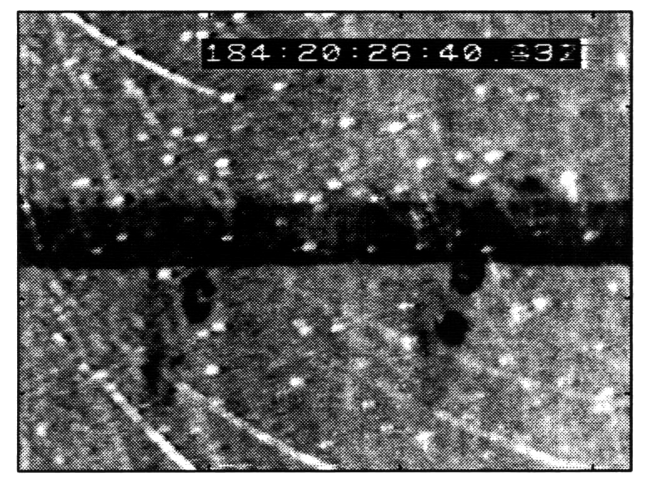

$10 \mathrm{sec}$.

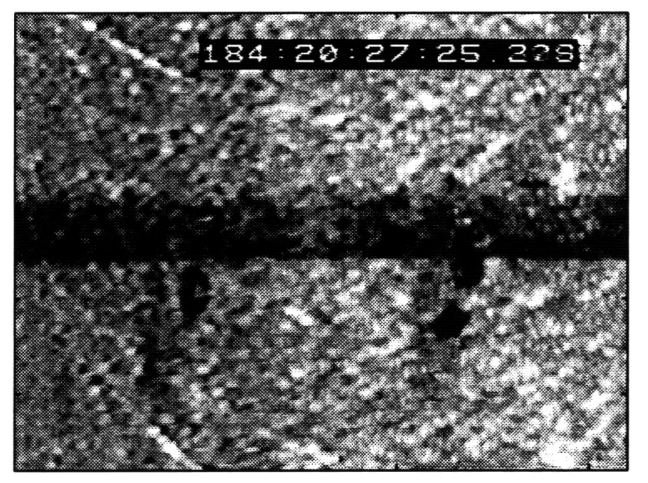

$5 \mathrm{sec}$.

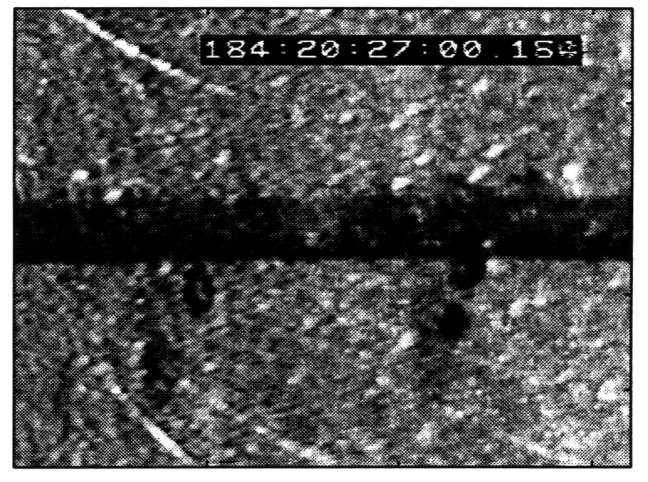

15 sec.

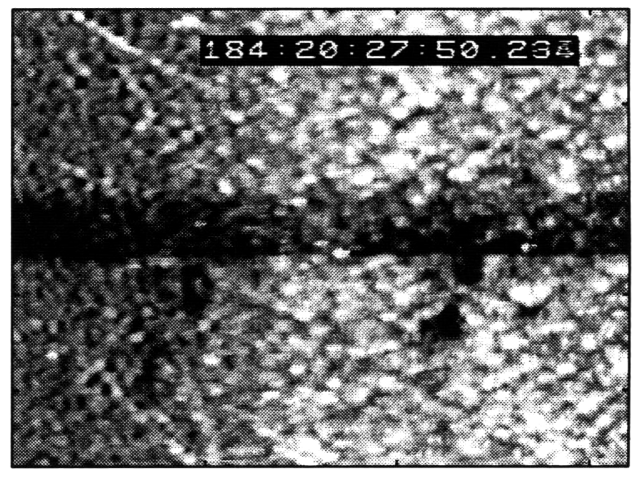

$20 \mathrm{sec}$.

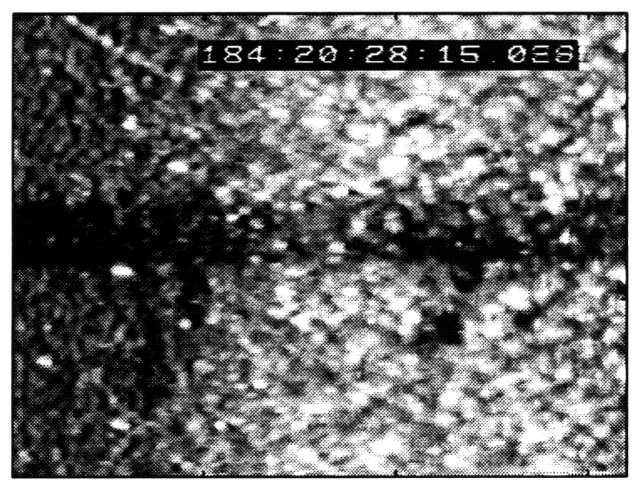

Figure A-5: Grayscale images for mixed accretion T184.20.26. 

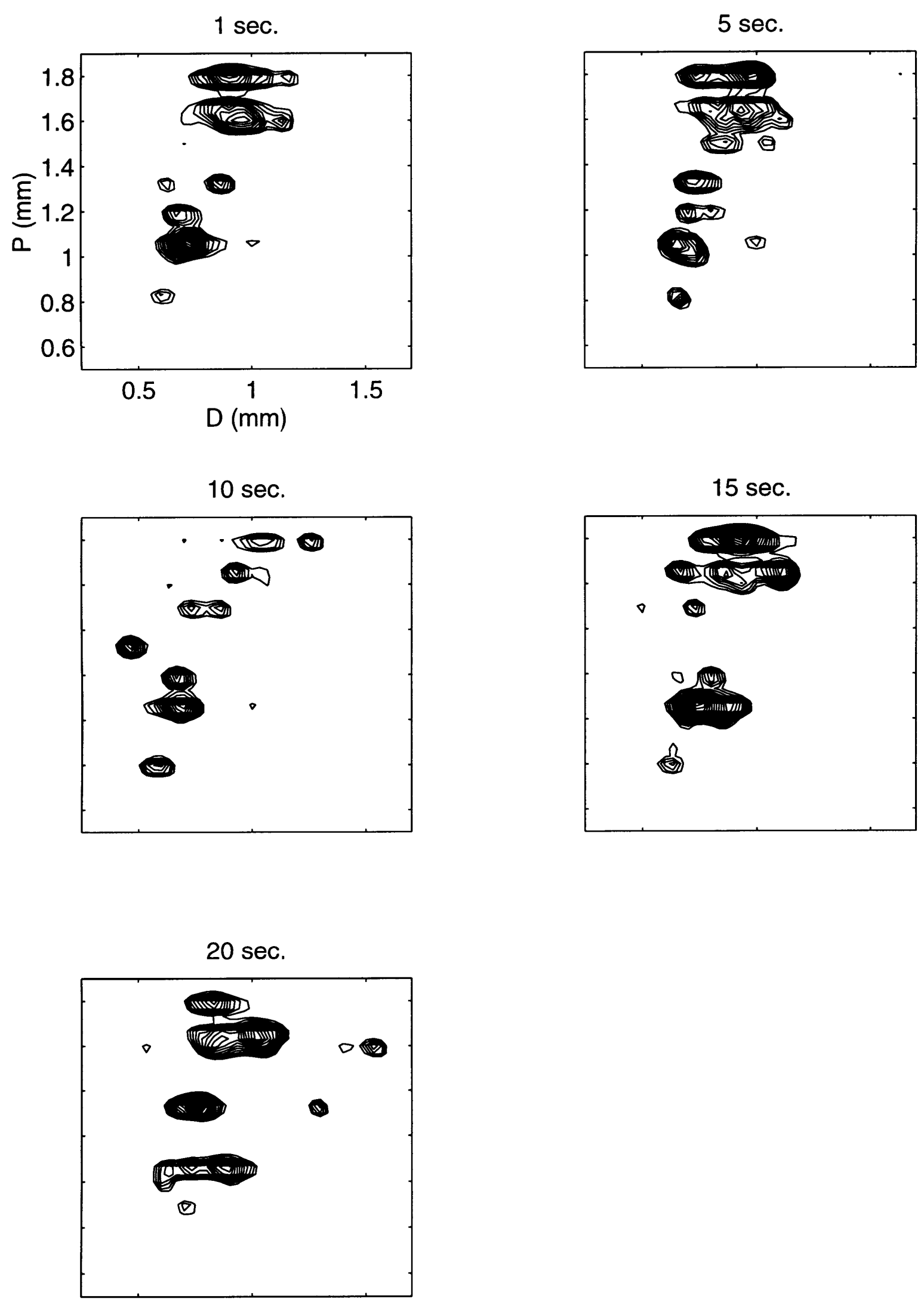

Figure A-6: SET distribution for mixed accretion T184.20.26. 
$1 \mathrm{sec}$.

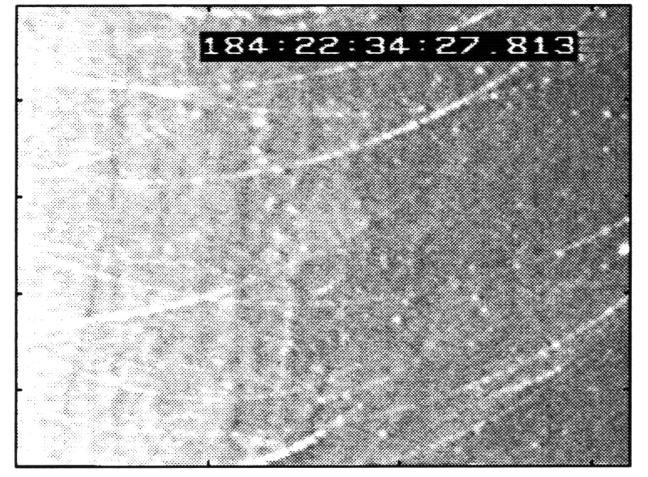

$12 \mathrm{sec}$.

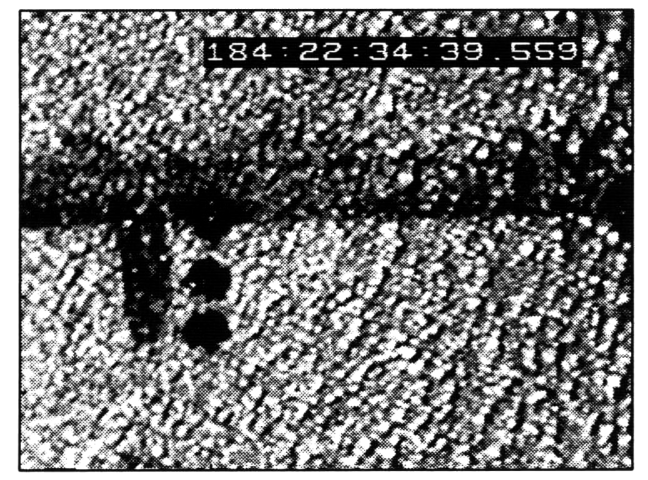

6 sec.

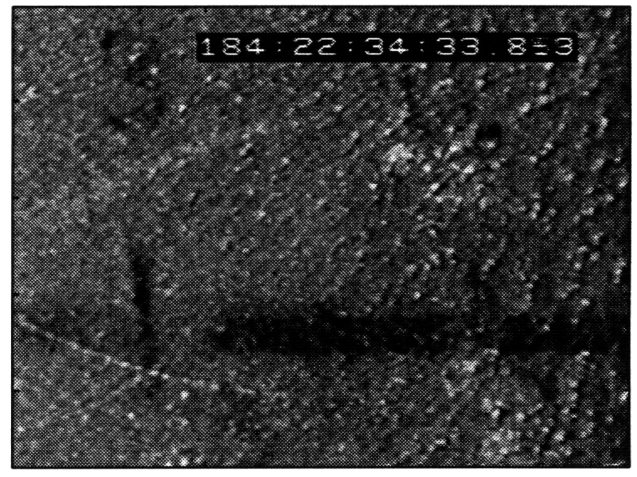

$17 \mathrm{sec}$.

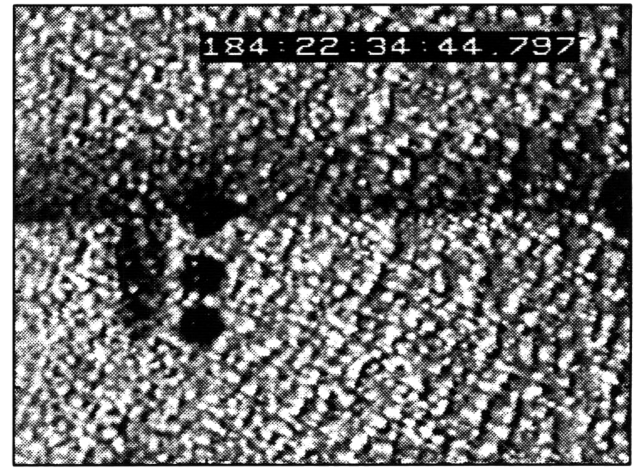

$22 \mathrm{sec}$.

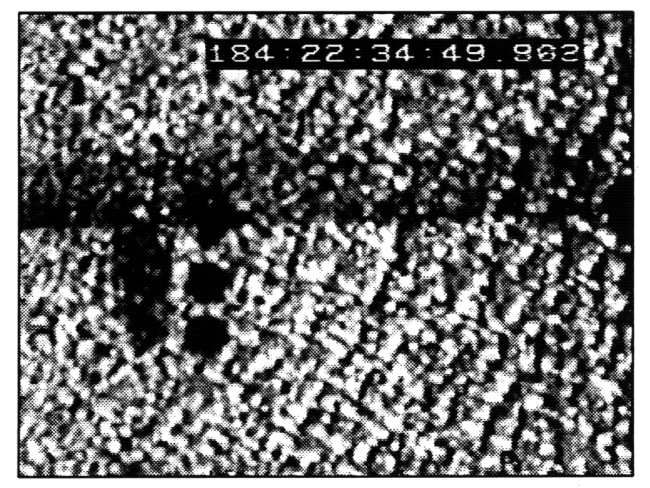

Figure A-7: Grayscale images for mixed accretion T184.22.34. 


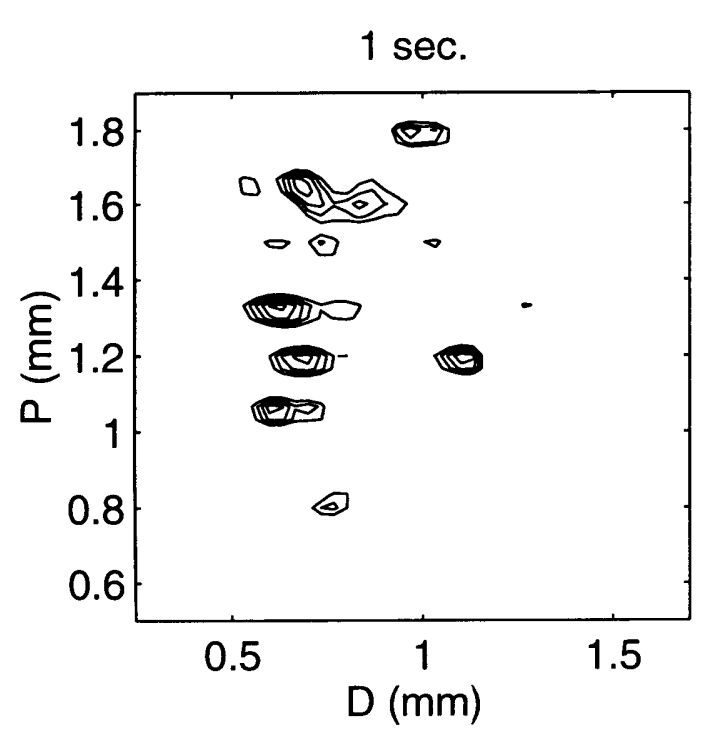

$12 \mathrm{sec}$.

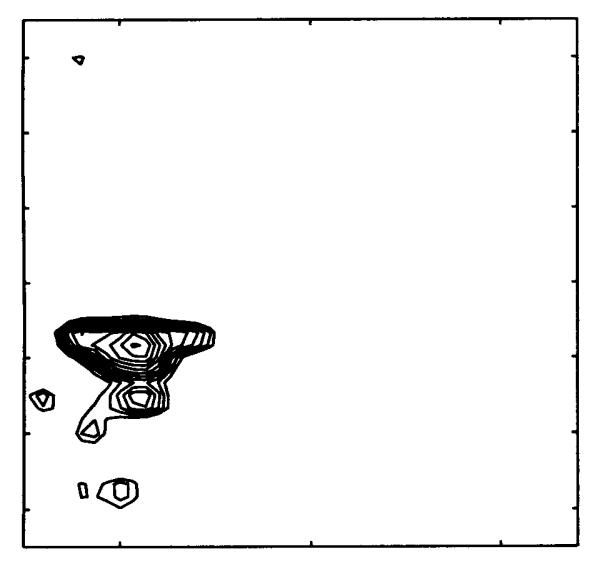

22 sec.

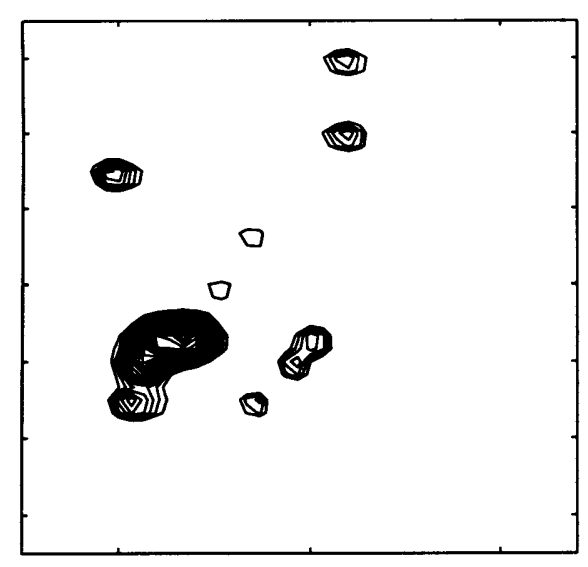

$6 \mathrm{sec}$.

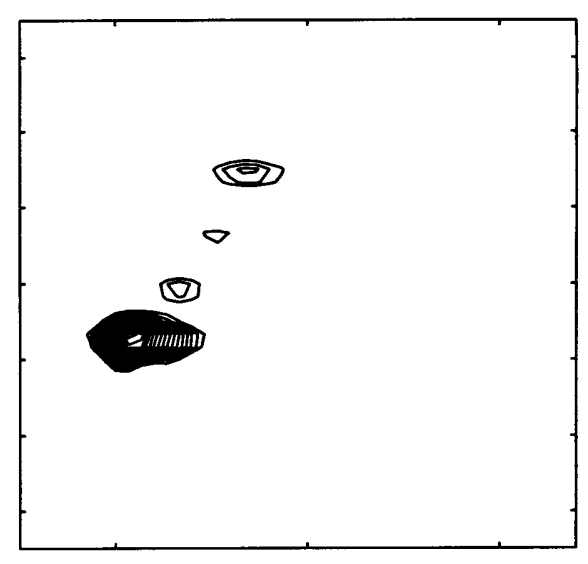

$17 \mathrm{sec}$.

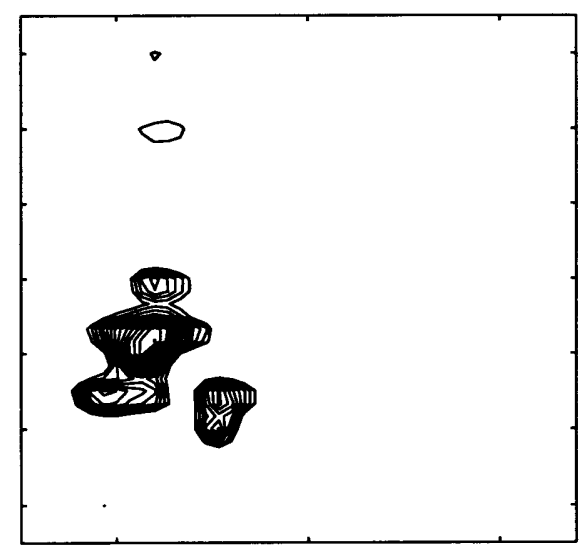

Figure A-8: SET distribution for mixed accretion T184.22.34. 
$1 \mathrm{sec}$

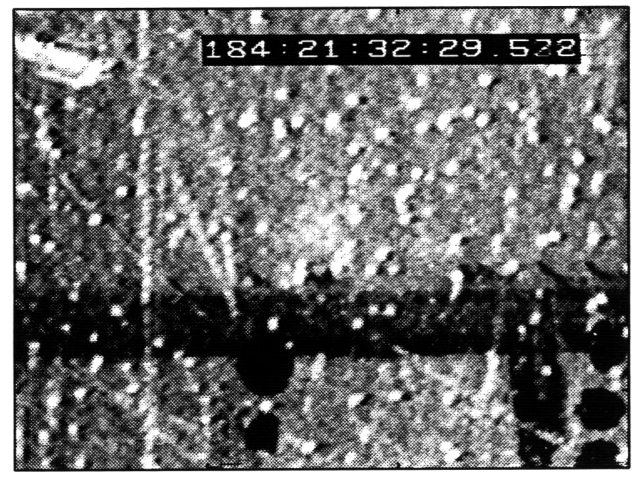

$10 \mathrm{sec}$.

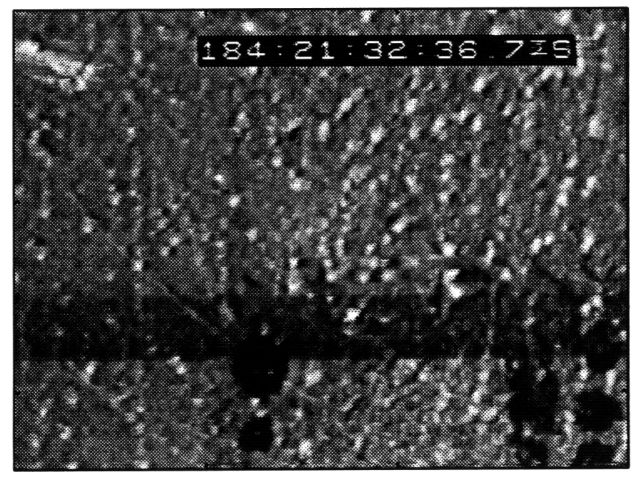

$6 \mathrm{sec}$

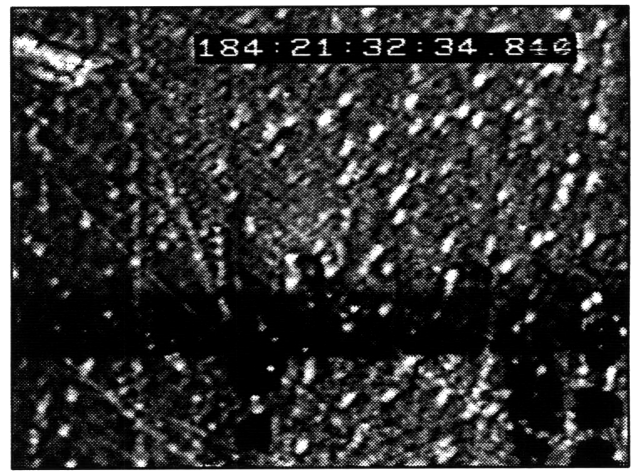

$16 \mathrm{sec}$.

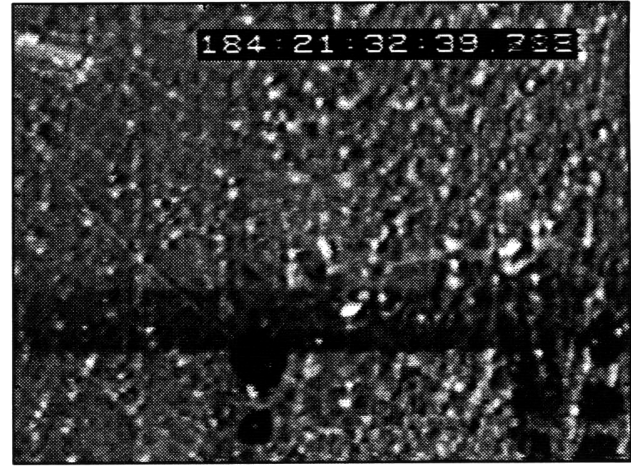

$20 \mathrm{sec}$.

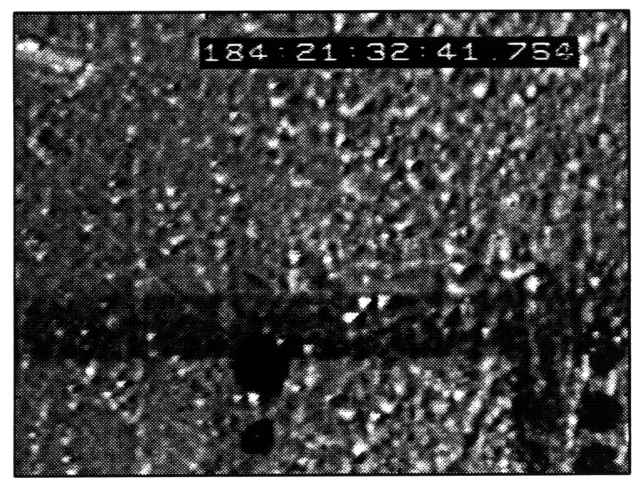

Figure A-9: Grayscale images for glaze accretion T184.21.32. 


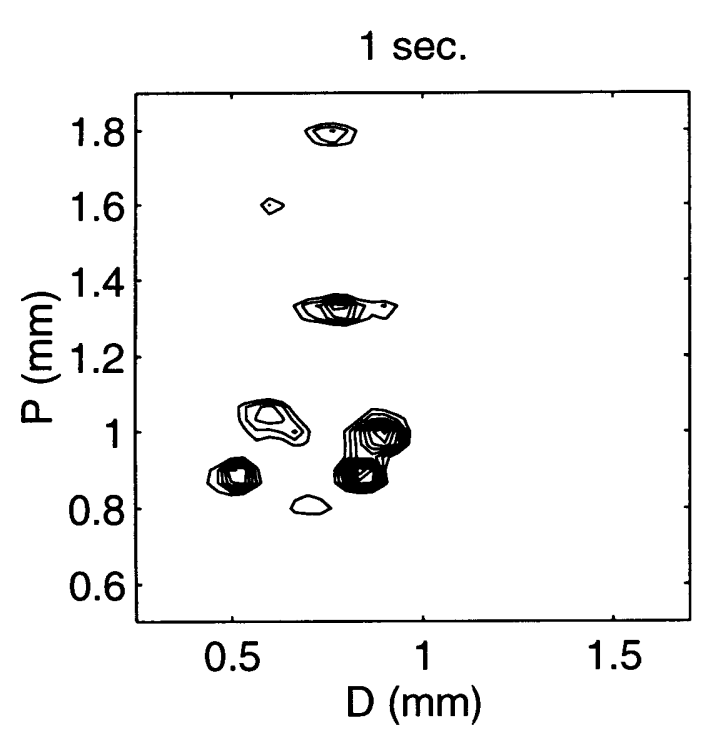

$10 \mathrm{sec}$.

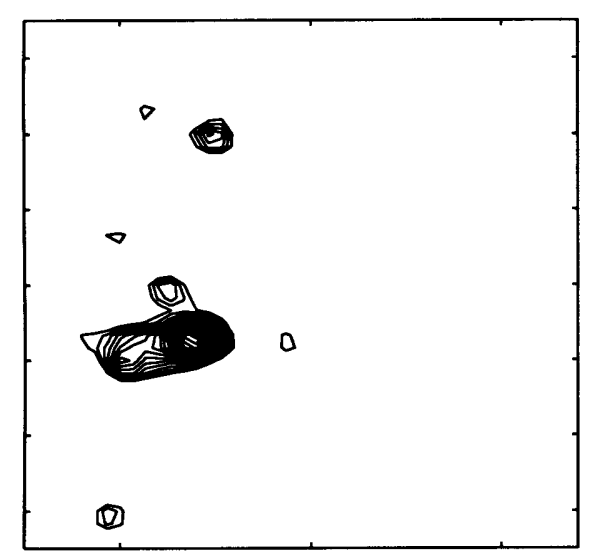

20 sec.

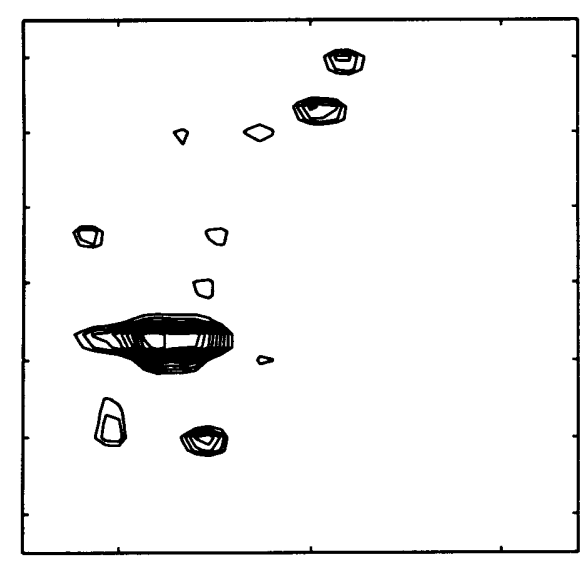

$6 \mathrm{sec}$.

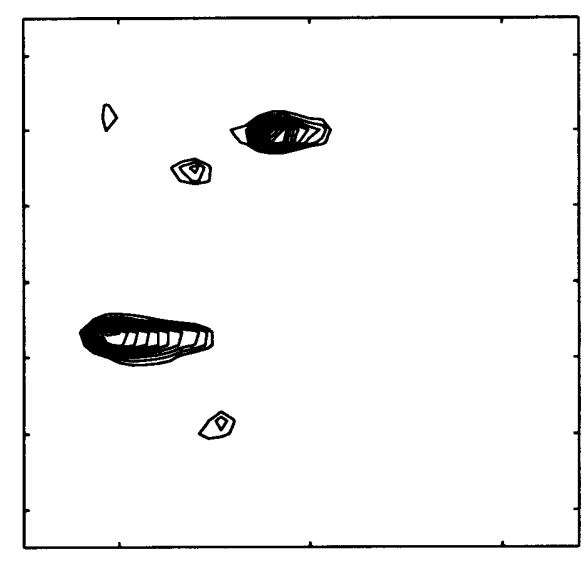

$16 \mathrm{sec}$.

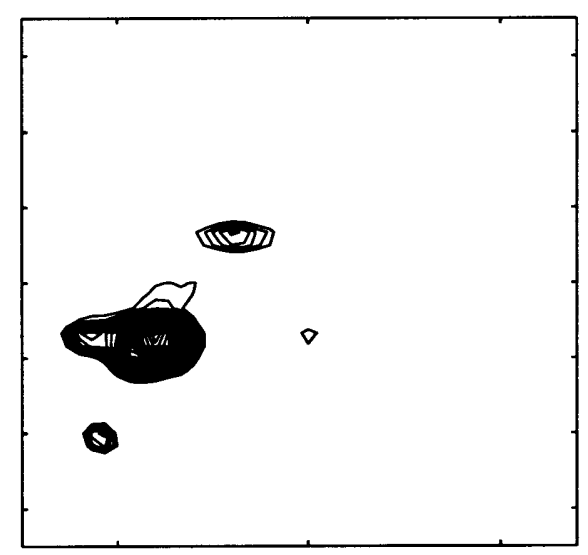

Figure A-10: SET distribution for glaze accretion T184.21.32. 


\section{Appendix B}

\section{Validation of the MRF Code}

Examples of MRF textures produced by Cross and Jain are shown in Figure B-1 along with the clustering parameters used. To minimize the number of calculations, the images have only $1-b i t$ gray-scale (black and white). A typical plot of pixel interchanges per 256 attempted exchanges during the Monte Carlo simulations of Cross and Jain is shown in Figure B-2. As can be seen, there is initially a rapid decrease in the number of pixel interchanges. This trend levels out so that for $M$ attempted interchanges, there are approximately $M / 2$ actual pixel exchanges. Cross and Jain [2] declared this situation to be an equilibrium state, but, as will be discussed later, the MRF texture is actually not in equilibrium.

Textures for identical clustering parameters were generated using the code developed for this study and are shown in Figure B-3. As is evident, these textures match very well with the corresponding textures of Figure B-1. Figure B-4 shows a typical plot of number of interchanges per 256 attempted interchanges. Again, the results compare very well with the work of Cross and Jain [2].

Figure B-5 gives an example of what types of images can be created from a white noise initial condition using additional gray levels and a higher order model. The particular image shown in Figure B-5 also has isotropic clustering parameters. Such images take longer to come to the type of equilibrium described in Cross and Jain [2] and shown in Figure B-2. 


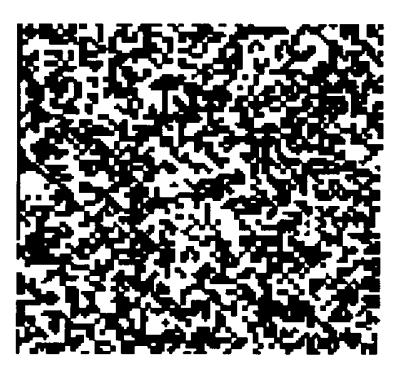

(a) $b(1, \cdot)=0.0$

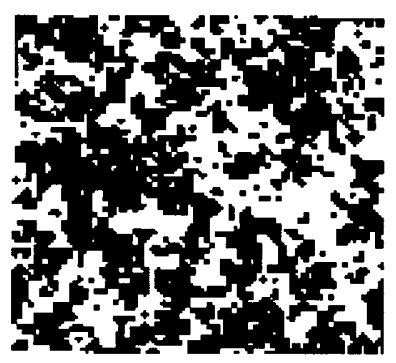

(e) $b(1, \cdot)=1.26$

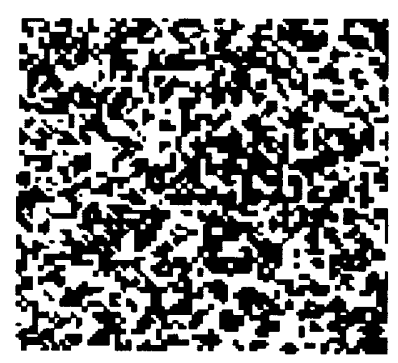

(b) $b(1, \cdot)=0.50$

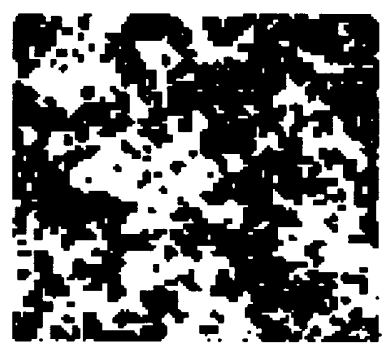

(f) $b(1, \cdot)=1.52$

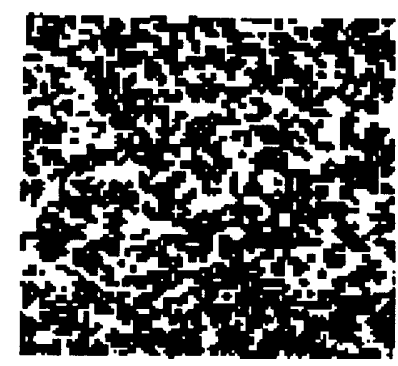

(c) $b(1, \cdot)=0.75$

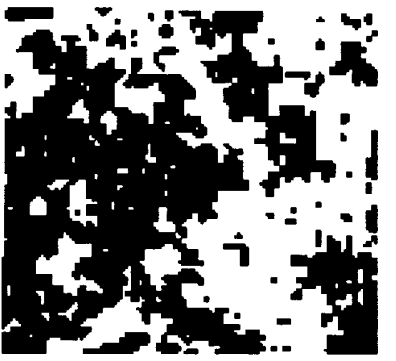

(g) $b(1, \cdot)=1.79$

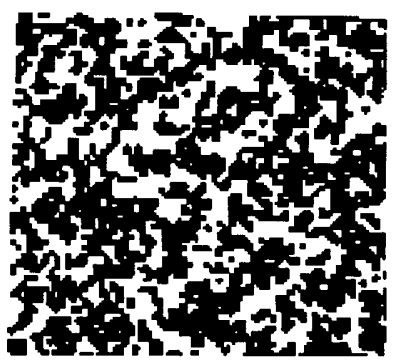

(d) $b(1, \cdot)=1.1$

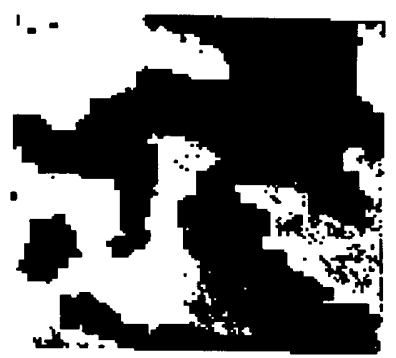

(h) $b(1, \cdot)=3.0$

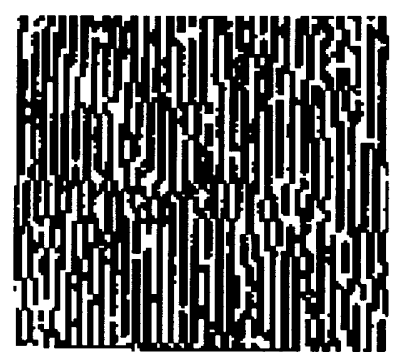

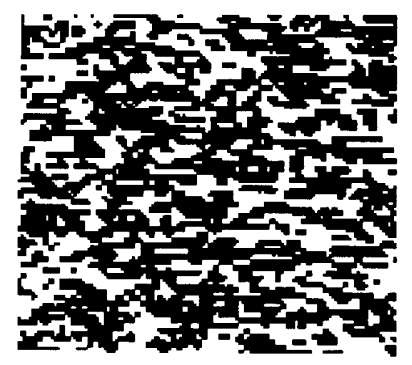

(j) $a=-2.04$

$b(1,1)=1.93$,

$b(1,2)=0.16$,

$b(2,1)=0.07$

$b(2,2)=0.02$

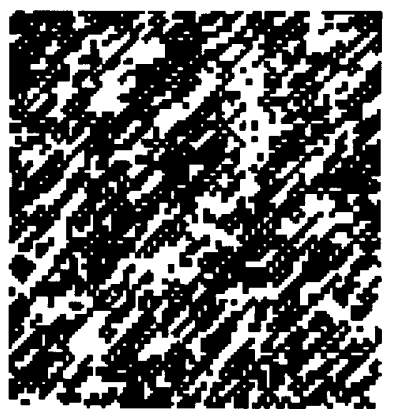

(k) $a=-1.9$,

$b(1,1)=-0.1$,

$b(1,2)=0.1$,

$b(2,1)=-0.075$,

$b(2,2)=1.9$

Figure B-1: MRF Textures of Cross and Jain [2] 


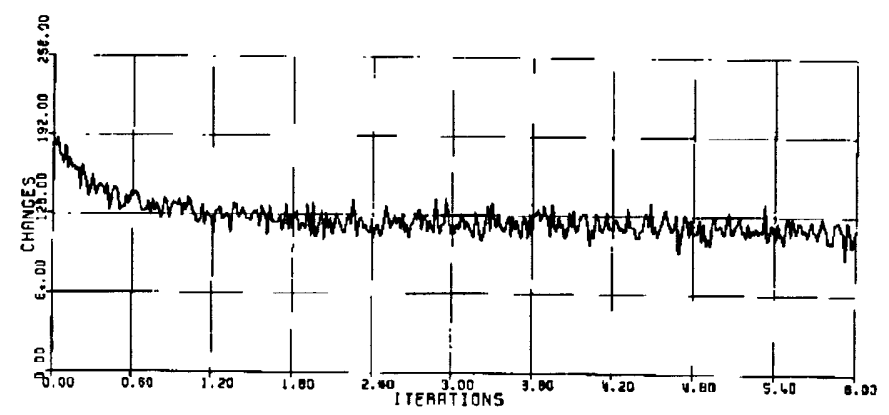

Figure B-2: Typical behavior of number of pixel exchanges for code of Cross and Jain [2]. 1 iteration $=256$ attempted exchanges. 


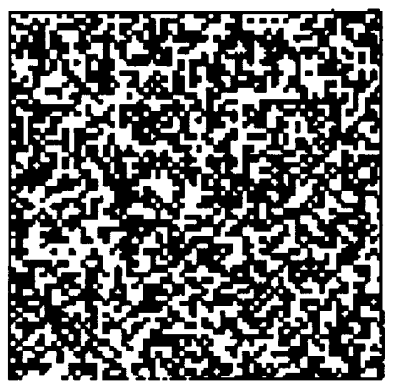

(a) $b(1, \cdot)=0.0$

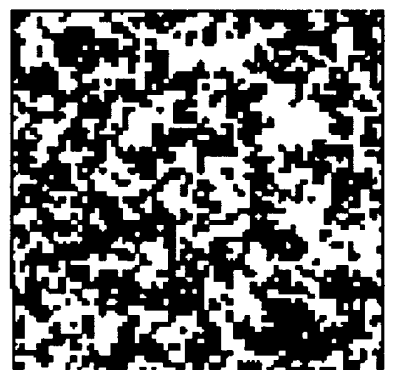

(e) $b(1, \cdot)=1.26$

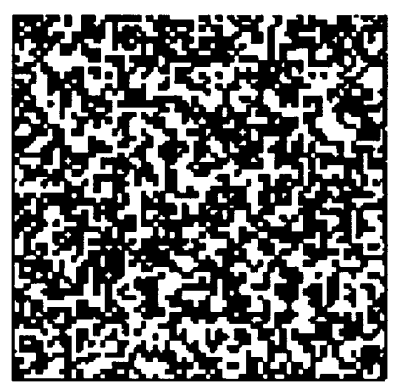

(b) $b(1, \cdot)=0.50$

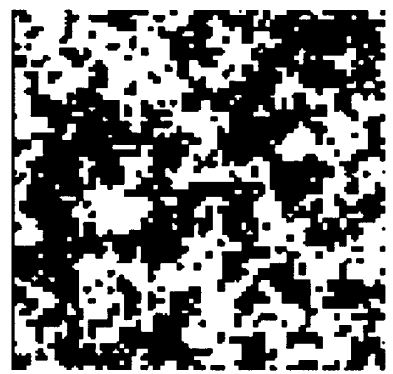

(f) $b(1, \cdot)=1.52$

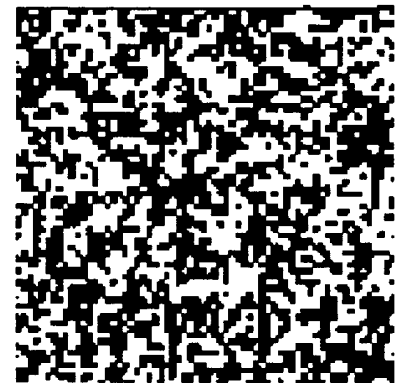

(c) $b(1, \cdot)=0.75$

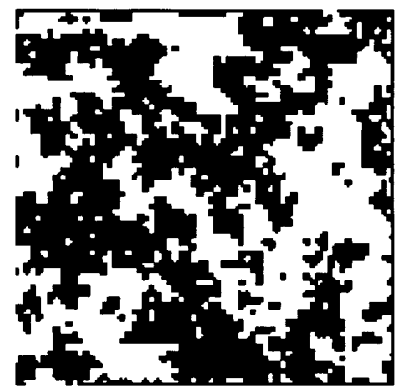

(g) $b(1, \cdot)=1.79$

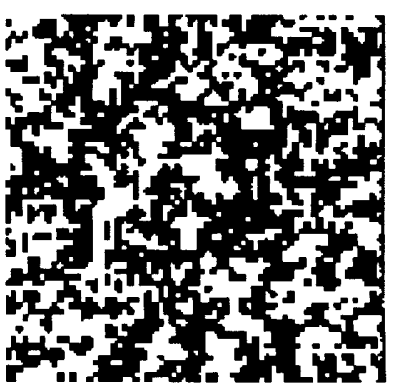

(d) $b(1, \cdot)=1.1$

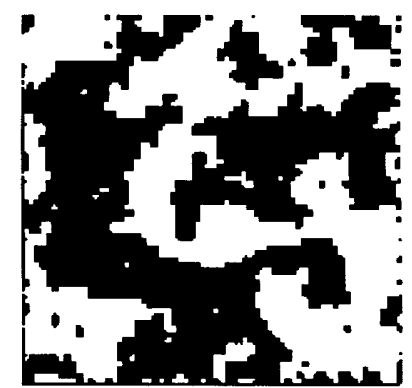

(h) $b(1, \cdot)=3.0$

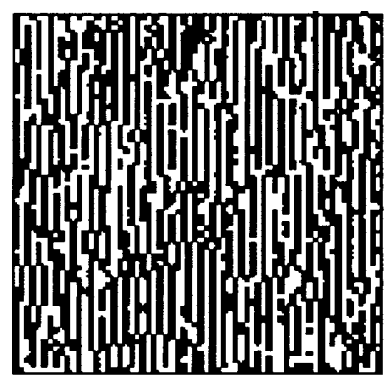

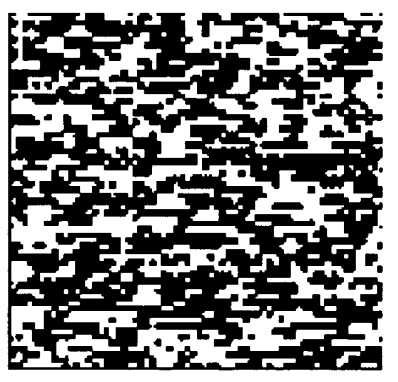

(j) $a=-2.04$,

$b(1,1)=1.93$,

$b(1,2)=0.16$,

$b(2,1)=0.07$,

$b(2,2)=0.02$

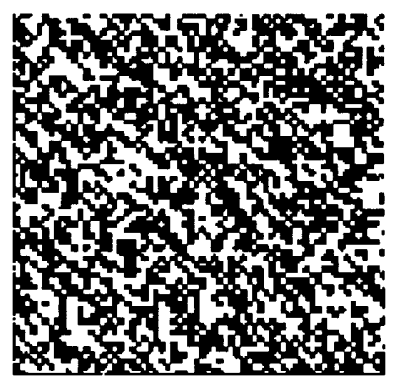

(k) $a=-1.9$,

$b(1,1)=-0.1$,

$b(1,2)=0.1$,

$b(2,1)=-0.075$,

$b(2,2)=1.9$

Figure B-3: MRF Texture Simulation 


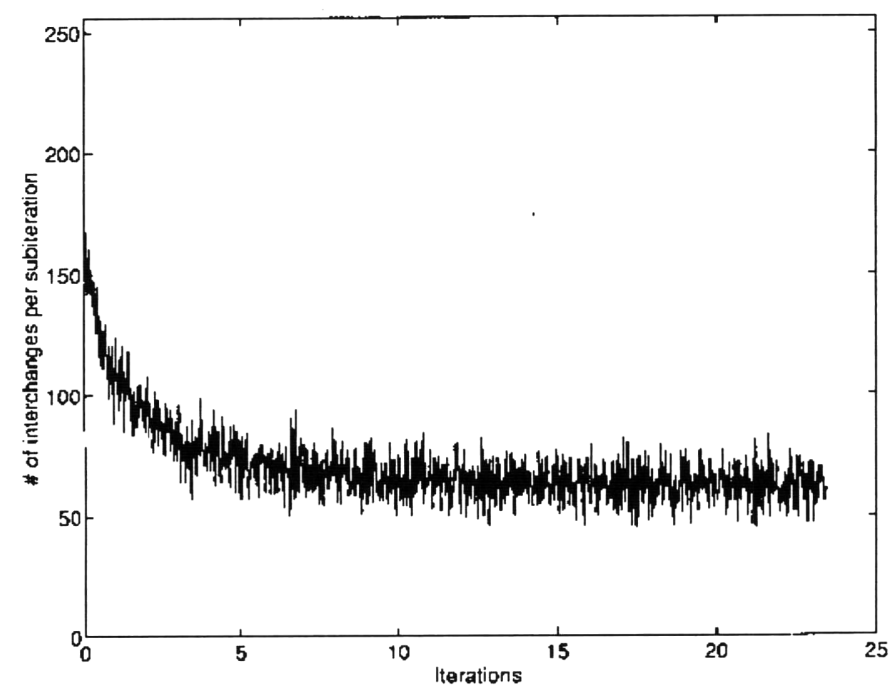

Figure B-4: Behavior of number of pixel exchanges for MRF code of the current study.

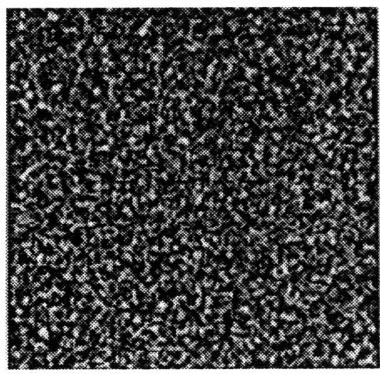

(a) White noise initial condition for 16 gray levels $(128 \times$ 128pixels).

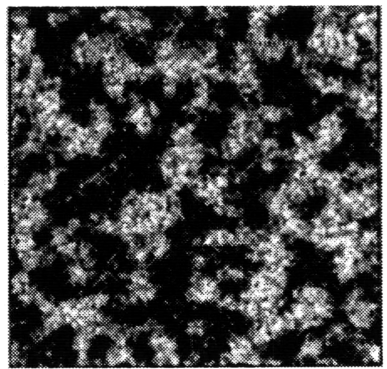

(b) MRF texture image: $4 t h$ order model, 16 gray levels, $\quad a=-2.0$, $b(.,)=$.

Figure B-5: 4 - bit, $4^{\text {th }}$-order, Gray-scale MRF Image 


\section{Appendix $\mathbf{C}$}

\section{Derivations for Markov Random Field Analysis}

\section{C.1 The Method of Rejection/Selection}

Let $X$ be the accepted value obtained in Step 2 above after $N$ iterations. Since $X=Y_{N}$ on the $N^{t h}$ try, by the definition of conditional probability, we have:

$$
p(X \leq x)=p\left(Y_{N} \leq x\right)=p\left(Y \leq x \mid U \leq \frac{f(Y)}{c g(X)}\right)=\frac{p\left(Y \leq x, U \leq \frac{f(Y)}{c g(X)}\right)}{p\left(U \leq \frac{f(Y)}{c g(Y)}\right)}
$$

The joint density function of $Y$ and $U$ is, by independence,

$$
f(y, u)=g(y), 0<u<1 \text {. }
$$

By the definition of a continuous joint probability density function and Equation C.1, we have:

$$
p(X \leq x)=\frac{\int_{0}^{x} \int_{0}^{f(y) / c g(y)} g(y) d u d y}{p\left(U \leq \frac{f(Y)}{c g(Y)}\right)} .
$$

Simplifying this last expression, we have:

$$
p(X \leq x)=\frac{\int_{0}^{x} \frac{f(y)}{c g(y)} g(y) d y}{p\left(U \leq \frac{f(Y)}{c g(Y)}\right)}=\frac{\frac{1}{c} \int_{0}^{x} f(y) d y}{p\left(U \leq \frac{f(Y)}{c g(Y)}\right)}
$$

Since $f$ is a probability density function, we observe that

$$
\lim _{x \rightarrow \infty} p(X \leq x)=\frac{\int_{0}^{\infty} f(y) d y}{c p\left(U \leq \frac{f(Y)}{c g(Y)}\right)}=\frac{1}{c p\left(U \leq \frac{f(Y)}{c g(Y)}\right)}=1 .
$$

This implies

$$
p\left(U \leq \frac{f(Y)}{c g(Y)}\right)=\frac{1}{c}
$$


Combining Equations C.3 and C.2, we see that

$$
p(X \leq x)=c \int_{0}^{x} \frac{f(y)}{c g(y)} g(y) d y=\int_{0}^{x} f(y) d y
$$

This shows that our simulation of $X$ has lead to the simulation of the desired random variable, $Y$. This scheme is known as the method of rejection/selection.

\section{C.2 Besag's Lattice Probability Quotient}

By the definition of conditional probability, we may write:

$$
p(\mathbf{X})=p\left(x_{M} \mid x_{1}, x_{2}, \ldots, x_{M-1}\right) p\left(x_{1}, x_{2}, \ldots, x_{M-1}\right) \text {. }
$$

For our lattice with $M$ sites, we can compute the above conditional probability for any lattice site. However, it is not so easy to compute from a reduced set of random variables:

$p\left(x_{1}, \ldots, x_{M-1}\right)=p\left(x_{k} \mid x_{1}, \ldots, x_{k-1}, x_{k+1}, \ldots, x_{M-1}\right) p\left(x_{1}, \ldots, x_{k-1}, x_{k+1}, \ldots, x_{M-1}\right)$.

Since each of the lattice random variables can range over $G$ gray levels, introduce $y_{M}$, an alternative gray level that the random variable $X_{M}$ can assume other than $x_{M}$. Note that

$$
p\left(x_{1}, \ldots, x_{M-1}\right)=\frac{p\left(x_{1}, \ldots, x_{M-2}, x_{M-1}, y_{M}\right)}{p\left(y_{M} \mid x_{1}, \ldots, x_{M-1}\right)} .
$$

Combining Equations C.5 and C.6 yields:

$$
p(\mathbf{X})=p\left(x_{1}, \ldots, x_{M}\right)=p\left(x_{M} \mid x_{1}, \ldots, x_{M-1}\right) \frac{p\left(x_{1}, \ldots, x_{M-1}, y_{M}\right)}{p\left(y_{M} \mid x_{1}, \ldots, x_{M-1}\right)} .
$$

But

$$
p\left(x_{1}, \ldots, x_{M-1}, y_{M}\right)=p\left(x_{M-1} \mid x_{1}, \ldots, x_{M-2}, y_{M}\right) p\left(x_{1}, \ldots, x_{M-2}, y_{M}\right)
$$

and

$$
p\left(x_{1}, \ldots, x_{M-2}, y_{M}\right)=\frac{p\left(x_{1}, \ldots, x_{M-2}, y_{M-1}, y_{M}\right)}{p\left(y_{M-1} \mid x_{1}, \ldots, x_{M-2}, y_{M}\right)} .
$$

Combining Equations C.8 and C.9 yields:

$$
p\left(x_{1}, \ldots, x_{M-1}, y_{M}\right)=p\left(x_{M-1} \mid x_{1}, \ldots, x_{M-2}, y_{M}\right) \frac{p\left(x_{1}, \ldots, x_{M-2}, y_{M-1}, y_{M}\right)}{p\left(y_{M-1} \mid x_{1}, \ldots, x_{M-2}, y_{M}\right)}
$$


Combining Equations C.7 and C.10 produces:

$$
p(\mathbf{X})=\frac{p\left(x_{M} \mid x_{1}, \ldots, x_{M-1}\right) p\left(x_{M-1} \mid x_{1}, x_{M-2}, y_{M}\right)}{p\left(y_{M} \mid x_{1}, \ldots, x_{M-1}\right) p\left(y_{M-1} \mid x_{1}, \ldots, x_{M-2}, y_{M}\right)} p\left(x_{1}, \ldots, x_{M-2}, y_{M-1}, y_{M}\right)
$$

But,

$p\left(x_{1}, \ldots, x_{M-2}, y_{M-1}, y_{M}\right)=p\left(x_{M-2} \mid x_{1}, \ldots, x_{M-3}, y_{M-1}, y_{M}\right) p\left(x_{1}, \ldots, x_{M-3}, y_{M-1}, y_{M}\right)$.

And

$$
p\left(x_{1}, \ldots, x_{M-3}, y_{M-1}, y_{M}\right)=\frac{p\left(x_{1}, \ldots, x_{M-3}, y_{M-2}, y_{M-1}, y_{M}\right)}{p\left(y_{M-2} \mid x_{1}, \ldots, x_{M-3}, y_{M-1}, y_{M}\right)}
$$

Combining Equations C.12 and C.13 produces:

$p\left(x_{1}, \ldots, x_{M-2}, y_{M-1}, y_{M}\right)=p\left(x_{M-2} \mid x_{1}, \ldots, x_{M-3}, y_{M-1}, y_{M}\right) \frac{p\left(x_{1}, \ldots, x_{M-3}, y_{M-2}, y_{M-1}, y_{M}\right)}{p\left(y_{M-2} \mid x_{1}, \ldots, x_{M-3}, y_{M-1}, y_{M}\right)}$.

Combining Equations C.14 and C.11 yields:

$$
\begin{gathered}
p(\mathbf{X})=\frac{p\left(x_{M} \mid x_{1}, \ldots, x_{M-1}\right) p\left(x_{M-1} \mid x_{1}, \ldots, x_{M-2}, y_{M}\right) p\left(x_{M-2} \mid x_{1}, \ldots, x_{M-3}, y_{M-1}, y_{M}\right)}{p\left(y_{M} \mid x_{1}, \ldots, x_{M-1}\right) p\left(y_{M-1} \mid x_{1}, \ldots, x_{M-2}, y_{M}\right) p\left(y_{M-2} \mid x_{1}, \ldots, x_{M-3}, y_{M-1}, y_{M}\right)} \\
\times p\left(x_{1}, \ldots, x_{M-3}, y_{M-2}, y_{M-1}, y_{M}\right)
\end{gathered}
$$

Repeating this process $M-3$ more times produces the desired formula:

$$
\frac{p(\mathbf{X})}{p(\mathbf{Y})}=\prod_{i=1}^{M} \frac{p\left(x_{i} \mid x_{1}, \ldots, x_{i-1}, y_{i+1}, \ldots, y_{M}\right)}{p\left(y_{i} \mid x_{1}, \ldots, x_{i-1}, y_{i+1}, \ldots, y_{M}\right)}
$$




\section{Bibliography}

[1] Julian Besag. Spatial interaction and the statistical analysis of lattice systems. Journal of the Royal Statistical Society, 36(2):192-326, March 1974.

[2] George R. Cross and Anil K. Jain. Markov random field texture models. IEEE Transactions on Pattern Analysis and Machine Intelligence, 5(1):25-39, January 1983.

[3] Louis Garand and James A. Weinman. A structural-stochastic model for the analysis and synthesis of cloud images. Journal of Climate and Applied Meteorology, 25(1):1052-1068, July 1986.

[4] R. C. Henry, K. S. Breuer, and R. J. Hansman Jr. Measurement of heat transfer variation on surface roughness elements using infrared techniques. Journal of Thermophysics and Heat Transfer, 1994.

[5] R. J. Hansman Jr., K. S. Breuer, D. Hazan, A. Reehorst, and M. Vargas. Closeup analysis of aircraft ice accretion. AIAA Paper 93-0029, January 1993.

[6] M. F. Kerho and M. B. Bragg. Effect of large distributed leading-edge roughness on boundary layer development and transition. AIAA Paper 95-1803, January 1995.

[7] Christine K. Lindsay. Implementation of a two-dimensional Markov random field texture model. MIT Undergraduate Thesis, May 1988.

[8] K. C. Masiulaniec, K. J. DeWitt, and N. Dukhan. Experimental technique and assessment of measuring the convective heat transfer from natural ice accretions. AIAA Paper 95-0537, January 1995.

[9] N. Metropolis, A. W. Rosenbluth, M. N. Rosenbluth, A. H. Teller, and E. Teller. Equations of state calculations by fast computing machines. Journal of Chemical Physics, 21(1):1087-1091, January 1953.

[10] J. Nikuradse. Laws of flow in rough pipes. NACA TM-1292, 1933.

[11] W. Olsen and E. Walker. Experimental evidence for modifying the current physical model for ice accretion on aircraft surfaces. NASA TM-87184, 1986. 
[12] D. J. Orr, K. S. Breuer, and R. J. Hansman Jr. Quantitative analysis of ice accretion roughness using spectral and stochastic techniques. AIAA Paper 950888, January 1995.

[13] P. Personne and C. Duroure. Influence de la rugosite du givre sur sa croissance par la captation des gouttes d'eau surfondue: application au givrage des cables electriques. Journal de Physique, 48:389-395, 1987.

[14] R. W. Picard. Texture Modeling: Temperature Effects on Markov/Gibbs Random Fields. PhD thesis, Massachusetts Institute of Technology, 1991.

[15] William H. Press, Saul A. Teukolsky, William T. Vetterling, and Brian P. Flannery. Numerical Recipes in C. Cambridge University Press, New York, New York, second edition, 1992.

[16] Sheldon Ross. A First Course in Probability. Macmillan Publishing Company, New York, New York, third edition, 1988.

[17] H. Schlichting. Experimental investigation of the problem of surface roughness. NACA TM-823, 1937.

[18] J. W. Smith and N. Epstein. Effect of wall roughness on convective heat transfer in commercial pipes. A.I.Ch.E., pages 242-248, 1957.

[19] B. E. Torres. Heat transfer measurements on surfaces with ice accretion roughness. Master's thesis, Massachusetts Institute of Technology, 1996.

[20] K. Yamaguchi and R. J. Hansman Jr. Heat transfer on accreting ice surfaces. AIAA Paper 90-0200, January 1990.

[21] K. Yamaguchi, R. J. Hansman Jr., and M. Kazmierczak. Deterministic multizone ice accretion modeling. AIAA Paper 91-0265, January 1991. 